\title{
Methodology for the 2005 USGS Assessment of Undiscovered Oil and Gas Resources, Central North Slope, Alaska
}

By John H. Schuenemeyer ${ }^{1}$

${ }^{1}$ Southwest Statistical Consulting, LLC, 960 Sligo St., Cortez, CO 81321

Open-File Report 2005-1410 


\section{U.S. Department of the Interior \\ Gale A. Norton, Secretary \\ U.S. Geological Survey \\ P. Patrick Leahy, Acting Director}

U.S. Geological Survey, Menlo Park, California: 2005

For more information about the USGS and its products:

Telephone: 1-888-ASK-USGS

World Wide Web: http://www.usgs.gov/

Any use of trade, product, or firm names in this publication is for descriptive purposes only and does not imply endorsement by the U.S. Government.

Although this report is in the public domain, permission must be secured from the individual copyright owners to reproduce any copyrighted materials contained within this report. 


\section{Contents}

Abstract

Introduction

Specification of the Input

Minimum accumulation size (MAS)

Oil accumulation attributes

Oil accumulation characteristics and estimate of in-place oil

Gas accumulation attributes, characteristics and estimate of in place gas

Derivatives

Correlated attributes

Risking

Graphical feedback

The deposit simulation

Aggregation methodology

Overview

Specifying the dependency

Generating a correlated sample

References

Appendix A. Brief description of Splus functions used

Appendix B. Instructions to assessors concerning the protocol

Appendix C. Glossary

Appendix D. Algorithms

\section{Figures}

1. Oil formation volume factor vs. trap depth for selected oil gravities.

2. Gas compressibility factor vs. trap depth.

3. Gas formation volume factor vs. trap depth.

4. General flow chart for simulation algorithm.

\section{Tables}

1a. Oil attribute input sheet.

1b. Oil correlation attributes and engineering computations.

1c. Number of prospects distributions.

1d. Prospect and play risking.

1e. Nonassociated gas input sheet.

1f. Nonassociated gas correlation attributes and engineering computations.

$1 \mathrm{~g}$. Shapes to be used to specify accumulation attributes, trap depth, and number of prospects.

2a. Instantaneous feedback graphs, Beaufortian Clinoform play.

2b. Model fitted beta distributions to oil and nonassociated gas attributes, Beaufortian Clinoform play.

2c. Oil accumulation distribution, Beaufortian Clinoform play.

2d. Oil accumulation distribution, Beaufortian Clinoform play, log units.

3. Play number, name, favorability probability (risk), and file name.

4. Sample simulation output from the Beaufortian Clinoform play.

5a. Assessor specified charge dependencies between plays.

5 b. Assessor specified trap dependencies between plays.

5c. Assessor specified timing dependencies between plays.

$5 \mathrm{~d}$. Arithmetic average of assessor specified Charge, Trap, and Timing correlations.

5 e. Adjusted dependency matrix.

6. Partial aggregation file.

7. Summary statistics for distributions of technically recoverable undiscovered oil, nonassociated gas and derivatives for the central North Slope assessment area. 


\title{
METHODOLOGY FOR THE 2005 USGS ASSESSMENT OF OIL AND GAS RESOURCES, CENTRAL NORTH SLOPE, ALASKA
}

\author{
By John H. Schuenemeyer
}

\begin{abstract}
Undiscovered oil and gas resources in each of 24 plays (assessment units) within the Alaska Central North Slope were estimated using a deposit simulation analysis in the assessment by the U.S. Geological Survey in 2005. Plays were identified by assessor geologists who specified geologic attributes, risks, and number of prospects for each play. From this information, sizes of oil and gas accumulations were simulated using a Monte Carlo algorithm. The number of such accumulations considered in a given simulation run was obtained from the distribution of the number of prospects. Each prospect in each successful simulation run was risked. This process yielded sizefrequency distributions and summary statistics for the various petroleum categories. Estimates of resources from individual plays were then aggregated, and measures of uncertainty computed.
\end{abstract}

\section{Introduction}

The 2005 USGS Central North Slope assessment was performed at the play level (Bird and others, 2005; Garrity and others, 2005). For each play, assessors specified distributions needed to generate accumulations of oil and gas, and distributions of the number of prospects expected to occur. They also specified risk and recovery factors. The methodology used in this assessment was a modified version of the methodology (Schuenemeyer, 2003) used in the U.S. Geological Survey's 2002 assessment of the National Petroleum Reserve Alaska (NPRA) (Bird and Houseknecht, 2002). Improvements included separate specification of numbers of oil and gas prospects, modifications to the input form, expanded graphics, and updated petroleum engineering models. Minimum reservoir sizes for oil and for gas were established to facilitate estimation of the number of prospects. Approximate deposit size distributions were generated at the mean, median, and $5^{\text {th }}$ and $95^{\text {th }}$ levels of uncertainty. The assessment process was based on expert judgment. The final play results were developed by consensus. A detailed discussion of the results is presented in Garrity and others (2005).

This report begins with a discussion of the geologic and engineering input, which was specified by the assessors for each play and entered on assessment forms. Following a discussion of input data and construction of oil and gas accumulation distributions, the Monte Carlo simulation is presented. We conclude with a discussion of the aggregation procedure. 


\section{Specification of the input}

Information used by the assessment algorithm consisted of statistical models with parameters and assessor-specified distributions and constants. An assessment form, which was a modified version of that used in the 2002 USGS NPRA assessment, was used to capture the distributions and constants for each play. The input for oil, gas, and number of prospects and risk was specified on Excel ${ }^{1}$ worksheets. We illustrate input attributes with the Beaufortian Clinoform play forms (table 1a through table 1g). Note that the information on these forms is for illustration only and may not correspond to the final play input. The first worksheet (table 1a) provided for the entry of accumulation attributes for oil, the trap depth distribution, and oil accumulation characteristics. Table $1 \mathrm{~b}$ displayed a form to specify pairwise correlations between accumulation attributes. It also displayed engineering computations - the gas to oil ratio $(G O R)$, and a graph (not shown) of the formation volume factor for oil $\left(F V F_{\mathrm{o}}\right)$ versus trap depth. In Table 1c the number of prospects was specified for technically recoverable accumulations of at least 5 $\mathrm{mm}$ bbl for oil and $100 \mathrm{bcf}$ for gas. Table $1 \mathrm{~d}$ was used to specify play and prospect risk. Separate prospect risks were given for oil and for gas. Table 1e provided for nonassociated gas volume attributes and gas characteristic values. Table $1 \mathrm{f}$ displayed a form to specify pairwise correlations between accumulation attributes for nonassociated gas. It also displayed a graph (not shown) of the formation volume factor for oil $\left(F V F_{\mathrm{g}}\right)$ versus trap depth.

In addition to specifying fractiles of a distribution, assessors were asked to specify its shape, which could range from highly right skewed to highly left skewed (table $1 \mathrm{~g}$ ). This procedure served as a check on the specification of fractiles and as initial values for the distribution fitting algorithm. General instructions for completing these forms were provide to each assessor in the protocol (Appendix B).

\section{Minimum accumulation size (MAS)}

To avoid the considerable uncertainties associated with assessing a potentially large number of small prospects, which would be neither technically recoverable nor commercially viable in the foreseeable future, the assessment team established a minimum reservoir size or cutoff value for oil and gas. For oil, only deposits of at least 5 $\mathrm{mm}$ bbl technically recoverable were considered. For gas, only deposits of at least 100 bcf technically recoverable were considered. These choices of MAS allow for fields, which are now marginally economic, to be considered if the price/cost ratio becomes more favorable.

\footnotetext{
${ }^{1}$ Mention of a brand name is provided for clarification and does not constitute an endorsement by the U.S. Geological Survey or the author.
} 
Table 1a. Oil attribute input sheet.

\begin{tabular}{|c|c|}
\hline Alaskan Assess & sment Form \\
\hline PLAY: & Beaufortian Clinoform \\
\hline Oil Play Area: & $1,447 \mid$ thousands of acres \\
\hline
\end{tabular}

OIL ACCUMULATION ATTRIBUTE DISTRIBUTIONS

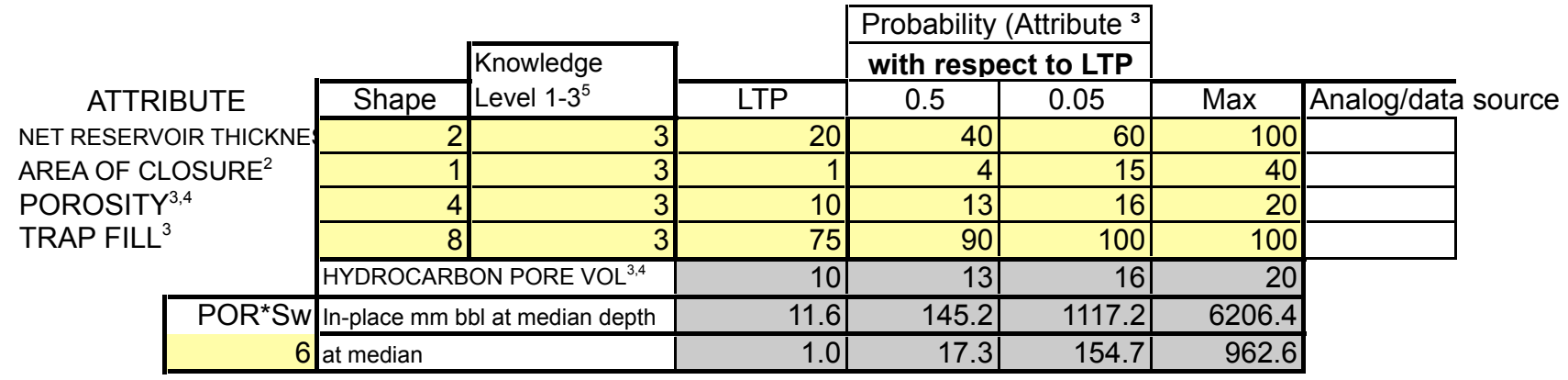

1-thickness in feet, 2-thousands of acres, 3-percent, 4-correlation between Porosity and Water Saturation $=-1.0$ 5-Knowledge Level: 1=High, 2=Medium, 3=Low; LTP=Lower Truncation Point

\begin{tabular}{|l|l|l|l|r|r|r|}
\multicolumn{1}{l|}{} & Shape & Level $1-3^{5}$ & Min & 0.5 & 0.05 & \multicolumn{1}{c|}{ Max } \\
\hline $\begin{array}{l}\text { TRAP DEPTH (in 1000 ft) } \\
\text { (from sea level) }\end{array}$
\end{tabular}

\section{OIL ACCUMULATION CHARACTERISTICS}

Oil recovery factor $\%$ 40

Type of reservoir-drive (check any that apply): Water: Depletion: $x$

$x$

Gas expansion:

FVF (Formation volume factor, rb/stb):

1.81 (at median depth) (see OilDet sheet)

$F V F=0.972+0.000147^{*} F^{\wedge} 1.175$

NGLR (Natural gas liquids to associated gas ratio, bbls/million cu.ft., at $\operatorname{stp})=4.5985^{\star} \exp \left(.1711^{*} \mathrm{TD}\right.$ )

Oil quality parameters:

API gravity

Oil Grav (ratio)

Sulfur content of oil

Associated gas quality parameters:

Hydrogen sulfide \%

CO2 contamination \%

Other inert gases:

Name:

Name:

25.5 (at median depth)

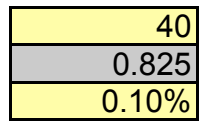

$0.10 \%$
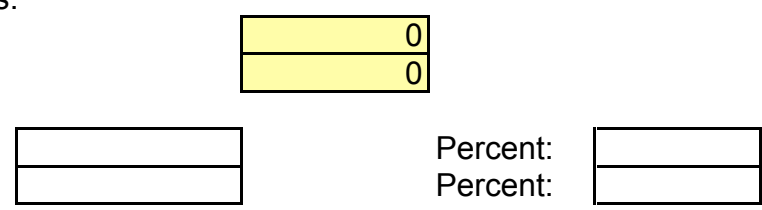

TIME OF TRAP DEVELOPMENT

STRATIGRAPHIC COMPONENT (Ma)

STRUCTURAL COMPONENT (Ma)

\begin{tabular}{|r|r|r|}
\multicolumn{1}{l|}{ BEGIN } & PEAK & \multicolumn{1}{c}{ END } \\
\hline 159 & 135 & 130 \\
\hline 100 & 65 & 25 \\
\hline
\end{tabular}

Assessor's Name: DWH

Date of Data Entry MM/DD/YYYY:

Date of Simulation Run MM/DD/YYYY: 
Table 1b. Oil correlation attributes and engineering computations

\section{Alaskian Assessment Form $\quad$ Rev: 14-Oct-04 \\ PLAY: Beaufortian Clinoform}

\section{OIL ACCUMULATION ATTRIBUTE DEPENDENCIES}

\begin{tabular}{|c|c|c|c|c|}
\hline Correlations & $\begin{array}{l}\text { NET } \\
\text { RESERVOIR } \\
\text { THICKNESS }\end{array}$ & $\begin{array}{l}\text { AREA OF } \\
\text { CLOSURE }\end{array}$ & TRAP FILL & $\begin{array}{l}\text { HYDROCARBO } \\
\text { N PORE VOL }\end{array}$ \\
\hline NET RESERVOIR THICKNESS & \begin{tabular}{r|}
1 \\
\end{tabular} & $\begin{array}{r}0.0 \\
\end{array}$ & 0.0 & $\begin{array}{r}0.0 \\
\end{array}$ \\
\hline AREA OF CLOSURE & 0.0 & 1 & 0.0 & 0.0 \\
\hline TRAP FILL & 0.0 & 0.0 & 1 & 0.0 \\
\hline HYDROCARBON PORE VOL & 0.0 & 0 & 0 & 1 \\
\hline
\end{tabular}

FVF (Formation volume factor, rb/stb):

\begin{tabular}{|c|c|c|}
\hline Median Trap Dep & 10 & \\
\hline \multirow{6}{*}{$\begin{array}{l}\text { Pressure, psi (P) } \\
\text { Temp, deg F (T) } \\
\text { Soution Gas Gr (SGG) } \\
\text { Uncorrected GOR } \\
\text { Final GOR at median depth } \\
\text { F }\end{array}$} & 5000 & Pressure $=$ TrapDepth(thous $\mathrm{ft})^{*} 0.5^{*} 1000$ \\
\hline & 220 & Temp $=19^{*}$ TrapDepth +30 \\
\hline & 0.767 & $S G G=\left(\left(0.1402^{*} \operatorname{LN}(P+14.7)-0.4227\right)+\left(0.1369^{*} \mathrm{LN}(\mathrm{T})+0.0156\right)+\left(0.1704^{*} \mathrm{LN}(\right.\right.$ API_gravity $\left.\left.)+0.1469\right)\right) / 3$ \\
\hline & 1555 & Uncorrected GOR $=\mathrm{SGG}^{*}\left((\mathrm{P}+14.7)^{\star} 10^{\wedge}\left(0.0125^{\star} \mathrm{API} \text { gravity }\right) /\left(18^{*} 10^{\wedge}\left(0.00091^{*} \mathrm{~T}\right)\right)\right)^{\wedge}(1 / 0.83)$ \\
\hline & 1338 & Approx final GOR If(Uncorrected GOR $<=1800,0.86^{*}$ Un GOR, Un GOR) \\
\hline & 1565 & $\mathrm{~F}=$ Final $\mathrm{GOR}^{\star}(\mathrm{SGG} / \mathrm{OG})^{\wedge} 0.5+1.25^{\star} \mathrm{T}$ \\
\hline & s: & $\begin{array}{l}\text { at median depth is associated gas to oil ratio, cu.ft./bbl, at stp } \\
\text { ase e }\end{array}$ \\
\hline
\end{tabular}

Assessor's Name:

Date of Data Entry MM/DD/YYYY:

\begin{tabular}{|l|r|}
\hline DWH \\
\hline \multirow{2}{*}{$10 / 15 / 04$} \\
\hline
\end{tabular}

Date of Simulation Run MM/DD/YYYY: 
Table 1c. Number of prospects distributions

Alaskan Assessment Form

\begin{tabular}{|l|c|}
\hline PLAY: & Beaufortian Clinoform \\
\hline Oil Play Area: & $\mathbf{1 , 4 4 7}$ thousands of acres \\
Gas Play Area: & $\mathbf{1 , 6 3 2}$ thousands of acres \\
\hline
\end{tabular}

Rev: 14-Oct-04

\section{OIL PROSPECTS}

MINIMUM ACCUMULATION SIZE, MAS

5 $\mathrm{mm}$ bbl, recoverable

\begin{tabular}{|c|c|c|c|c|c|c|}
\hline & & \multirow{2}{*}{$\begin{array}{l}\text { Knowledge } \\
\text { Level } 1-3^{5}\end{array}$} & & \multicolumn{2}{|c|}{ Prob (Num. of } & \multirow[b]{2}{*}{ Max } \\
\hline & Shape & & Min & 0.5 & 0.05 & \\
\hline NUM OF PROSPECTS & 2 & 3 & 20 & 40 & 60 & 100 \\
\hline \multicolumn{7}{|c|}{ 5-Knowledge Level: 1=High, 2=Medium, 3=Low } \\
\hline \multirow{2}{*}{\multicolumn{3}{|c|}{$\begin{array}{c}\text { Size thousands acres (median closure): } \\
\text { percent of oil play area }\end{array}$}} & 80 & 160 & 240 & 400 \\
\hline & & & 5.5 & 11.1 & 16.6 & 27.7 \\
\hline
\end{tabular}

\section{NONASSOCIATED GAS PROSPECTS}

MINIMUM ACCUMULATION SIZE, MAS

100 bcf, recoverable

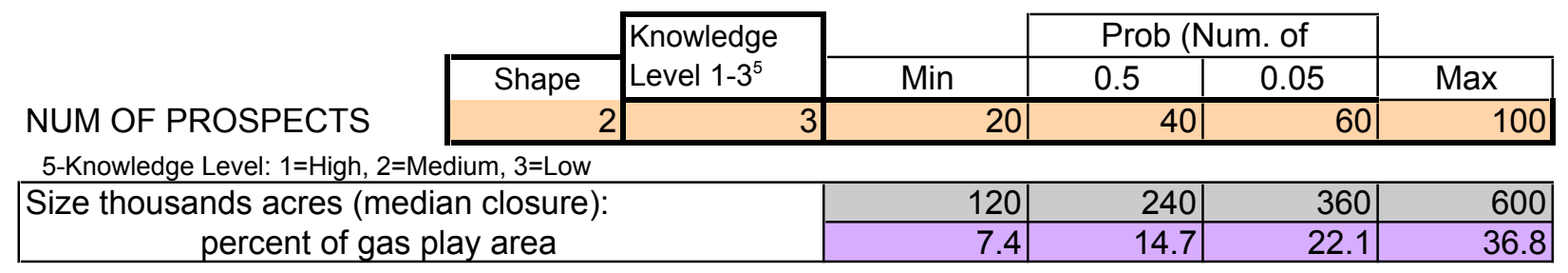

\begin{tabular}{l|r|}
\hline Assessor's Name: $\quad$ DWH \\
Date of Data Entry MM/DD/YYYY: & $9 / 20 / 04$ \\
\hline Date of Simulation Run MM/DD/YYYY: & $10 / 15 / 04$ \\
\hline
\end{tabular}


Table 1d. Prospect and play risking.

Alaskan Assessment Form

Play: Beaufortian Clinoform

\section{PROSPECT \& PLAY RISKING}

\section{ATTRIBUTES OF FAVORABLE}

\begin{tabular}{ll|r|} 
PLAY & Play \\
ATTRIBUTES & ThARGE $(\mathrm{C})$ & 1 \\
& TIMING $(F)$ & 0.6 \\
\hline
\end{tabular}

Probability that play contains at least 1 reservoir $>=$ minimum accumulation size (CxTxF)

Note: Specification of both Oil and Gas play risk attributes implies definition of separate oil \& gas plays

PROSPECT ATTRIBUTES

CHARGE (c) TRAP/ROCK $(\mathrm{t})$ TIMING (f)

Probability that a randomly chosen prospect $>=$ minimum accumulation size is favorable (cxtxf)

Play Attributes x Prospect Attributes (CxTxFxcxtxf)

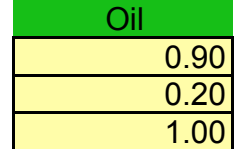

1.00

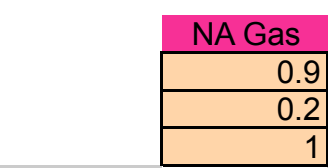

0.18 0.18 0.108

\begin{tabular}{|c|c|c|c|c|}
\hline & Land Area & Oil Vol & Gas Vol \\
\hline \multicolumn{5}{|c|}{ Percent state } \\
\hline \multirow[t]{2}{*}{ State } & Onshore & & & \\
\hline & Offshore & & & \\
\hline \multicolumn{5}{|c|}{ Percent native } \\
\hline \multirow[t]{2}{*}{ Native } & Onshore & & & \\
\hline & Offshore & & & \\
\hline \multicolumn{2}{|c|}{ Total percent } & & & \\
\hline
\end{tabular}

Assessor's Name:

DWH

Date of Data Entry MM/DD/YYYY:

Date of Simulation Run MM/DD/YYYY: 
Table 1e. Nonassociated gas input sheet.

Alaskan Assessment Form
PLAY: Beaufortian Clinoform
Gas Play Area: $\quad 1,632$ thousands of acres

\section{NONASSOCIATED GAS ACCUMULATION ATTRIBUTE DISTRIBUTIONS}

\begin{tabular}{|c|c|c|c|c|c|c|c|}
\hline \multirow{3}{*}{ ATTRIBUTE } & \multirow{3}{*}{ Shape } & \multirow{3}{*}{$\begin{array}{l}\text { Knowledge } \\
\text { Level } 1-3^{5}\end{array}$} & & \multirow{2}{*}{\multicolumn{2}{|c|}{\begin{tabular}{|l|} 
Probability (Attribute $^{3}$ \\
with respect to LTP
\end{tabular}}} & \multirow[b]{3}{*}{ Max } & \multirow[b]{3}{*}{ Analog/data source } \\
\hline & & & & & & & \\
\hline & & & LTP & 0.5 & 0.05 & & \\
\hline \multirow{5}{*}{$\begin{array}{l}\text { NET RESERVOIR THICKNE } \\
\text { AREA OF CLOSURE } \\
\text { POROSITY' } \\
\text { TRAP FILL }^{3}\end{array}$} & 2 & 3 & 30 & 50 & 80 & 100 & \\
\hline & 2 & 3 & 3 & 6 & 15 & 40 & \\
\hline & 4 & 3 & 10 & 12 & 14 & 18 & \\
\hline & 8 & 3 & 75 & 90 & 100 & 100 & \\
\hline & \multicolumn{2}{|c|}{ HYDROCARBON PORE VOL ${ }^{3,4}$} & 10 & 12 & 14 & 18 & \\
\hline $\mathrm{POR}^{*} \mathrm{Sw}$ & \multicolumn{2}{|c|}{ In-place bcf } & 0.29 & 1.41 & 7.32 & 31.36 & \\
\hline & \multicolumn{2}{|c|}{$6 \longdiv { \text { depth } }$} & 0.0 & 0.0 & 0.0 & 0.0 & \\
\hline & \multicolumn{2}{|l|}{ depth } & 0.0 & 0.0 & 0.0 & 0.0 & \\
\hline & \multicolumn{2}{|l|}{ depth } & 0.0 & 0.0 & 0.0 & 0.0 & \\
\hline
\end{tabular}

\begin{tabular}{|c|c|c|c|c|c|c|}
\hline \\
\hline \multirow{2}{*}{ TRAP DFPTH (in $1000 \mathrm{ft}$} & Shape & \multirow{2}{*}{\begin{tabular}{|l|} 
Level 1-35 \\
3
\end{tabular}} & \multirow{2}{*}{\begin{tabular}{|l|} 
Min \\
10
\end{tabular}} & \multirow{2}{*}{0.5} & \multirow{2}{*}{$\frac{0.05}{16}$} & $\frac{\text { Max }}{1 \varepsilon}$ \\
\hline & \multirow{2}{*}{5} & & & & & \multirow{2}{*}{$\begin{array}{r}18 \\
0.52\end{array}$} \\
\hline $\begin{array}{l}\text { TRAP DEPTH (in } 1000 \mathrm{ft} \text { ) } \\
\text { (from sea level) }\end{array}$ & & \multicolumn{4}{|c|}{ Enter surface to sea level correction $(1000 \mathrm{ft}):$} & \\
\hline
\end{tabular}

\section{NONASSOCIATED GAS ACCUMULATION CHARACTERISTICS}

NA Gas recovery factor $\%$

Type of reservoir-drive (check any that apply):

Natural gas liquids plus condensate to non-associated gas (bbls/million cf) (in place):

70 NGL-NAG=1.785*TD 25.0 (at median)

Nonassociated gas quality parameters:

Hydrogen sulfide \%

CO2 contamination \%

Other inert gases:

$$
\text { Name: }
$$

Name:

Gas fvf

176.7 min depth 281.4 median depth

297.7 max depth
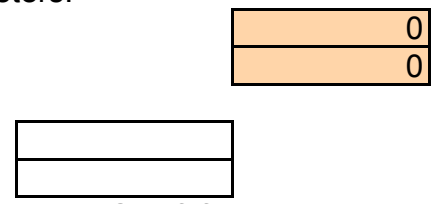

Percent:

Percent:

Gas fvf $=752.2^{*}\left(1-\mathrm{EXP}\left(-0.05728^{*} \mathrm{TD}\right)\right) \quad \mathrm{TD}<=5.67$ thous $\mathrm{ft}$ $113.3+21.1^{*} T D-0.812^{*} \mathrm{TD}^{\wedge} 2+0.0116^{*} \mathrm{TD}^{\wedge} 3 \quad 5.67<\mathrm{TD}<=30$

$\mathrm{TD}=$ trap depth (thous $\mathrm{ft}$ )

For Nonassociated Gas Accumulation:

TIME OF TRAP DEVELOPMENT

STRATIGRAPHIC COMPONENT (Ma)

STRUCTURAL COMPONENT (Ma)

\begin{tabular}{|r|r|r|}
\multicolumn{1}{l}{ BEGIN } & PEAK & \multicolumn{1}{c}{ END } \\
\hline 159 & 135 & 130 \\
\hline 100 & 65 & 25 \\
\hline
\end{tabular}

Assessor's Name: DWH

Date of Data Entry MM/DD/YYYY:

Enter this info on Oil sheet

Date of Simulation Run MM/DD/YYYY: 
Table 1f. Nonassociated gas correlation attributes and engineering computations
Alaskan Assessment Form
14-Oct-04
PLAY: Beaufortian Clinoform

GAS ACCUMULATION ATTRIBUTE DEPENDENCIES

\begin{tabular}{|c|c|c|c|c|}
\hline Correlations & $\begin{array}{l}\text { NET } \\
\text { RESERVOIR } \\
\text { THICKNESS }\end{array}$ & $\begin{array}{l}\text { AREA OF } \\
\text { CLOSURE }\end{array}$ & TRAP FILL & $\begin{array}{l}\text { HYDROCARBO } \\
\text { N PORE VOL }\end{array}$ \\
\hline NET RESERVOIR THICKNESS & $\begin{array}{r}1 \\
\end{array}$ & \begin{tabular}{|r|}
0.0 \\
\end{tabular} & $\begin{array}{r}0.0 \\
\end{array}$ & $\begin{array}{r}0.0 \\
\end{array}$ \\
\hline AREA OF CLOSURE & 0.0 & 1 & 0.0 & 0.0 \\
\hline TRAP FILL & 0.0 & 0.0 & 1 & 0.0 \\
\hline HYDROCARBON PORE VOL & 0.0 & 0 & 0 & 1 \\
\hline
\end{tabular}

Assessor's Name:

Date of Data Entry MM/DD/YYYY:

Date of Simulation Run MM/DD/YYYY:

DWH

$9 / 20 / 04$

$10 / 15 / 04$ 
Table 1g. Shapes to be used to specify accumulation attributes, trap depth, and number of prospects.

(Domains in these examples are 0 to 1 .)

Probability Density

Cumulative Density

Shape 1

$\mathcal{J}$-shapest (mode $=0$ )
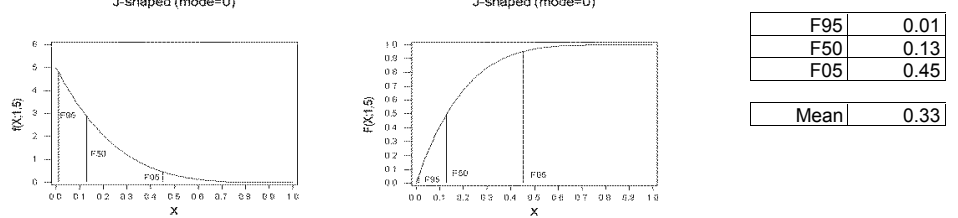

Mean $\quad 0.33$

Shape 2
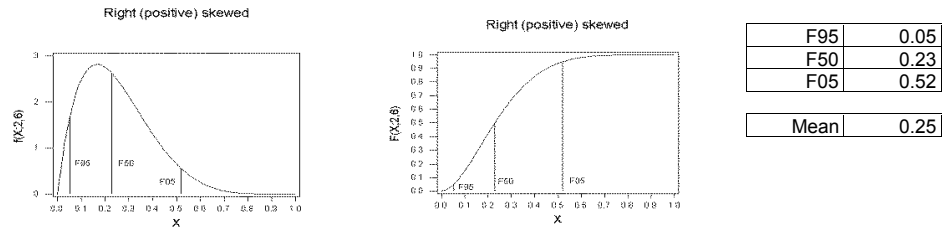

Shape 3
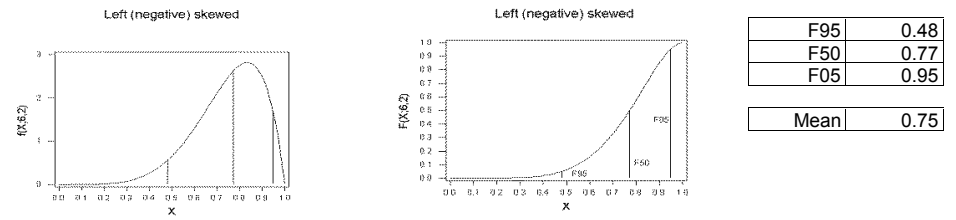

Shape 4
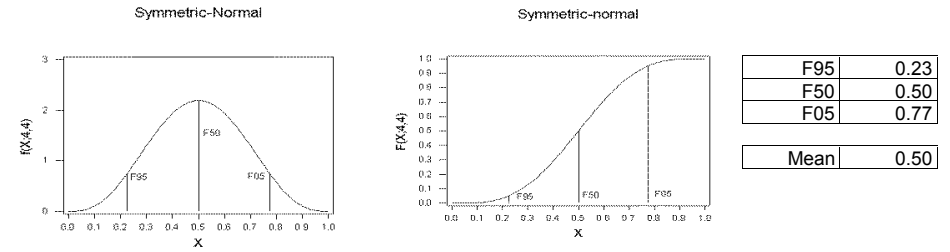

Shape 5
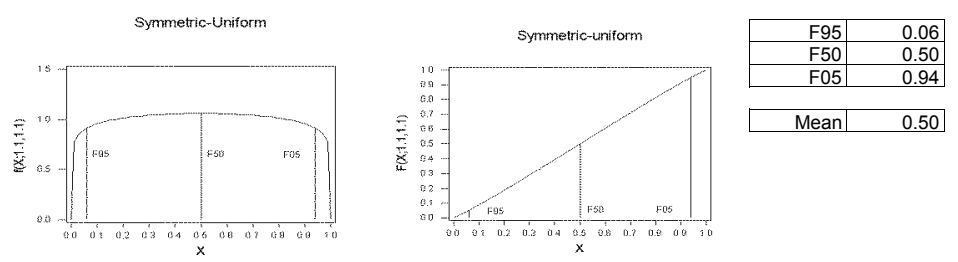

Shape 6 Complement of J (mirror image of shape 1).
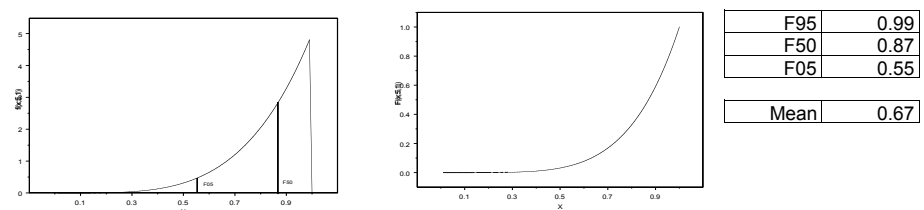

Shape 7 Same as shape 6 except with 0.50 probability at maximum (comp j-1)

Reserved for specification of trap fill
\begin{tabular}{r|r|r|r|r|}
\hline i.e. & LTP & 0.5 & 0.05 & Max \\
\hline 60 & 100 & 100 & 100 \\
\hline
\end{tabular}
Let $X$ be trap fill
If $X<100$ shape is inverted J with Prob (LTP $<=X<100)=0.5$
Prob $(X=100)=0.5$

Shape 8 Same as shape 6 except with 0.05 probability at maximum (comp j-2)

Reserved for specification of trap fill
$\mathrm{X}^{\mathrm{i}}$
\begin{tabular}{r|r|r|r|c|}
\hline i.e. & LTP & 0.5 & 0.05 & Max \\
\hline 60 & 80 & 100 & 100 \\
\hline
\end{tabular}
Let $X$ be trap fill
If $X<100$ shape is inverted J with Prob $($ LTP $<=X<100)=0.95$
Prob $(X=100)=0.05$ 


\section{Oil accumulation attributes}

Assessors specified distributions for the following oil accumulation attribute distributions (table 1a): net reservoir thickness, $N R T$, in feet, area of closure, $A C$, in thousands of acres, porosity, $P O R$, in percent, trap fill, $T F$ in percent, and trap depth, $T D$ in thousands of feet.

Estimates of the lower truncation point (LTP), the $50^{\text {th }}$ (F50), and $5^{\text {th }}$ (F05) fractiles, and the maximum value were entered for $N R T, A C, P O R$, and $T F$.

As specified in the protocol (Appendix B), the LTP was denoted as the minimum size of geologic interest. A principal purpose of the LTP was to eliminate the generation of a container size that would contain a volume of at least the minimum accumulation size (MAS) and have one or more attributes that were too small in a geologic sense. For example, in Table 1a, we note that the LTP for net reservoir thickness (NRT) was 20 feet. It would clearly be possible to generate a container of at least $5 \mathrm{~mm}$ bbl with an $N R T$ of 10 feet, however in the judgment of the assessor, such a field would not be technological recoverable in the foreseeable future. (Note that LTP does not represent a specific fractile common to all attributes in a given play.) It was assumed that for $N R T, A C, P O R$, and $T F$ the population minimum was zero. Assessors were given plots showing the shape of each histogram of the accumulation attributes.

The hydrocarbon pore volume $(H P V)^{2}$, expressed in percent, is one of the components of the equation to estimate in place oil. It is computed as

$$
H P V=P O R-B V W
$$

where $B V W$, the bulk volume water, is defined as the product of $P O R$ and water saturation $\mathrm{S}_{\mathrm{w}}\left(B V W=P O R^{*} S_{w}\right)$. That is, $B V W$ is the fraction or percentage of rock volume, which is filled with water. The question we faced was, what fraction of the rock volume is filled with water (the $B V W$ ) and how much with hydrocarbon (called hydrocarbon pore volume or $H P V)$ ? These two values, given either as a percentage or as a fraction, must sum to the porosity, such that $B V W+H P V=P O R$. The porosity distribution is determined by the assessor from available core data, well logs, and analog fields. Because $B V W$ is the product of porosity and water saturation, the problem of determining $B V W$ reduces to the estimation of water saturation. In this assessment we adopted the same assumption used in the assessment of NPRA and the Arctic National Wildlife Refuge (Nelson, 1999): that $B V W$ is constant in a given field. That assumption rests on the concept that $B V W$ is constant within a specified lithology above the waterhydrocarbon transition zone and implies that water saturation varies inversely with

\footnotetext{
${ }^{2}$ The analysis and text on hydrocarbon pore volume was contributed by Philip Nelson, U.S. Geological Survey.
} 
porosity. The $B V W$ (called $P O R * S w$ on the form) is located in the ATTRIBUTE column of the Oil and Gas attribute assessment sheets (table 1a and table 1e, respectively). The value of $B V W$ is treated as a constant, which is then subtracted from the porosity distribution to yield the distribution of hydrocarbon pore volume. For example, in the Beaufortian Clinoform Play [Oil worksheet], $B V W$ is 6 percent, the median porosity is 13 percent, and consequently the median hydrocarbon pore volume is 7 percent. $B V W$ was between 1 and 6 for the assessed Central North Slope plays.

Low-porosity, fractured reservoirs required a refinement to the estimate of $H P V$. Porosity in fractures can contribute 1 percent, and in some cases 2 or more percent to total porosity. Fractures are considered to be filled with hydrocarbon, because fracture apertures are greater than pore sizes in low-porosity, unfractured rock. Consequently, $B V W_{f}$, the bulk volume water in fractures is zero, and the expression for hydrocarbon pore volume is,

$$
H P V=H P V_{f}+H P V_{u}=P O R_{f}+P O R_{u}-B V W_{u}
$$

where the subscript $f$ represents pore space in fractures and subscript $u$ represents pore space in unfractured rock. Fracture porosity was included in the porosity distribution and bulk volume water was estimated for the unfractured rock; no modification of the worksheet was required for fractured reservoirs.

For $T D$ and number of prospects (table 1c), the LTP is the minimum value of the population distribution. The $T D$ was adjusted before use in the model by adding an average surface-to-sea level elevation correction factor (table 1a for oil and table 1e for gas). Thus, $T D \longleftarrow T D+S_{s l}$, where $S_{\mathrm{sl}}$ is the surface to sea level correction factor $\left(0.24 \times 10^{3}\right.$ feet in the Brookian Clinoform oil play, table 1a)

The oil accumulation attribute distributions were intended to reflect the variation in characteristics of prospects across a play and not variation within a given prospect. The values chosen for the fractiles and other estimates were based upon field studies, geophysical and geochemical data, well logs, and analogy. Specific justifications are given in individual play description reports that are currently in preparation.

\section{Oil accumulation characteristics and estimate of in-place oil}

Samples from these accumulation distributions and an estimate of the formation volume factor $\left(F V F_{o}\right)$ in reservoir barrels/stock tank barrels, rb/stb were combined to estimate $O I P$ (in millions of barrels, $\mathrm{MMBO}$ ), as:

$$
O I P=7.758 \times N R T \times A C \times H P V \times T F \times 10^{-4} / F V F_{o}
$$

By repeated Monte Carlo sampling from the distributions for $N R T, A C, H P V, T F$ and $T D$ we generate a distribution of oil in place. An estimate of $F V F_{o}$, developed by Mahendra Verma, U.S. Geological Survey, (Verma and Bird, 2005) is computed as follows. Let 
$p=1000 T D / 2$ and $t=19 T D+30$ where $p$ is pressure in pounds per square inch (psi) and $t$ is temperature in degrees $\mathrm{F}$. Then the solution specific gravity, sgg is

$s g g=((0.1402 \ln (p+14.7)-0.4227)+(0.1369 \ln (t)+0.0156)+(0.1704 \ln (a g)+0.1469)) / 3$

where $a g$ is API gravity as specified on the oil worksheet play form (table 1a) and "ln" in the above equation is log base e function. The associated uncorrected gas to oil ratio $G O R_{\mathrm{u}}$ (cu. ft. per barrel at stp) is

The corrected GOR is

$$
G O R_{u}=\operatorname{sgg}\left[\frac{(p+14.7) 10^{0.0125 a g}}{18 \times 10^{0.00091 t}}\right]^{1 / 0.83} .
$$

$$
G O R=\left\{\begin{array}{l}
0.86 G O R_{u} \quad G O R_{u} \leq 1200 \\
{\left[0.86+0.24 \sin ^{2}\left(\frac{G O R_{u}-1200}{2250-1200} \frac{\pi}{2}\right)\right] G O R_{u} \quad 1200<G O R_{u}<2250} \\
1.1 G O R_{u} \quad G O R_{u} \geq 2250
\end{array}\right.
$$

Then

$$
\begin{aligned}
o g & =141.5 /(a g+131.5) \\
F & =G O R(\mathrm{sgg} / \mathrm{og})^{0.5}+1.25 t
\end{aligned}
$$

where $o g$ is the specific gravity of oil.

Finally

$$
F V F_{o}=0.972+0.000147 F^{1.175} \text {. }
$$

Oil $F V F$ 's are shown for the API gravities of 23, 34, and 39 (fig. 1).

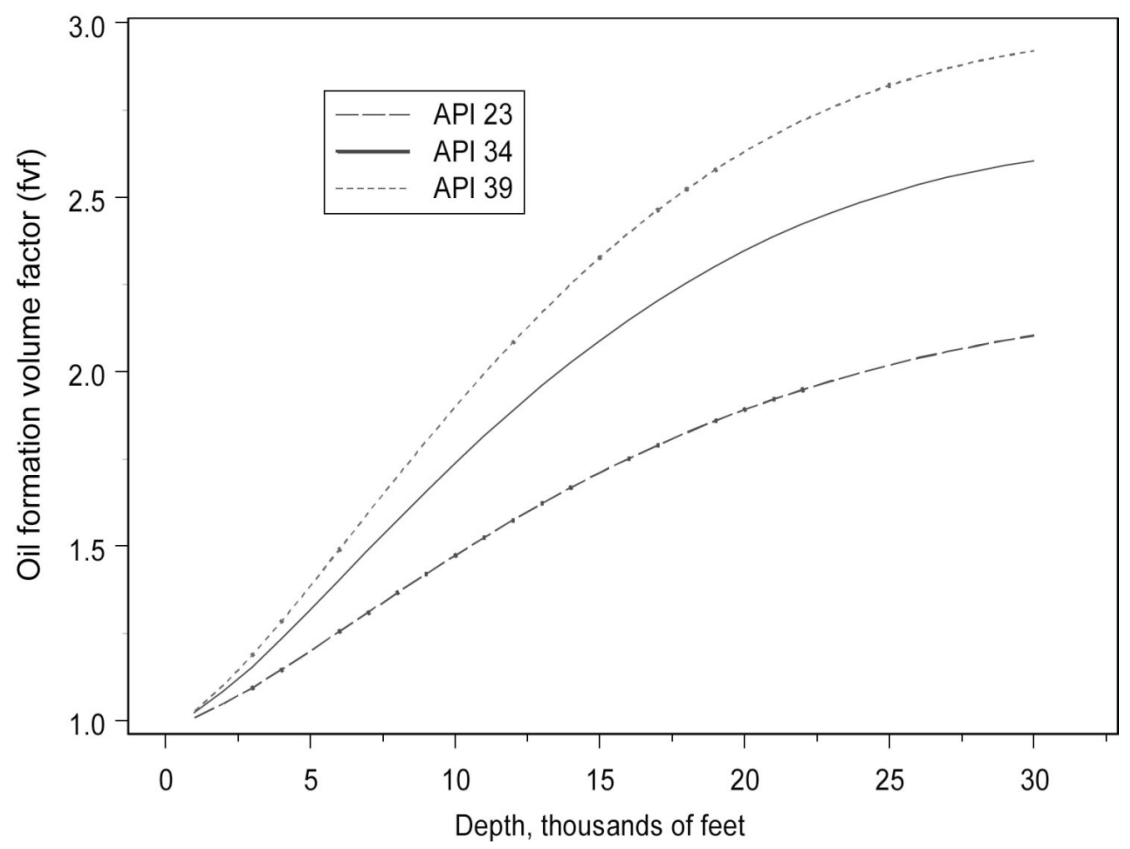

Figure 1. Oil formation volume factor $\left(F V F_{\mathrm{o}}\right)$ versus trap depth $(T D)$ for selected API oil gravities. 


\section{Gas accumulation attributes, characteristics and estimate of in place gas}

The categories of gas oil accumulation attributes and characteristics (table 1e), which were used to compute the accumulation sizes of gas, are the same as for oil, namely $N R T, A C, P O R, T F$, and $T D$. The equation for the accumulation size of nonassociated gas in place, GIP, (in billions of cubic feet, bcf) is:

$$
G I P=4.356 \times N R T \times A C \times H P V \times T F \times 10^{-6} \times F V F_{g}
$$

where $F V F_{g}$ is the formation volume factor for gas. By repeated Monte Carlo sampling from the distributions for $N R T, A C, H P V, T F$ and $T D$ we generate a distribution of nonassociated gas in place. The computational algorithm is the same as that used in NPRA (Schuenemeyer, 2003; Standing, 1977; and Verma and Bird, 2005). We begin by obtaining $z$ (the gas compressibility factor) from graphs (Amyx, Bass, and Whiting, 1960) versus $T D$ (fig. 2).

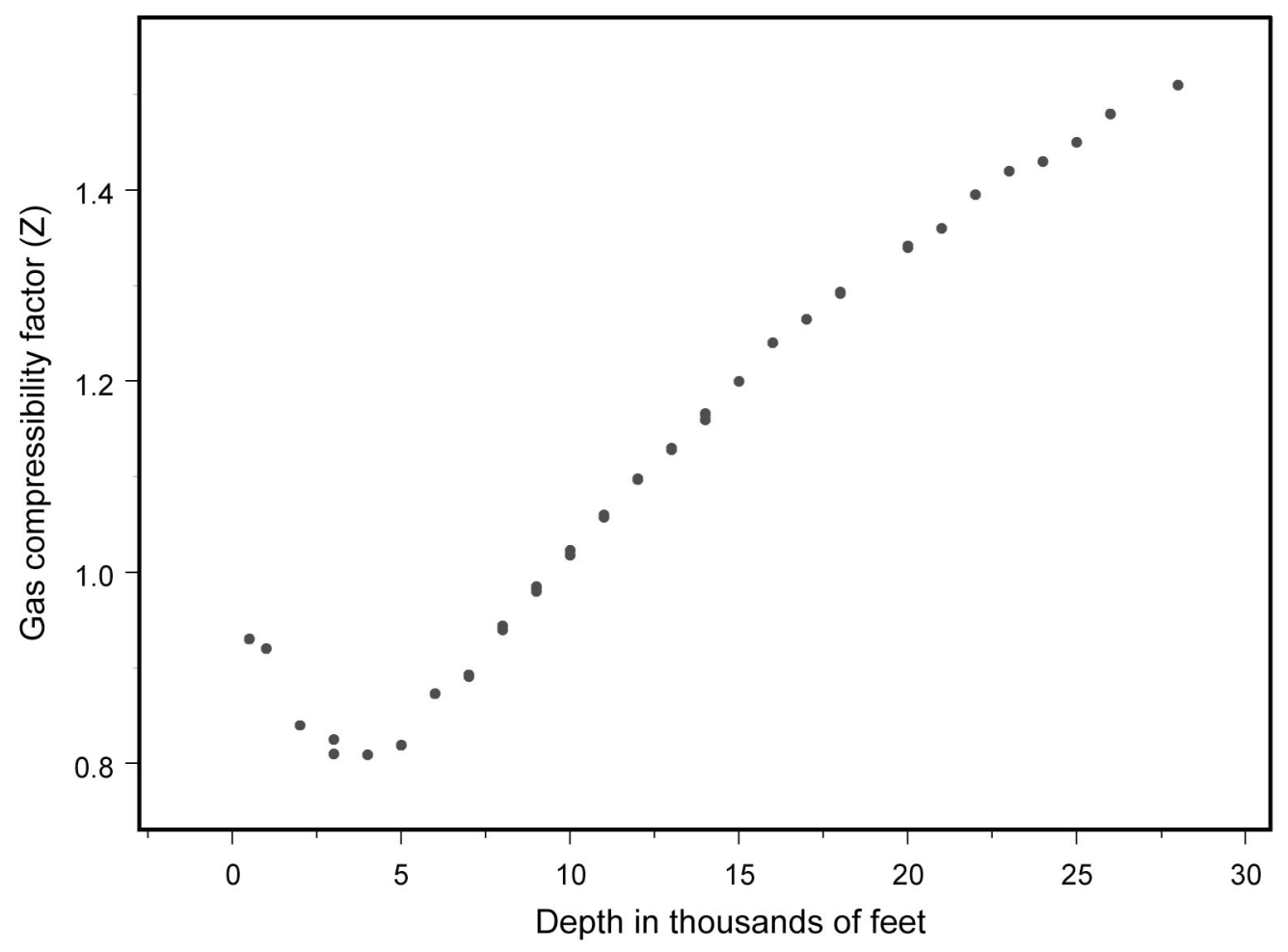

Figure 2. Gas compressibility factor (Z) versus trap depth (TD).

We then compute $F V F_{g}$ as follows.

$$
F V F_{g}=\frac{35.37415(p+14.7)}{(t+460) z}
$$


Finally we smooth using the piecewise curve-fitting model:

$$
F V F_{g} \quad\left\{\begin{array}{l}
752.2\left(1-\mathrm{e}^{-0.05728 T D}\right) \quad 0<T D \leq 5.67 \\
113.3+21.1 T D-0.812 T D^{2}+0.0116 T D^{3} \quad 5.67<T D \leq 30
\end{array} .\right.
$$

The model is illustrated by the solid line in Figure 3.

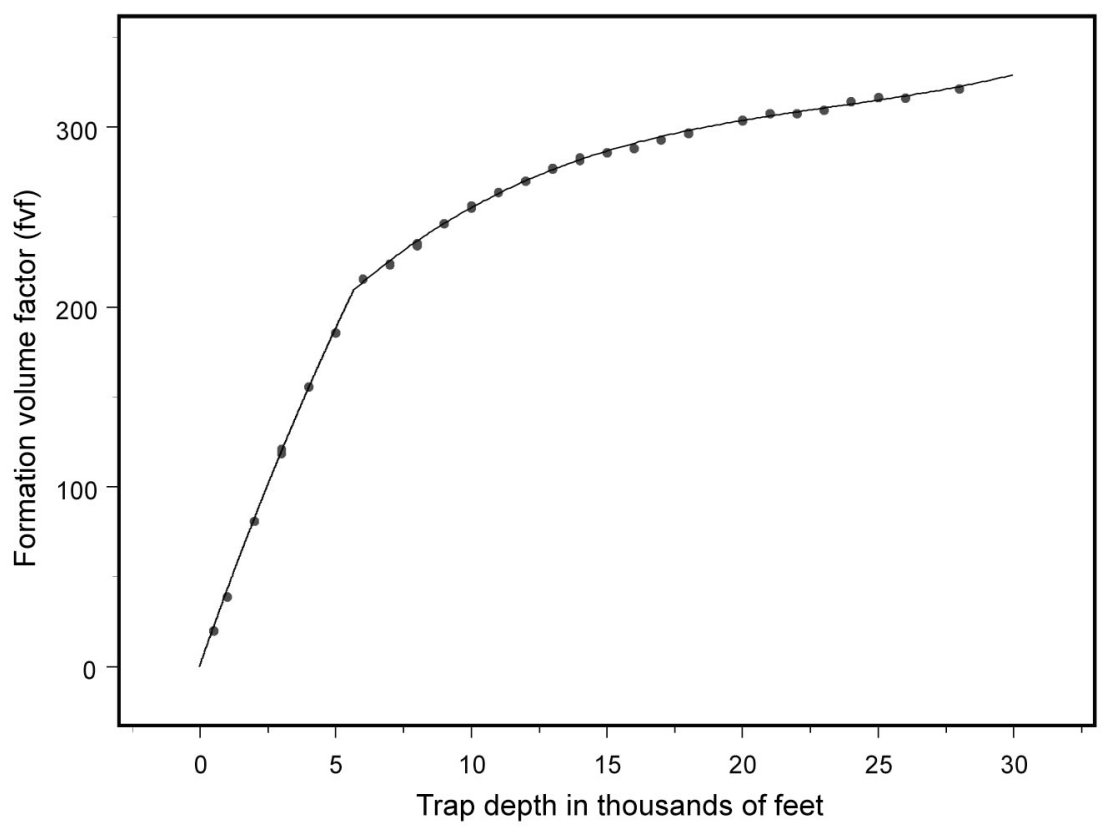

Figure 3. Gas formation volume factor $F V F_{\mathrm{g}}$ versus trap depth $T D$.

\section{Derivatives}

Associated Dissolved Gas (ADG). Given an oil field, it is assumed that associated dissolved gas behaves according to the following model:

ADG $(\mathrm{bcfg})=$ Corrected GOR $(\mathrm{cu} . \mathrm{ft} / \mathrm{bbl}) * 0.001 *$ Recov Oil $(\mathrm{mm} \mathrm{bbl})$

Natural Gas Liquid.

\section{For associated gas:}

$\mathrm{NGL}-\mathrm{ADG}(\mathrm{mm} \mathrm{bbl})=\mathrm{ADG} * \mathrm{NGLR} * 0.001$

where

NGLR $=3.3523 \exp (0.1850 T D)\left(\mathrm{bbl} /\right.$ million $\mathrm{ft}^{3}$ at STP $) T D \leq 16$ and $T D$ is trap depth in thousands of feet. 


\section{For nonassociated gas:}

NGL-GAS $(\mathrm{mm}$ bbl $)=$ RNAG * NGL-NAG * 0.001

where RNAG is recoverable nonassociated natural gas in bcf and

NGL-NAG $=\left\{\begin{array}{ll}1.3 T D & T D \leq 10 \\ 13 & T D>10\end{array}\left(\mathrm{bbl} /\right.\right.$ million $^{3}{ }^{3}$ at STP $)$

Note that the temperature gradients are assumed to be $1.3^{\circ} \mathrm{F} / 100 \mathrm{ft}$ for gas plays and $1.9^{\circ} \mathrm{F} / 100 \mathrm{ft}$ for oil plays. See Verma and Bird (2005) for details on petroleum engineering models.

\section{Correlated attributes}

For some plays assessor chose to specify non-zero pairwise correlations between sets of the attributes NRT, $A C, P O R, T F$ and $T D$ in Table $1 \mathrm{~b}$ (oil) and/or Table $1 \mathrm{f}$ (gas). These correlations, which were specified individually for oil and gas, ranged from -0.75 to 0.30. For example, in the Brookian Clinoform Structural North play, the correlations specified by the assessor were:

\begin{tabular}{|l|r|r|r|r|}
\cline { 2 - 5 } \multicolumn{1}{c|}{ Correlations } & $\begin{array}{c}\text { NET RESERVOIR } \\
\text { THICKNESS }\end{array}$ & $\begin{array}{c}\text { AREA OF } \\
\text { CLOSURE }\end{array}$ & TRAP FILL & $\begin{array}{c}\text { HYDROCARBON } \\
\text { PORE VOL }\end{array}$ \\
\hline NET RESERVOIR THICKNESS (NRT) & 1 & 0.3 & 0.0 & -0.5 \\
\hline AREA OF CLOSURE (AC) & 0.3 & 1 & 0.0 & 0.0 \\
\hline TRAP FILL (TF) & 0.0 & 0.0 & 1 & 0.0 \\
\hline HYDROCARBON PORE VOL (HPV) & -0.5 & 0 & 0 & 1 \\
\hline
\end{tabular}

The positive correlation of 0.3 between NRT and AC would mean that given a high (low) value of NRT we would be slightly more likely to select a high (low) value of AC. The negative correlation between NRT and HPV would mean that given a high (low) value of NRT we would be more likely to select a low (high) value of HPV. Thus, rather than the sampling being independent, when a pairwise correlations is specified, a sample from the second attribute is conditioned on a value from the first. Because some of the user specified distributions were highly skewed, the multivariate sampling was nonparametric and used ranks. So for example, if we were to do 10 simulations, we would draw 10 random variates from the NRT distribution, sort them in ascending order, and assign them ranks from 1 to 10 . We repeat the same process for $\mathrm{AC}$, however, the ranks assigned to AC may be $\{4,3,7,2,10,6,1,10,5,8\}$. The correlations between the ranks assigned to NRT and those assigned to $\mathrm{AC}$ is 0.3 , and thus values of $\mathrm{AC}$ would be correlated with those of NRT at approximately the same level.

The algorithm used to incorporate these correlations structures into the oil and gas accumulation formulas is as follows: 
1. Let $\mathrm{R}_{\mathrm{c}}$ be a matrix whose elements are the assessor specified pairwise correlations. We assume $\mathrm{R}_{\mathrm{c}}$ to be a 5 x 5 matrix. Also, let $n s$ be the number of simulation runs.

2. We then see if $R_{c}$ is permissible. A permissible matrix is one whose determinant is greater than or equal to zero. All assessor defined correlations resulted in permissible matrices. (See additional discussion in the section on Aggregation Methodology.)

3. If $R_{c}$ is permissible, let $C_{h}=$ Cholesky $\left(R_{c}\right)$, be the Cholesky factorization of $R_{c}$.

4. Let $\mathrm{U}_{\mathrm{a}}$ be a $n s \times 5$ matrix, where each row of $\mathrm{U}_{\mathrm{a}}$ is an independent set of uniform random numbers between -1 and 1 .

5. Let $\mathrm{U}_{\mathrm{ac}}=\mathrm{U}_{\mathrm{a}} \times \mathrm{C}_{\mathrm{h}}$.

6. Let $\mathrm{Ur}_{[, \mathrm{i}]}=\operatorname{Rank}\left(\mathrm{U}_{\mathrm{ac}[\mathrm{i}]}\right), i=1, \ldots, 5$

7. The ranked values in the columns of Ur represent the sample numbers of the accumulations (with accumulations sorted in ascending order) necessary to achieve the desired correlation structure as specified in $R_{c}$.

This methodology was implemented in Splus function asoga.fnt, called from Splus function AMsim.fn. For additional mathematical and computational details see Kotz, Balakrishnan, and Johnson (2000) and Stewart (1973).

\section{Risking}

Risk in the context of this study is the probability that a play or prospect would be unsuccessful because of the failure of one or more geologic attributes necessary to achieve success. Because it is natural to think of the likelihood of an attribute being present, we used the complement of risk, namely favorability. A favorability of one implies zero risk.

There are two favorability structures. One is play; the other is prospect. Prospect favorability was further subdivided into oil and gas. Each of these is the product of three attributes, however, play favorability refers to the product of attributes needed for a successful play, whereas, prospect probability refers to the product of those attributes associated with a randomly chosen prospect. The attributes that constitute these structures are charge, trap, and timing formation. Although the names of the attributes are the same at the play and prospect levels, there are nine distinct attributes. They are assumed to be pairwise independent of each other. Prospect attributes were assessed conditional upon the play being successful. Thus, in the Beautfortian Clinoform (table $1 d)$, the play favorability is

$$
\begin{aligned}
F_{P} & =C \times T \times F \\
& =1 \times 0.6 \times 1 \\
& =0.6
\end{aligned}
$$

where charge $(\mathrm{C})$, trap/rock $(T)$, and timing $(F)$ are at the play level. In the simulation, if we draw $\mathrm{u}$, a $[0,1]$ uniform random number, and $u \leq F_{P}=0.6$, we deem the play successful. Prospect favorability is specified for oil and gas separately. Again in the Beautfortian Clinoform (table 1d), the prospect favorability for oil is 


$$
\begin{aligned}
F_{o} & =c_{o} \times t_{o} \times f_{o} \\
& =0.9 \times 0.2 \times 1 \\
& =0.18
\end{aligned}
$$

where charge $\left(c_{\mathrm{o}}\right)$, trap/rock $\left(t_{o}\right)$, and timing $\left(f_{o}\right)$ are at the prospect level for oil. In the Beaufortian Clinoform (table 1d), the prospect favorability for gas is

$$
\begin{aligned}
F_{g} & =c_{g} \times t_{g} \times f_{g} \\
& =0.9 \times 0.2 \times 1 \\
& =0.18
\end{aligned}
$$

where charge $\left(c_{\mathrm{g}}\right)$, trap/rock $\left(t_{g}\right)$, and timing $\left(f_{g}\right)$ are at the prospect level for gas. In this instance both prospect favorability values are the same. In general, they will not be. In the simulation, we draw separately from the number of prospects for oil and gas (table 1c). For each oil prospect, we draw $u$, a $[0,1]$ uniform random number, and if $u \leq F_{o}=0.18$, we deem the prospect successful. Likewise, for each gas prospect we draw $u$, a $[0,1]$ uniform random number, and if $u \leq F_{o}=0.18$, we deem the prospect successful.

A successful play is one in which all three of the play level attributes necessary for a prospect of at least $5 \mathrm{~mm}$ bbl technically recoverable oil or 100 bcf technically recoverable nonassociated gas are present. However, there is no guarantee that such a prospect will be found in a "successful play". A failure to draw at least one deposit in a "successful play" can occur when few prospects are specified and/or the prospect favorability is low.

Assessment definitions (Appendix C) were established and made available to the assessors to provide specific guidelines to allow them to differentiate between these two risks.

\section{Graphical Feedback}

Plots of the accumulation attribute distributions, trap depth, and number of prospects distributions, and corresponding fitted values to beta distributions were provided to the assessors. Table 2 shows graphs of area of closure and number of prospects for the oil component of the Beaufortian Clinoform play (table 2a). These graphs could be viewed immediately after data entry. Fitted distributions (Table $2 b$ ) from the Splus nonlinear fitting distributions used in the play simulation were provided to the assessors in batch mode. Box plots of accumulation attributes that constituted prospect volumes near the MAS (not shown) were also provided. Finally the oil accumulation distribution was given in original units (table $2 \mathrm{c}$ ) and in logs (table $2 \mathrm{~d}$ ). In the original units, the accumulation graph only extends to the F05 value in order to allow the assessor 
Table 2a. Instantaneous feedback graphs, Beaufortian Clinoform play.

Oil Graphs

Density, LTP, and complement cumulative distribution function (ccdf)
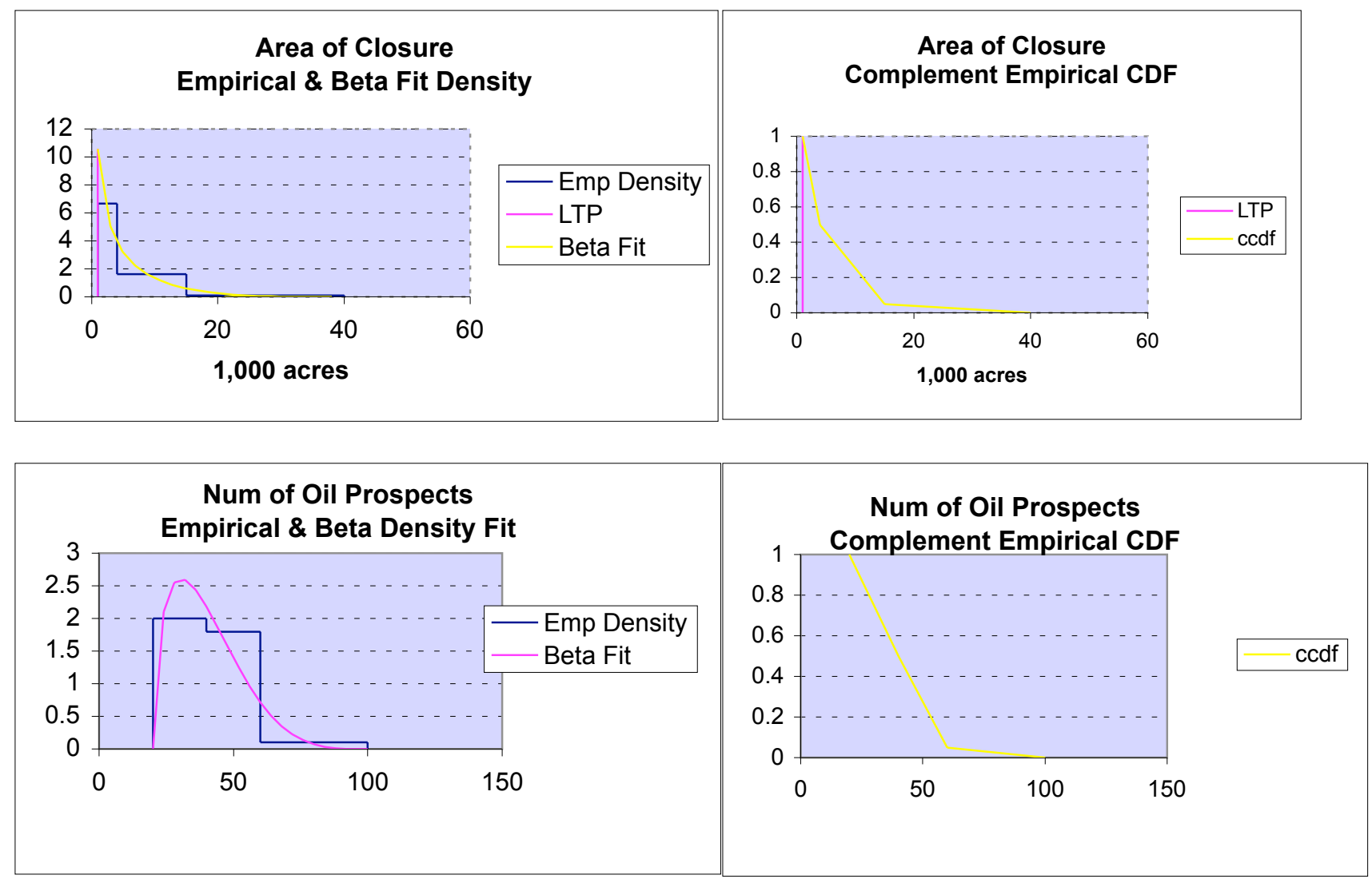
Table 2b. Model fitted beta distributions to oil and nonassociated gas attributes, Beaufortian Clinoform play.

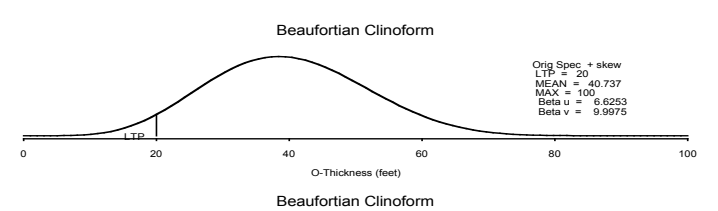

Beaufortian Clinoform

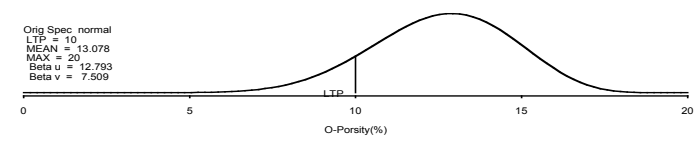

Beaufortian Clinoform
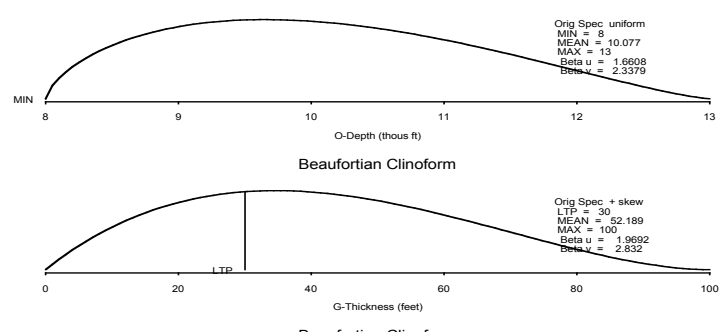

Beaufortian Clinoform

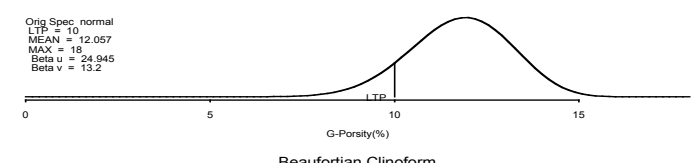

Beaufortian Clinoform

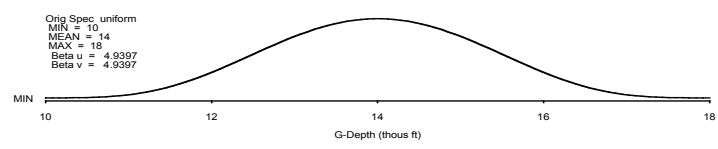

Beaufortian Clinoform
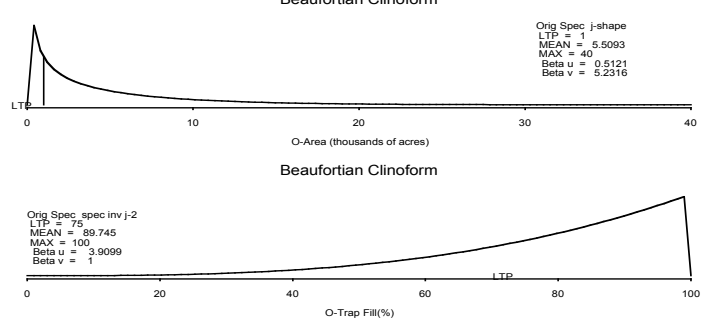

Beaufortian Clinoform
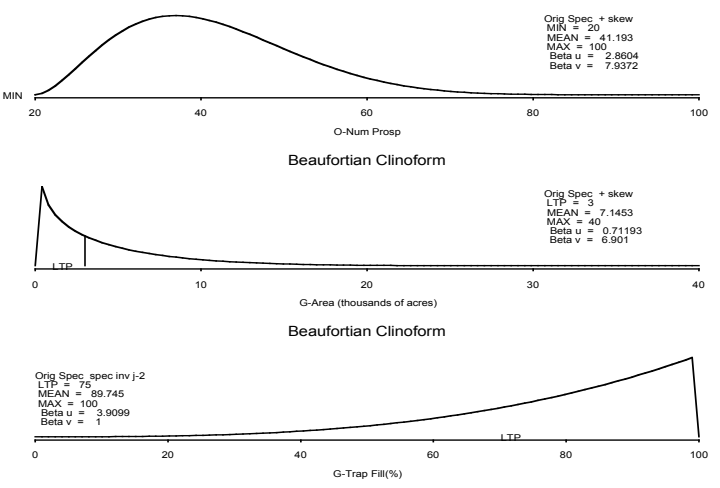

Beaufortian Clinoform

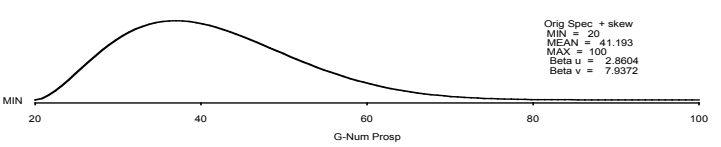


Table 2c. Oil accumulation distribution, Beaufortian Clinoform play.

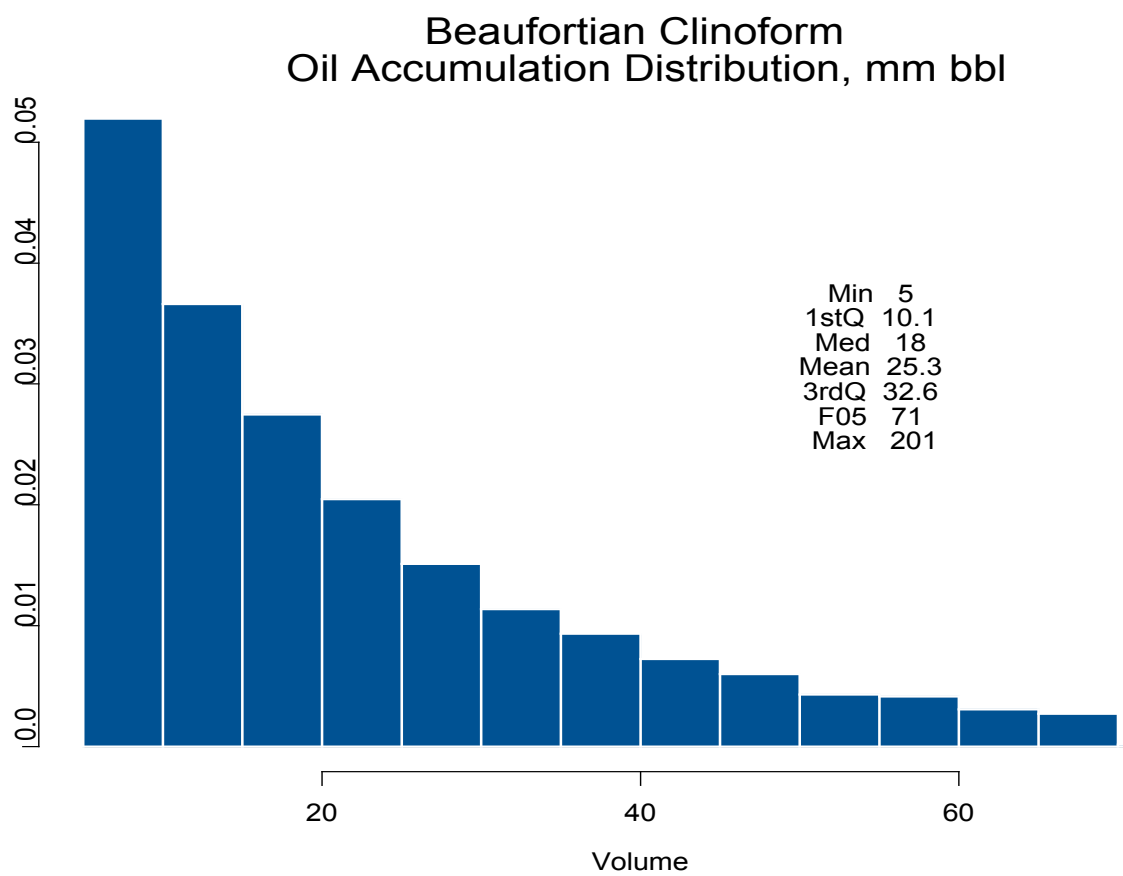

Table 2d. Oil accumulation distribution, Beaufortian Clinoform play, log units.

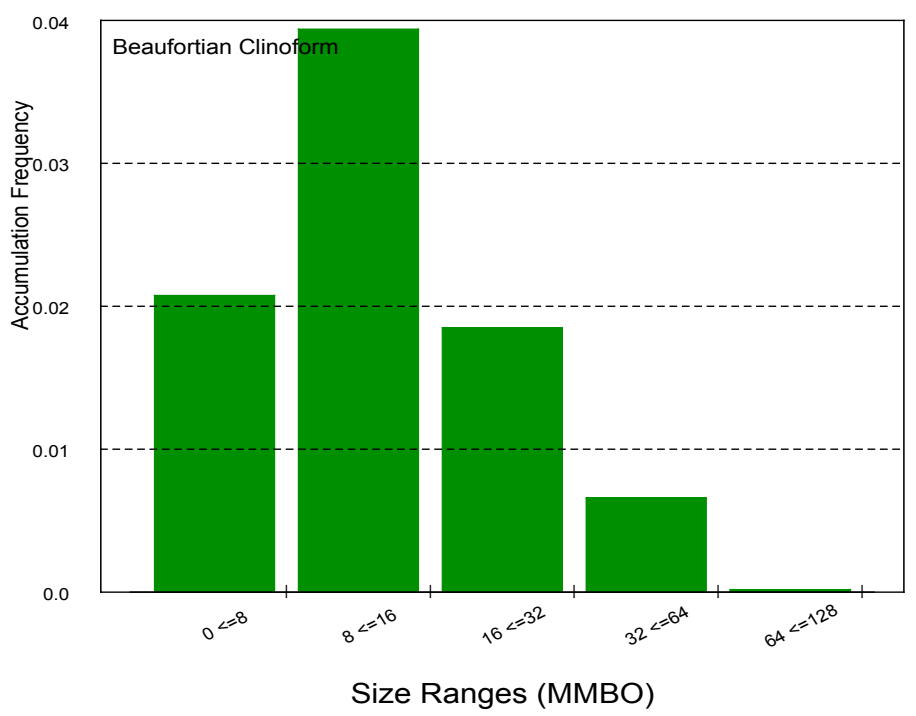


to focus on the left component of the distribution. The accumulation distribution in log units was presented to help the assessor estimate the number of smaller undiscovered fields.

\section{The Deposit Simulation}

The methodology was based upon a Monte Carlo simulation. A series of functions, written in Splus6.1, described in Appendix A, and listed in Appendix D were used to implement the simulation. For each play, 10,000 simulations were run, conditioned on the play being favorable. For example, the Beaufortian Clinoform play favorability probability was 0.60 . The expected total number of runs would be 16,667 $(10,000$ divided by 0.60$)$. However, since the expected number of unsuccessful runs was 6,667 , we chose to run only the 10,000 potentially successful runs. The reason for choosing to run 10,000 simulations conditioned on a successful play was to obtain similar levels of precision on the summary statistics for all plays, even those that were highly risked. The uniform random number generator used for the simulations is the Splus6.1 function runif. A list of plays and play favorability probability is given in Table 3 (this is the same as Splus file AMplayr).

Table 3. Play number, name, favorability probability (risk), and file name.

\begin{tabular}{|c|l|c|l|}
\hline $\begin{array}{c}\text { Play } \\
\text { number }\end{array}$ & \multicolumn{1}{|c|}{ Play name } & $\begin{array}{c}\text { Play } \\
\text { favorability }\end{array}$ & $\begin{array}{c}\text { Splus file } \\
\text { name }\end{array}$ \\
\hline 1 & Brookian Clinoform & 1.0 & BkC \\
2 & Brookian Topset & 1.0 & BkT \\
3 & Beaufortian Upper Jurassic Topset East & 0.6 & BeUJTE \\
4 & Beaufortian Upper Jurassic Topset West & 1.0 & BeUJTW \\
5 & Beaufortian Clinoform & 0.6 & BeC \\
6 & Beaufortian Kuparuk Topset & 1.0 & BeKT \\
7 & Beaufortian Cretaceous Shelf Margin & 1.0 & BeCSM \\
8 & Triassic Barrow Arch & 1.0 & BaAT \\
9 & Ivishak Barrow Flank & 0.8 & BaFI \\
10 & Endicott & 0.9 & End \\
11 & Endicott Truncation & 1.0 & EndT \\
12 & Franklinian & 1.0 & Frk \\
13 & Lisburne Barrow Arch & 1.0 & LBA \\
14 & Lisburne Barrow Flank & 0.9 & LBF \\
15 & Kemik-Thompson & 1.0 & KTh \\
16 & Basement Involved Structural & 1.0 & BIS \\
17 & Beaufortian Structural & 0.9 & BeS \\
18 & Brookian Clinoform Structural South & 1.0 & BkCSS \\
19 & Brookian Clinoform Structural North & 1.0 & BkCSN \\
20 & Brookian Topset Structural South & 1.0 & BkTSS \\
21 & Brookian Topset Structural North & 1.0 & BkTSN \\
22 & Thrust Belt Triangle Zone & 1.0 & TBTZ \\
23 & Thrust Belt Lisburne & 1.0 & TBL \\
24 & Ellesmerian structural & 0.9 & ES \\
\hline
\end{tabular}


Computation was done with a series of Splus v6.1 functions (Insightful Corp., Seattle, WA). (Splus is a commercially available statistical computing package and programming language, which is functional in form and can be translated into other high level languages, especially $\mathrm{R}^{3}$ statistical software. Input from tables $1 \mathrm{a}-1 \mathrm{f}$ was automatically transferred to an Excel worksheet (WorkS1) (not shown), which provided input to Splus function AMdata.fn.

The fractiles for hydrocarbon accumulation attributes, trap depth, and number of prospects specified by assessors for oil and gas were each fit to a two parameter beta distribution

$$
f(x ; u, v)=B(u, v) x^{u}(1-x)^{v} \quad 0 \leq x \leq 1
$$

using Splus function AMpare.fn. The method of fit was the Splus nonlinear estimation function nlmin, which uses a general quasi-Newton optimizer. The function being minimized (fmin) was

$$
(q \operatorname{beta}(0.50, p[1], p[2])-c o[1])^{2}+(q \operatorname{beta}(0.95, p[1], p[2])-c o[2])^{2}
$$

where qbeta is the Splus beta quantile function, $p[1]$ and $p$ [2] are initial estimates of the beta distribution parameters established from the user specified distribution shape (table $1 \mathrm{~g}$ ), and $c[1]$ and $c$ [2] are the standardized (to 0 to 1 ) values of the 0.50 (F50 fractile) and 0.95 (F05 fractile) percentiles specified by the assessors. The result is estimated parameters of the beta distribution. The use of a modified beta distribution only occurred in a few instances for the distribution of trap fill where an assessor specified a) an inverted $\mathrm{j}$-shaped distribution with 0.50 probability occurring at the maximum value (table $1 \mathrm{~g}$, shape 7 ), or b) an inverted $\mathrm{j}$-shaped distribution with 0.05 probability occurring at the maximum value table $1 \mathrm{~g}$, shape 8 ). In case a) the fitted beta density function was

$$
f(x ; 5,1)=\left\{\begin{array}{l}
0.5 \operatorname{beta}(x ; 5,1), \quad 0 \leq x<1 \\
0.5 \quad x=1
\end{array}\right.
$$

In case $b$ the fitted beta distribution was

$$
f(x ; 1, v)=\left\{\begin{array}{l}
0.95 \operatorname{beta}(x ; 1, v), \quad 0 \leq x<1 \\
0.05 \quad x=1
\end{array}\right.
$$

where $v=\ln (0.5) / \ln \left(1-x_{0.95}\right)$. Assessors were given the option of choosing other distributions if they did not feel these provided an adequate fit, however, none did.

\footnotetext{
${ }^{3}$ The R-Project for Statistical Computing, http://www.r-project.org/. R is open source software.
} 
Assessors were also provided feedback in the form a graphical representation of the beta distribution as fit from their fractile specification. Changes to fractile specifications were allowed, as needed, to properly reflect the geology.

The beta distribution was chosen because assessor specified distribution ranged from highly right skewed to symmetric to left skewed, and from peaked to flat. The beta distribution was able to accommodate this wide range of shapes. In the simulation, all sampling was from the fitted beta distributions. Note assessors could specify that certain beta distributions be correlated. Correlated samples, when specified by assessors, were incorporate into the oil and/or gas in place accumulation distributions, as previously described.

Figure 4 is a flow chart for the simulation algorithm (function AMsim.fn). It begins with the main simulation loop, which was executed 10,000 times for each play; 10,000 being the expected number of potentially favorable plays. Next, a sample was taken from the number of prospects distribution. The number of prospect distribution for oil is independent of that for gas for purposes of sampling. The same assessor typically specified both distributions so in that sense they would not be independent, however, this factor was not incorporated into the simulation. The number of prospects is oil of at least $5 \mathrm{~mm}$ bbl technically recoverable, and nonassociated gas of at least 100 bcf technically recoverable. The oil or gas prospect probability was then applied. For a successful prospect, now relabeled a deposit, a value of at least the MAS was obtained via a random sample from the oil or gas in place accumulation distribution. Associated-dissolved gas and natural gas liquids (NGL) from associated-dissolved gas and from nonassociated gas were also computed. Technically recoverable quantities of oil were computed by multiplying the in-place volumes by the oil recovery factor (table 1a and table 1e). Function AMplay.fn totals field level data in each simulation. The output file will contain one line per simulation run. Function AMpsum.fn yields numeric and graphical summary statistics by play. Size-frequency distributions for recoverable oil and nonassociated gas were computed and graphed using functions (AMfreq.fn and AMsf.fn). The purpose of these functions is to allow the assessor to see the result of his/her attribute specification prior to a final simulation run. An example of the detailed output from function AMsim.fn is given in Table 4. 
Figure 4. General flow chart for simulation algorithm.

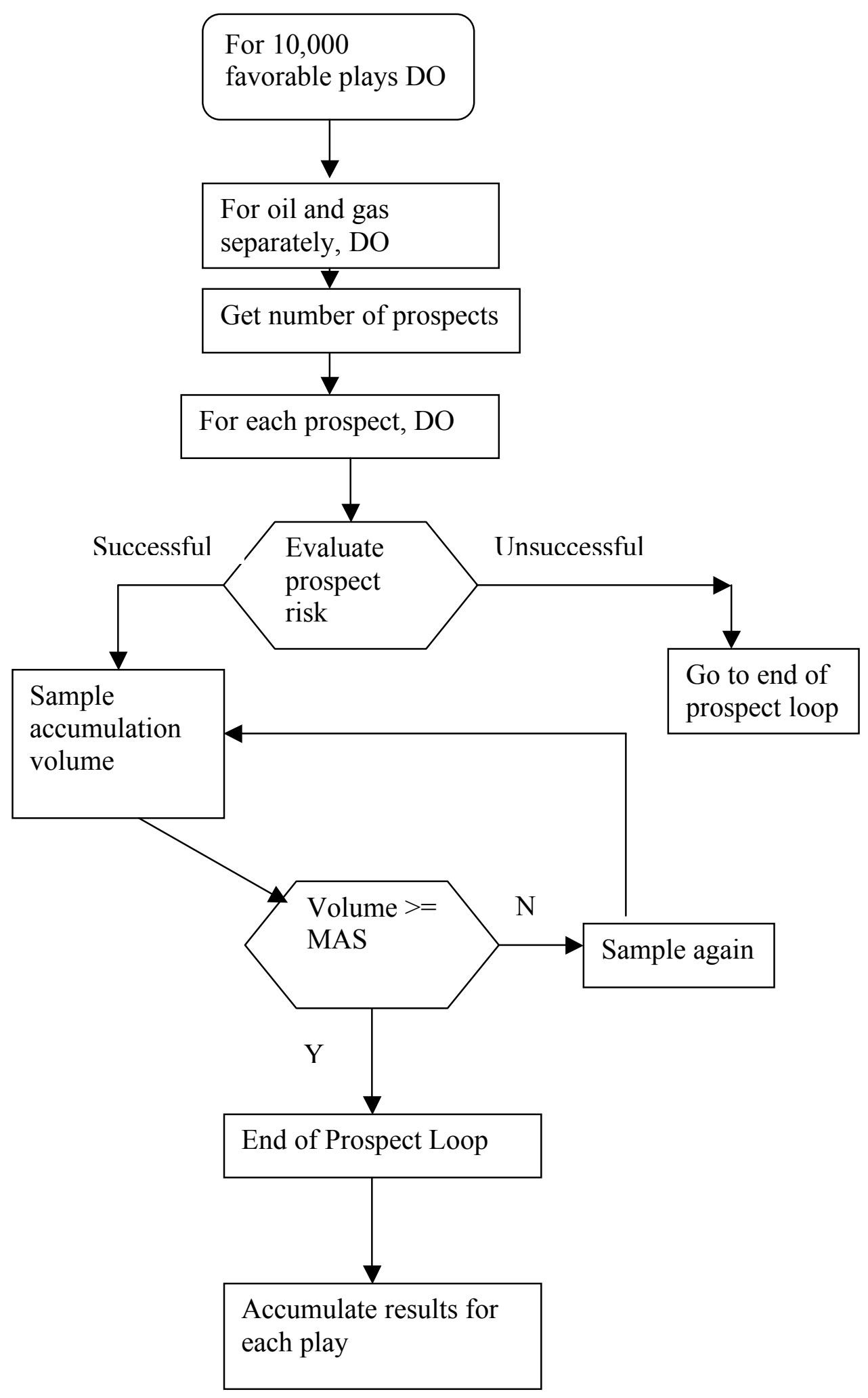


Table 4. Sample simulation output from the Beaufortian Clinoform play.

[run, 1 to number of simulations; numdep, number of deposits this run; depnum, deposit number from 1 to numdep; og.id, 1 for oil, 2 for nonassociated gas; rec.og, technically recoverable oil or gas; nrt, net reservoir thickness in feet; ac, area of closure in thousands of acres; hpv, hydrocarbon pore volume in percent; tf, trap fill in percent; td, trap depth in thousands of feet; fvf, formation volume factor; rec.agas, recoverable associated dissolved gas in billions of cubic feet; rec.NGL, recoverable natural gas liquid in millions of barrels]

\begin{tabular}{|r|r|r|r|r|r|r|r|r|r|r|r|r|}
\hline run & numdep & depnum & og.id & rec.og & nrt & ac & hpv & tf & td & fvf & rec.agas & rec.NGL \\
\hline 1 & 14 & 1 & 1 & 34.21 & 48.31 & 4.80 & 9.69 & 86.82 & 9.26 & 1.77 & 44.53 & 0.83 \\
\hline 1 & 14 & 2 & 1 & 28.10 & 52.88 & 5.53 & 6.02 & 96.54 & 10.10 & 1.88 & 40.80 & 0.89 \\
\hline 1 & 14 & 3 & 1 & 13.01 & 50.54 & 3.63 & 4.56 & 94.43 & 10.15 & 1.88 & 19.00 & 0.42 \\
\hline 1 & 14 & 4 & 1 & 32.11 & 42.55 & 7.83 & 6.24 & 93.28 & 10.07 & 1.87 & 46.44 & 1.00 \\
\hline \hline 1 & 14 & 1 & 2 & 312.21 & 66.00 & 9.91 & 5.54 & 99.54 & 14.50 & 283.88 & $\mathrm{NA}$ & 4.06 \\
\hline 1 & 14 & 2 & 2 & 163.06 & 62.81 & 4.24 & 8.01 & 87.24 & 15.34 & 287.78 & $\mathrm{NA}$ & 2.12 \\
\hline 1 & 14 & 3 & 2 & 376.85 & 32.76 & 22.93 & 5.87 & 98.41 & 14.72 & 284.97 & $\mathrm{NA}$ & 4.90 \\
\hline 1 & 14 & 4 & 2 & 342.07 & 60.30 & 11.90 & 6.19 & 90.84 & 13.41 & 278.19 & $\mathrm{NA}$ & 4.45 \\
\hline 1 & 14 & 5 & 2 & 409.18 & 59.78 & 15.86 & 5.49 & 89.93 & 15.06 & 286.53 & $\mathrm{NA}$ & 5.32 \\
\hline 1 & 14 & 6 & 2 & 102.54 & 54.82 & 3.43 & 6.60 & 97.22 & 13.56 & 279.05 & $\mathrm{NA}$ & 1.33 \\
\hline
\end{tabular}

\section{To obtain}

$\mathrm{p}($ pressure, $\mathrm{psi})=1000 * \mathrm{td} / 2$

$\mathrm{t}($ temp, $\operatorname{deg} \mathrm{F})=19^{*} \mathrm{td}+30$

$z$ (gas compressibility, na gas $)=35.37415^{\star}(p+14.7) /\left(\mathrm{fvfg}^{\star}(\mathrm{t}+460)\right)$

where fvfg is fvf nonassociated gas

inplace oil at sea level $=$ og.id(1)/(oilr * .01)

inplace nonassociated gas at sea level $=$ og.id $(2) /($ gasr * .01$)$

where og.id(1) is technically recoverable oil

og.id(2) is technically recoverable gas

oilr is oil recovery factor in percent (from play sheets)

gasr is nonassociated gas recovery factor in percent (from play sheets) 


\section{Aggregation Methodology}

\section{Overview}

Resource estimates from the 24 individual plays were aggregated to total resources in the Central North Slope assessment area. An aggregate distribution was constructed by sampling from the individual plays to estimate assessor specified dependencies between plays. These dependencies result from shared sources of charge, trap and/or timing. Dependency does not affect the mean of the aggregate distribution, only the spread. The mean of the aggregate is simply the sum of the means of the plays to be aggregated. The basic concern in aggregating results is the effect that dependency has upon the spread of the aggregate distribution and thus on estimates of uncertainty. Failure to account for positive dependency would have resulted in estimates of uncertainty that were too narrow and thus would have created a higher level of confidence in results than would be warranted if the correct measure of dependency were used.

The basic procedure used was to create a correlation matrix from assessorspecified dependencies, generate observations that have the specified correlation structure, rank the correlations, and then choose the samples to form an aggregate distribution. The chosen samples are based upon rank order of the BOE in each play.

\section{Specifying the Dependency}

Assessors considered all possible pairs of the 24 plays being assessed. For each pair they assigned one of three values $(0.1,0.5$, and 0.9$)$, corresponding to low, medium, and high correlation to the attributes of charge, trap, and timing (table 5a-5c). A high (positive) value assigned to charge between, say plays A and B may indicate a common mechanism charged both plays. Thus, if the value of charge in play A was found to be high, the value of charge in play B would most likely be high. A single correlation matrix (table 5d) was then formed by taking the arithmetic average of the three correlation matrices.

There is a potential inconsistency associated with specifying correlations by pairs of plays, namely, some correlations impose restrictions on others. For example, suppose the correlation between plays $\mathrm{A}$ and $\mathrm{B}$ is 0.367 and that between plays $\mathrm{A}$ and $\mathrm{C}$ is 0.500 . Then the range of the correlation between plays $\mathrm{B}$ and $\mathrm{C}$ is restricted in that not all values between -1 and +1 are permissible. In order to see if the $24 \times 24$ computed correlation matrix (table 5d) was permissible, a statistical procedure called eigenvalue analysis was performed (function AMcorr.fn). The minimum eigenvalue of a permissible (positive definite) correlation matrix must be greater than zero. The minimum eigenvalue of this matrix was -0.110 . Thus, a slight biasing factor, 0.111 , was applied to each of the 24 eigenvalues. Then the correlation matrix was reconstructed (table 5e). The maximum adjustment made to correlations after application of the bias factor was 0.09 , however, most pairwise adjustments were 0.01 . These adjustments were judged to be within the 
Table 5a. Assessor specified Charge dependencies between plays.

[Correlations: 0.1 , low; 0.5 , medium; 0.9 , high]

\begin{tabular}{|c|c|c|c|c|c|c|c|c|c|c|c|c|c|c|c|c|c|c|c|c|c|c|c|c|}
\hline & & 1 & 2 & 3 & 4 & 5 & 6 & 7 & 8 & 9 & 10 & 11 & 12 & 13 & 14 & 15 & 16 & 17 & 18 & 19 & 20 & 21 & 22 & 23 \\
\hline 1 & Brookian Clinoform & & & & & & & & & & & & & & & & & & & & & & & \\
\hline 2 & Brookian Topset & 0.9 & & & & & & & & & & & & & & & & & & & & & & \\
\hline 3 & Beaufortian Upper Jurassic Topset East & 0.1 & 0.1 & & & & & & & & & & & & & & & & & & & & & \\
\hline 4 & Beaufortian Upper Jurassic Topset West & 0.1 & 0.1 & 0.9 & & & & & & & & & & & & & & & & & & & & \\
\hline 5 & Beaufortian Clinoform & 0.1 & 0.1 & 0.9 & 0.9 & & & & & & & & & & & & & & & & & & & \\
\hline 6 & Beaufortian Kuparuk Topset & 0.5 & 0.5 & 0.9 & 0.9 & 0.9 & & & & & & & & & & & & & & & & & & \\
\hline 7 & Beaufortian Cretaceous Shelf Margin & 0.1 & 0.1 & 0.5 & 0.5 & 0.9 & 0.5 & & & & & & & & & & & & & & & & & \\
\hline 8 & Triassic Barrow Arch & 0.1 & 0.1 & 0.1 & 0.1 & 0.1 & 0.5 & 0.1 & & & & & & & & & & & & & & & & \\
\hline 9 & Ivishak Barrow Flank & 0.1 & 0.1 & 0.1 & 0.1 & 0.1 & 0.5 & 0.1 & 0.1 & & & & & & & & & & & & & & & \\
\hline 10 & Endicott & 0.1 & 0.1 & 0.1 & 0.1 & 0.1 & 0.1 & 0.1 & 0.1 & 0.5 & & & & & & & & & & & & & & \\
\hline 11 & Endicott Truncation & 0.5 & 0.1 & 0.1 & 0.1 & 0.1 & 0.1 & 0.1 & 0.9 & 0.1 & 0.1 & & & & & & & & & & & & & \\
\hline 12 & Franklinian & 0.1 & 0.1 & 0.1 & 0.1 & 0.1 & 0.1 & 0.1 & 0.9 & 0.1 & 0.1 & 0.9 & & & & & & & & & & & & \\
\hline 13 & Lisburne Barrow Arch & 0.1 & 0.1 & 0.1 & 0.1 & 0.1 & 0.1 & 0.1 & 0.9 & 0.1 & 0.1 & 0.9 & 0.9 & & & & & & & & & & & \\
\hline 14 & Lisburne Barrow Flank & 0.1 & 0.1 & 0.1 & 0.1 & 0.1 & 0.1 & 0.1 & 0.1 & 0.5 & 0.5 & 0.5 & 0.1 & 0.5 & & & & & & & & & & \\
\hline 15 & Kemik-Thompson & 0.5 & 0.1 & 0.1 & 0.1 & 0.1 & 0.5 & 0.1 & 0.9 & 0.1 & 0.1 & 0.9 & 0.9 & 0.9 & 0.1 & & & & & & & & & \\
\hline 16 & Basement Involved Structural & 0.1 & 0.1 & 0.5 & 0.1 & 0.1 & 0.5 & 0.5 & 0.1 & 0.5 & 0.5 & 0.5 & 0.5 & 0.1 & 0.5 & 0.5 & & & & & & & & \\
\hline 17 & Beaufortian Structural & 0.5 & 0.1 & 0.5 & 0.1 & 0.5 & 0.5 & 0.9 & 0.1 & 0.5 & 0.1 & 0.1 & 0.1 & 0.1 & 0.1 & 0.5 & 0.5 & & & & & & & \\
\hline 18 & Brookian Clinoform Structural South & 0.5 & 0.5 & 0.1 & 0.1 & 0.1 & 0.1 & 0.5 & 0.1 & 0.1 & 0.1 & 0.1 & 0.1 & 0.1 & 0.1 & 0.1 & 0.1 & 0.9 & & & & & & \\
\hline 19 & Brookian Clinoform Structural North & 0.9 & 0.9 & 0.1 & 0.1 & 0.1 & 0.1 & 0.1 & 0.1 & 0.1 & 0.1 & 0.1 & 0.1 & 0.1 & 0.1 & 0.5 & 0.1 & 0.9 & 0.5 & & & & & \\
\hline 20 & Brookian Topset Structural South & 0.5 & 0.5 & 0.1 & 0.1 & 0.1 & 0.1 & 0.5 & 0.1 & 0.1 & 0.1 & 0.1 & 0.1 & 0.1 & 0.1 & 0.1 & 0.1 & 0.1 & 0.9 & 0.5 & & & & \\
\hline 21 & Brookian Topset Structural North & 0.9 & 0.9 & 0.1 & 0.1 & 0.1 & 0.1 & 0.1 & 0.1 & 0.1 & 0.1 & 0.1 & 0.1 & 0.1 & 0.1 & 0.1 & 0.1 & 0.1 & 0.5 & 0.9 & 0.5 & & & \\
\hline 22 & Thrust Belt Triangle Zone & 0.1 & 0.1 & 0.1 & 0.1 & 0.1 & 0.1 & 0.1 & 0.1 & 0.1 & 0.1 & 0.1 & 0.1 & 0.1 & 0.1 & 0.1 & 0.5 & 0.5 & 0.9 & 0.1 & 0.5 & 0.1 & & \\
\hline 23 & Thrust Belt Lisburne & 0.1 & 0.1 & 0.1 & 0.1 & 0.1 & 0.1 & 0.1 & 0.1 & 0.1 & 0.1 & 0.1 & 0.1 & 0.1 & 0.1 & 0.1 & 0.1 & 0.1 & 0.5 & 0.1 & 0.5 & 0.1 & 0.9 & \\
\hline 24 & Ellesmerian Structural & 0.1 & 0.1 & 0.1 & 0.1 & 0.1 & 0.1 & 0.1 & 0.1 & 0.5 & 0.5 & 0.5 & 0.5 & 0.1 & 0.5 & 0.5 & 0.9 & 0.5 & 0.1 & 0.1 & 0.1 & 0.1 & 0.5 & 0.1 \\
\hline
\end{tabular}


Table $5 \mathrm{~b}$. Assessor specified Trap dependencies between plays

[Correlations: 0.1 , low; 0.5, medium; 0.9 , high]

\begin{tabular}{|c|c|c|c|c|c|c|c|c|c|c|c|c|c|c|c|c|c|c|c|c|c|c|c|c|}
\hline & Trap & 1 & 2 & 3 & 4 & 5 & 6 & 7 & 8 & 9 & 10 & 11 & 12 & 13 & 14 & 15 & 16 & 17 & 18 & 19 & 20 & 21 & 22 & 23 \\
\hline & Brookian Clinoform & & & & & & & & & & & & & & & & & & & & & & & \\
\hline 2 & Brookian Topset & 0.1 & & & & & & & & & & & & & & & & & & & & & & \\
\hline 3 & Beaufortian Upper Jurassic Topset East & 0.1 & 0.1 & & & & & & & & & & & & & & & & & & & & & \\
\hline 4 & Beaufortian Upper Jurassic Topset West & 0.1 & 0.1 & 0.5 & & & & & & & & & & & & & & & & & & & & \\
\hline 5 & Beaufortian Clinoform & 0.1 & 0.1 & 0.1 & 0.1 & & & & & & & & & & & & & & & & & & & \\
\hline 6 & Beaufortian Kuparuk Topset & 0.1 & 0.1 & 0.1 & 0.1 & 0.1 & & & & & & & & & & & & & & & & & & \\
\hline 7 & Beaufortian Cretaceous Shelf Margin & 0.1 & 0.1 & 0.1 & 0.1 & 0.1 & 0.1 & & & & & & & & & & & & & & & & & \\
\hline 8 & Triassic Barrow Arch & 0.1 & 0.1 & 0.1 & 0.1 & 0.1 & 0.1 & 0.1 & & & & & & & & & & & & & & & & \\
\hline 9 & Ivishak Barrow Flank & 0.1 & 0.1 & 0.1 & 0.1 & 0.1 & 0.1 & 0.1 & 0.1 & & & & & & & & & & & & & & & \\
\hline 10 & Endicott & 0.1 & 0.1 & 0.1 & 0.1 & 0.1 & 0.1 & 0.1 & 0.1 & 0.1 & & & & & & & & & & & & & & \\
\hline 11 & Endicott Truncation & 0.1 & 0.1 & 0.1 & 0.1 & 0.1 & 0.1 & 0.1 & 0.5 & 0.1 & 0.1 & & & & & & & & & & & & & \\
\hline 12 & Franklinian & 0.1 & 0.1 & 0.1 & 0.1 & 0.1 & 0.1 & 0.1 & 0.1 & 0.1 & 0.1 & 0.1 & & & & & & & & & & & & \\
\hline 13 & Lisburne Barrow Arch & 0.1 & 0.1 & 0.1 & 0.1 & 0.1 & 0.1 & 0.1 & 0.5 & 0.1 & 0.1 & 0.5 & 0.1 & & & & & & & & & & & \\
\hline 14 & Lisburne Barrow Flank & 0.1 & 0.1 & 0.1 & 0.1 & 0.1 & 0.1 & 0.1 & 0.1 & 0.1 & 0.1 & 0.1 & 0.1 & 0.1 & & & & & & & & & & \\
\hline 15 & Kemik-Thompson & 0.1 & 0.1 & 0.1 & 0.1 & 0.1 & 0.1 & 0.1 & 0.1 & 0.1 & 0.1 & 0.1 & 0.1 & 0.1 & 0.1 & & & & & & & & & \\
\hline 16 & Basement Involved Structural & 0.1 & 0.1 & 0.1 & 0.1 & 0.1 & 0.1 & 0.1 & 0.1 & 0.5 & 0.1 & 0.1 & 0.1 & 0.1 & 0.5 & 0.1 & & & & & & & & \\
\hline 17 & Beaufortian Structural & 0.1 & 0.1 & 0.1 & 0.1 & 0.1 & 0.1 & 0.5 & 0.1 & 0.1 & 0.1 & 0.1 & 0.1 & 0.1 & 0.1 & 0.5 & 0.1 & & & & & & & \\
\hline 18 & Brookian Clinoform Structural South & 0.5 & 0.1 & 0.1 & 0.1 & 0.1 & 0.1 & 0.1 & 0.1 & 0.1 & 0.1 & 0.1 & 0.1 & 0.1 & 0.1 & 0.1 & 0.1 & 0.1 & & & & & & \\
\hline 19 & Brookian Clinoform Structural North & 0.5 & 0.1 & 0.1 & 0.1 & 0.1 & 0.1 & 0.1 & 0.1 & 0.1 & 0.1 & 0.1 & 0.1 & 0.1 & 0.1 & 0.1 & 0.1 & 0.1 & 0.9 & & & & & \\
\hline 20 & Brookian Topset Structural South & 0.1 & 0.5 & 0.1 & 0.1 & 0.1 & 0.1 & 0.1 & 0.1 & 0.1 & 0.1 & 0.1 & 0.1 & 0.1 & 0.1 & 0.1 & 0.1 & 0.1 & 0.1 & 0.1 & & & & \\
\hline 21 & Brookian Topset Structural North & 0.1 & 0.5 & 0.1 & 0.1 & 0.1 & 0.1 & 0.1 & 0.1 & 0.1 & 0.1 & 0.1 & 0.1 & 0.1 & 0.1 & 0.1 & 0.1 & 0.1 & 0.1 & 0.1 & 0.9 & & & \\
\hline 22 & Thrust Belt Triangle Zone & 0.1 & 0.1 & 0.1 & 0.1 & 0.1 & 0.1 & 0.1 & 0.1 & 0.1 & 0.1 & 0.1 & 0.1 & 0.1 & 0.1 & 0.1 & 0.1 & 0.1 & 0.9 & 0.5 & 0.1 & 0.1 & & \\
\hline 23 & Thrust Belt Lisburne & 0.1 & 0.1 & 0.1 & 0.1 & 0.1 & 0.1 & 0.1 & 0.1 & 0.1 & 0.1 & 0.1 & 0.1 & 0.1 & 0.5 & 0.1 & 0.1 & 0.1 & 0.1 & 0.1 & 0.1 & 0.1 & 0.5 & \\
\hline 24 & Ellesmerian structural & 0.1 & 0.1 & 0.1 & 0.1 & 0.1 & 0.1 & 0.1 & 0.1 & 0.5 & 0.1 & 0.1 & 0.1 & 0.1 & 0.5 & 0.1 & 0.9 & 0.1 & 0.1 & 0.1 & 0.1 & 0.1 & 0.1 & 0.5 \\
\hline
\end{tabular}


Table $5 \mathrm{c}$. Assessor specified Timing dependencies between plays.

[Correlations: 0.1, low; 0.5, medium; 0.9, high]

\begin{tabular}{|c|c|c|c|c|c|c|c|c|c|c|c|c|c|c|c|c|c|c|c|c|c|c|c|}
\hline & 1 & 2 & 3 & 4 & 5 & 6 & 7 & 8 & 9 & 10 & 11 & 12 & 13 & 14 & 15 & 16 & 17 & 18 & 19 & 20 & 21 & 22 & 23 \\
\hline 1 Brookian Clinoform & & & & & & & & & & & & & & & & & & & & & & & \\
\hline 2 Brookian Topset & 0.1 & & & & & & & & & & & & & & & & & & & & & & \\
\hline 3 Beaufortian Upper Jurassic Topset East & 0.1 & 0.1 & & & & & & & & & & & & & & & & & & & & & \\
\hline 4 Beaufortian Upper Jurassic Topset West & 0.1 & 0.1 & 0.1 & & & & & & & & & & & & & & & & & & & & \\
\hline 5 Beaufortian Clinoform & 0.1 & 0.1 & 0.1 & 0.1 & & & & & & & & & & & & & & & & & & & \\
\hline 6 Beaufortian Kuparuk Topset & 0.1 & 0.1 & 0.1 & 0.1 & 0.1 & & & & & & & & & & & & & & & & & & \\
\hline 7 Beaufortian Cretaceous Shelf Margin & 0.1 & 0.1 & 0.1 & 0.1 & 0.1 & 0.1 & & & & & & & & & & & & & & & & & \\
\hline 8 Triassic Barrow Arch & 0.1 & 0.1 & 0.1 & 0.1 & 0.1 & 0.1 & 0.1 & & & & & & & & & & & & & & & & \\
\hline 9 Ivishak Barrow Flank & 0.1 & 0.1 & 0.1 & 0.1 & 0.1 & 0.1 & 0.1 & 0.1 & & & & & & & & & & & & & & & \\
\hline 10 Endicott & 0.1 & 0.1 & 0.1 & 0.1 & 0.1 & 0.1 & 0.1 & 0.1 & 0.1 & & & & & & & & & & & & & & \\
\hline 11 Endicott Truncation & 0.1 & 0.1 & 0.1 & 0.1 & 0.1 & 0.1 & 0.1 & 0.5 & 0.1 & 0.1 & & & & & & & & & & & & & \\
\hline 12 Franklinian & 0.1 & 0.1 & 0.1 & 0.1 & 0.1 & 0.1 & 0.1 & 0.5 & 0.1 & 0.1 & 0.5 & & & & & & & & & & & & \\
\hline 13 Lisburne Barrow Arch & 0.1 & 0.1 & 0.1 & 0.1 & 0.1 & 0.1 & 0.1 & 0.5 & 0.1 & 0.1 & 0.5 & 0.5 & & & & & & & & & & & \\
\hline 14 Lisburne Barrow Flank & 0.1 & 0.1 & 0.1 & 0.1 & 0.1 & 0.1 & 0.1 & 0.1 & 0.1 & 0.1 & 0.1 & 0.1 & 0.1 & & & & & & & & & & \\
\hline 15 Kemik-Thompson & 0.1 & 0.1 & 0.1 & 0.1 & 0.1 & 0.1 & 0.1 & 0.1 & 0.1 & 0.1 & 0.5 & 0.1 & 0.1 & 0.1 & & & & & & & & & \\
\hline 16 Basement Involved Structural & 0.1 & 0.1 & 0.1 & 0.1 & 0.1 & 0.1 & 0.1 & 0.1 & 0.1 & 0.1 & 0.1 & 0.1 & 0.1 & 0.1 & 0.1 & & & & & & & & \\
\hline 17 Beaufortian Structural & 0.1 & 0.1 & 0.1 & 0.1 & 0.1 & 0.1 & 0.1 & 0.1 & 0.1 & 0.1 & 0.1 & 0.1 & 0.1 & 0.1 & 0.1 & 0.1 & & & & & & & \\
\hline 18 Brookian Clinoform Structural South & 0.1 & 0.1 & 0.1 & 0.1 & 0.1 & 0.1 & 0.1 & 0.1 & 0.1 & 0.1 & 0.1 & 0.1 & 0.1 & 0.1 & 0.1 & 0.1 & 0.5 & & & & & & \\
\hline 19 Brookian Clinoform Structural North & 0.1 & 0.1 & 0.1 & 0.1 & 0.1 & 0.1 & 0.1 & 0.1 & 0.1 & 0.1 & 0.1 & 0.1 & 0.1 & 0.1 & 0.1 & 0.1 & 0.5 & 0.5 & & & & & \\
\hline 20 Brookian Topset Structural South & 0.1 & 0.1 & 0.1 & 0.1 & 0.1 & 0.1 & 0.1 & 0.1 & 0.1 & 0.1 & 0.1 & 0.1 & 0.1 & 0.1 & 0.1 & 0.1 & 0.5 & 0.9 & 0.5 & & & & \\
\hline 21 Brookian Topset Structural North & 0.1 & 0.1 & 0.1 & 0.1 & 0.1 & 0.1 & 0.1 & 0.1 & 0.1 & 0.1 & 0.1 & 0.1 & 0.1 & 0.1 & 0.1 & 0.1 & 0.5 & 0.5 & 0.9 & 0.5 & & & \\
\hline 22 Thrust Belt Triangle Zone & 0.1 & 0.1 & 0.1 & 0.1 & 0.1 & 0.1 & 0.1 & 0.1 & 0.1 & 0.1 & 0.1 & 0.1 & 0.1 & 0.1 & 0.1 & 0.1 & 0.5 & 0.9 & 0.1 & 0.9 & 0.1 & & \\
\hline 23 Thrust Belt Lisburne & 0.1 & 0.1 & 0.1 & 0.1 & 0.1 & 0.1 & 0.1 & 0.1 & 0.1 & 0.1 & 0.1 & 0.1 & 0.1 & 0.1 & 0.1 & 0.1 & 0.1 & 0.5 & 0.1 & 0.5 & 0.1 & 0.9 & \\
\hline 24 Ellesmerian structural & 0.1 & 0.1 & 0.1 & 0.1 & 0.1 & 0.1 & 0.1 & 0.1 & 0.1 & 0.1 & 0.1 & 0.1 & 0.1 & 0.1 & 0.1 & 0.9 & 0.1 & 0.1 & 0.1 & 0.1 & 0.1 & 0.1 & \\
\hline
\end{tabular}


Table 5d. Arithmetic average of assessor specified Charge, Trap, and Timing correlatiions.

\begin{tabular}{|c|c|c|c|c|c|c|c|c|c|c|c|c|c|c|c|c|c|c|c|c|c|c|c|}
\hline & 1 & 2 & 3 & 4 & 5 & 6 & 7 & 8 & 9 & 10 & 11 & 12 & 13 & 14 & 15 & 16 & 17 & 18 & 19 & 20 & 21 & 22 & 23 \\
\hline 1 Brookian Clinoform & & & & & & & & & & & & & & & & & & & & & & & \\
\hline 2 Brookian Topset & 0.37 & & & & & & & & & & & & & & & & & & & & & & \\
\hline 3 Beaufortian Upper Jurassic Topset East & 0.10 & 0.10 & & & & & & & & & & & & & & & & & & & & & \\
\hline 4 Beaufortian Upper Jurassic Topset West & 0.10 & 0.10 & 0.50 & & & & & & & & & & & & & & & & & & & & \\
\hline 5 Beaufortian Clinoform & 0.10 & 0.10 & 0.37 & 0.37 & & & & & & & & & & & & & & & & & & & \\
\hline 6 Beaufortian Kuparuk Topset & 0.23 & 0.23 & 0.37 & 0.37 & 0.37 & & & & & & & & & & & & & & & & & & \\
\hline 7 Beaufortian Cretaceous Shelf Margin & 0.10 & 0.10 & 0.23 & 0.23 & 0.37 & 0.23 & & & & & & & & & & & & & & & & & \\
\hline 8 Triassic Barrow Arch & 0.10 & 0.10 & 0.10 & 0.10 & 0.10 & 0.23 & 0.10 & & & & & & & & & & & & & & & & \\
\hline 9 Ivishak Barrow Flank & 0.10 & 0.10 & 0.10 & 0.10 & 0.10 & 0.23 & 0.10 & 0.10 & & & & & & & & & & & & & & & \\
\hline 10 Endicott & 0.10 & 0.10 & 0.10 & 0.10 & 0.10 & 0.10 & 0.10 & 0.10 & 0.23 & & & & & & & & & & & & & & \\
\hline 11 Endicott Truncation & 0.23 & 0.10 & 0.10 & 0.10 & 0.10 & 0.10 & 0.10 & 0.63 & 0.10 & 0.10 & & & & & & & & & & & & & \\
\hline 12 Franklinian & 0.10 & 0.10 & 0.10 & 0.10 & 0.10 & 0.10 & 0.10 & 0.50 & 0.10 & 0.10 & 0.50 & & & & & & & & & & & & \\
\hline 13 Lisburne Barrow Arch & 0.10 & 0.10 & 0.10 & 0.10 & 0.10 & 0.10 & 0.10 & 0.63 & 0.10 & 0.10 & 0.63 & 0.50 & & & & & & & & & & & \\
\hline 14 Lisburne Barrow Flank & 0.10 & 0.10 & 0.10 & 0.10 & 0.10 & 0.10 & 0.10 & 0.10 & 0.23 & 0.23 & 0.23 & 0.10 & 0.23 & & & & & & & & & & \\
\hline 15 Kemik-Thompson & 0.23 & 0.10 & 0.10 & 0.10 & 0.10 & 0.23 & 0.10 & 0.37 & 0.10 & 0.10 & 0.50 & 0.37 & 0.37 & 0.10 & & & & & & & & & \\
\hline 16 Basement Involved Structural & 0.10 & 0.10 & 0.23 & 0.10 & 0.10 & 0.23 & 0.23 & 0.10 & 0.37 & 0.23 & 0.23 & 0.23 & 0.10 & 0.37 & 0.23 & & & & & & & & \\
\hline 17 Beaufortian Structural & 0.23 & 0.10 & 0.23 & 0.10 & 0.23 & 0.23 & 0.50 & 0.10 & 0.23 & 0.10 & 0.10 & 0.10 & 0.10 & 0.10 & 0.37 & 0.23 & & & & & & & \\
\hline 18 Brookian Clinoform Structural South & 0.37 & 0.23 & 0.10 & 0.10 & 0.10 & 0.10 & 0.23 & 0.10 & 0.10 & 0.10 & 0.10 & 0.10 & 0.10 & 0.10 & 0.10 & 0.10 & 0.50 & & & & & & \\
\hline 19 Brookian Clinoform Structural North & 0.50 & 0.37 & 0.10 & 0.10 & 0.10 & 0.10 & 0.10 & 0.10 & 0.10 & 0.10 & 0.10 & 0.10 & 0.10 & 0.10 & 0.23 & 0.10 & 0.50 & 0.63 & & & & & \\
\hline 20 Brookian Topset Structural South & 0.23 & 0.37 & 0.10 & 0.10 & 0.10 & 0.10 & 0.23 & 0.10 & 0.10 & 0.10 & 0.10 & 0.10 & 0.10 & 0.10 & 0.10 & 0.10 & 0.23 & 0.63 & 0.37 & & & & \\
\hline 21 Brookian Topset Structural North & 0.37 & 0.50 & 0.10 & 0.10 & 0.10 & 0.10 & 0.10 & 0.10 & 0.10 & 0.10 & 0.10 & 0.10 & 0.10 & 0.10 & 0.10 & 0.10 & 0.23 & 0.37 & 0.63 & 0.63 & & & \\
\hline 22 Thrust Belt Triangle Zone & 0.10 & 0.10 & 0.10 & 0.10 & 0.10 & 0.10 & 0.10 & 0.10 & 0.10 & 0.10 & 0.10 & 0.10 & 0.10 & 0.10 & 0.10 & 0.23 & 0.37 & 0.90 & 0.23 & 0.50 & 0.10 & & \\
\hline 23 Thrust Belt Lisburne & 0.10 & 0.10 & 0.10 & 0.10 & 0.10 & 0.10 & 0.10 & 0.10 & 0.10 & 0.10 & 0.10 & 0.10 & 0.10 & 0.23 & 0.10 & 0.10 & 0.10 & 0.37 & 0.10 & 0.37 & 0.10 & 0.77 & \\
\hline 24 Ellesmerian structural & 0.10 & 0.10 & 0.10 & 0.10 & 0.10 & 0.10 & 0.10 & 0.10 & 0.37 & 0.23 & 0.23 & 0.23 & 0.10 & 0.37 & 0.23 & 0.90 & 0.23 & 0.10 & 0.10 & 0.10 & 0.10 & 0.23 & 0.23 \\
\hline
\end{tabular}


Table 5e. Adjusted dependency matrix.

\begin{tabular}{|c|c|c|c|c|c|c|c|c|c|c|c|c|c|c|c|c|c|c|c|c|c|c|c|c|}
\hline & & 1 & 2 & 3 & 4 & 5 & 6 & 7 & 8 & 9 & 10 & 11 & 12 & 13 & 14 & 15 & 16 & 17 & 18 & 19 & 20 & 21 & 22 & 23 \\
\hline 1 & Brookian Clinoform & & & & & & & & & & & & & & & & & & & & & & & \\
\hline 2 & Brookian Topset & 0.33 & & & & & & & & & & & & & & & & & & & & & & \\
\hline 3 & Beaufortian Upper Jurassic Topset East & 0.09 & 0.09 & & & & & & & & & & & & & & & & & & & & & \\
\hline 4 & Beaufortian Upper Jurassic Topset West & 0.09 & 0.09 & 0.45 & & & & & & & & & & & & & & & & & & & & \\
\hline 5 & Beaufortian Clinoform & 0.09 & 0.09 & 0.33 & 0.33 & & & & & & & & & & & & & & & & & & & \\
\hline 6 & Beaufortian Kuparuk Topset & 0.21 & 0.21 & 0.33 & 0.33 & 0.33 & & & & & & & & & & & & & & & & & & \\
\hline 7 & Beaufortian Cretaceous Shelf Margin & 0.09 & 0.09 & 0.21 & 0.21 & 0.33 & 0.21 & & & & & & & & & & & & & & & & & \\
\hline 8 & Triassic Barrow Arch & 0.09 & 0.09 & 0.09 & 0.09 & 0.09 & 0.21 & 0.09 & & & & & & & & & & & & & & & & \\
\hline 9 & Ivishak Barrow Flank & 0.09 & 0.09 & 0.09 & 0.09 & 0.09 & 0.21 & 0.09 & 0.09 & & & & & & & & & & & & & & & \\
\hline 0 & Endicott & 0.09 & 0.09 & 0.09 & 0.09 & 0.09 & 0.09 & 0.09 & 0.09 & 0.21 & & & & & & & & & & & & & & \\
\hline 1 & Endicott Truncation & 0.21 & 0.09 & 0.09 & 0.09 & 0.09 & 0.09 & 0.09 & 0.57 & 0.09 & 0.09 & & & & & & & & & & & & & \\
\hline 2 & Franklinian & 0.09 & 0.09 & 0.09 & 0.09 & 0.09 & 0.09 & 0.09 & 0.45 & 0.09 & 0.09 & 0.45 & & & & & & & & & & & & \\
\hline 3 & Lisburne Barrow Arch & 0.09 & 0.09 & 0.09 & 0.09 & 0.09 & 0.09 & 0.09 & 0.57 & 0.09 & 0.09 & 0.57 & 0.45 & & & & & & & & & & & \\
\hline 4 & Lisburne Barrow Flank & 0.09 & 0.09 & 0.09 & 0.09 & 0.09 & 0.09 & 0.09 & 0.09 & 0.21 & 0.21 & 0.21 & 0.09 & 0.21 & & & & & & & & & & \\
\hline 5 & Kemik-Thompson & 0.21 & 0.09 & 0.09 & 0.09 & 0.09 & 0.21 & 0.09 & 0.33 & 0.09 & 0.09 & 0.45 & 0.33 & 0.33 & 0.09 & & & & & & & & & \\
\hline 6 & Basement Involved Structural & 0.09 & 0.09 & 0.21 & 0.09 & 0.09 & 0.21 & 0.21 & 0.09 & 0.33 & 0.21 & 0.21 & 0.21 & 0.09 & 0.33 & 0.21 & & & & & & & & \\
\hline 7 & Beaufortian Structural & 0.21 & 0.09 & 0.21 & 0.09 & 0.21 & 0.21 & 0.45 & 0.09 & 0.21 & 0.09 & 0.09 & 0.09 & 0.09 & 0.09 & 0.33 & 0.21 & & & & & & & \\
\hline 8 & Brookian Clinoform Structural South & 0.33 & 0.21 & 0.09 & 0.09 & 0.09 & 0.09 & 0.21 & 0.09 & 0.09 & 0.09 & 0.09 & 0.09 & 0.09 & 0.09 & 0.09 & 0.09 & 0.45 & & & & & & \\
\hline 9 & Brookian Clinoform Structural North & 0.45 & 0.33 & 0.09 & 0.09 & 0.09 & 0.09 & 0.09 & 0.09 & 0.09 & 0.09 & 0.09 & 0.09 & 0.09 & 0.09 & 0.21 & 0.09 & 0.45 & 0.57 & & & & & \\
\hline 0 & Brookian Topset Structural South & 0.21 & 0.33 & 0.09 & 0.09 & 0.09 & 0.09 & 0.21 & 0.09 & 0.09 & 0.09 & 0.09 & 0.09 & 0.09 & 0.09 & 0.09 & 0.09 & 0.21 & 0.57 & 0.33 & & & & \\
\hline 1 & Brookian Topset Structural North & 0.33 & 0.45 & 0.09 & 0.09 & 0.09 & 0.09 & 0.09 & 0.09 & 0.09 & 0.09 & 0.09 & 0.09 & 0.09 & 0.09 & 0.09 & 0.09 & 0.21 & 0.33 & 0.57 & 0.57 & & & \\
\hline 2 & Thrust Belt Triangle Zone & 0.09 & 0.09 & 0.09 & 0.09 & 0.09 & 0.09 & 0.09 & 0.09 & 0.09 & 0.09 & 0.09 & 0.09 & 0.09 & 0.09 & 0.09 & 0.21 & 0.33 & 0.81 & 0.21 & 0.45 & 0.09 & & \\
\hline 3 & Thrust Belt Lisburne & 0.09 & 0.09 & 0.09 & 0.09 & 0.09 & 0.09 & 0.09 & 0.09 & 0.09 & 0.09 & 0.09 & 0.09 & 0.09 & 0.21 & 0.09 & 0.09 & 0.09 & 0.33 & 0.09 & 0.33 & 0.09 & 0.69 & \\
\hline 24 & Ellesmerian structural & 0.09 & 0.09 & 0.09 & 0.09 & 0.09 & 0.09 & 0.09 & 0.09 & 0.33 & 0.21 & 0.21 & 0.21 & 0.09 & 0.33 & 0.21 & 0.81 & 0.21 & 0.09 & 0.09 & 0.09 & 0.09 & 0.21 & 0.21 \\
\hline
\end{tabular}


range of assessor estimated uncertainty. The resultant correlation matrix (table 5e) was used for the remaining part of the analysis. This aggregation procedure is similar to that used in the most recent USGS NPRA assessment (Schuenemeyer, 2003). It is also virtually identical to the dependency scheme used in the USGS 1995 National Assessment (Gautier and other, 1995).

\section{Generating a Correlated Sample}

The adjusted correlation matrix (table 5e) was then used to induce the appropriate correlation structure in the data. A justification for this procedure was given previously. The algorithm is essentially the same as that used to generate correlated samples of accumulation attributes. The procedure is outlined below.

1. Let $\mathrm{R}$ be the $24 \times 24$ adjusted (permissible) correlation matrix of play dependencies. Also, let $\mathrm{ns}=10,000$, where $\mathrm{ns}$ is the number of simulation runs.

2. Perform a Cholesky factorization on $\mathrm{R}$ to obtain a lower triangular matrix plus the diagonal matrix, call this $A$, such that $A A^{\prime}=R$ (where $A^{\prime}$ is the transpose of $A$ ).

3. Let $\mathrm{Ua}$ be an ns $\mathrm{x} 24$ matrix, where each row of $\mathrm{Ua}$ is an independent set of continuous uniform random numbers between -1 and 1 .

4. Let $\mathrm{Uac}=\mathrm{Ua} \times \mathrm{A}$.

5. Let $\operatorname{Ur}[, \mathrm{i}]=\operatorname{Rank}(\operatorname{Uac}[, \mathrm{i}]), \mathrm{i}=1, \ldots, 24$.

6. Adjust the matrix Ur by the total number of plays run (10,000/play probability).

7. The ranked values in the columns of Ur then represent the sample numbers of the play (with play recoverable barrels of oil equivalent sorted in ascending order) necessary to achieve the desired correlation. (The matrix Ur in the context of the Central North Slope assessment is file AMrand.)

Each element in the matrix Ur became a sample number. As previously discussed, only the expected number of successful plays was generated; however, sample numbers from unsuccessful plays were needed to generate samples for the aggregate distributions. For example, as previously noted, the Beaufortian Clinoform has 16,667 expected number of runs, however, only 10,000 simulation runs were made. In sampling for aggregation, we take a simple random sample of 10,000 from a population of 16,667 runs, 6,667 of which were a priori unsuccessful. For those plays that consisted of 10,000 runs (i.e., the play favorability probability was 1.0), such as the Brookian Topset, this procedure generated a permutation of the original data that imparted the appropriate correlation structure. A rank correlation structure was used because the oil and gas distributions differ widely among the 24 plays. The standard (Pearson) correlation coefficient is only meaningful when distributions are similar and in particular when they are symmetric. The sample numbers to achieve the desired correlation structure were generated by function AMcorr.fn.

The actual process of aggregation, performed by algorithm AMagg.fn, was straightforward. Samples were selected by row from matrix Ur and the corresponding values of oil or gas were obtained from the appropriate play and/or prospect file. There 
were 10,000 simulation runs in each of the play/prospect files. The unsuccessful runs resulting from a favorable play probability less than one were assumed to follow the actual 10,000 runs generated from the simulation for purposes of sampling. The output file (file AMag) contains aggregate results (one line for each of the 10,000 simulations) plus sample numbers for each play that reference the run numbers in the simulation and play files. A part of this file is given in Table 6.

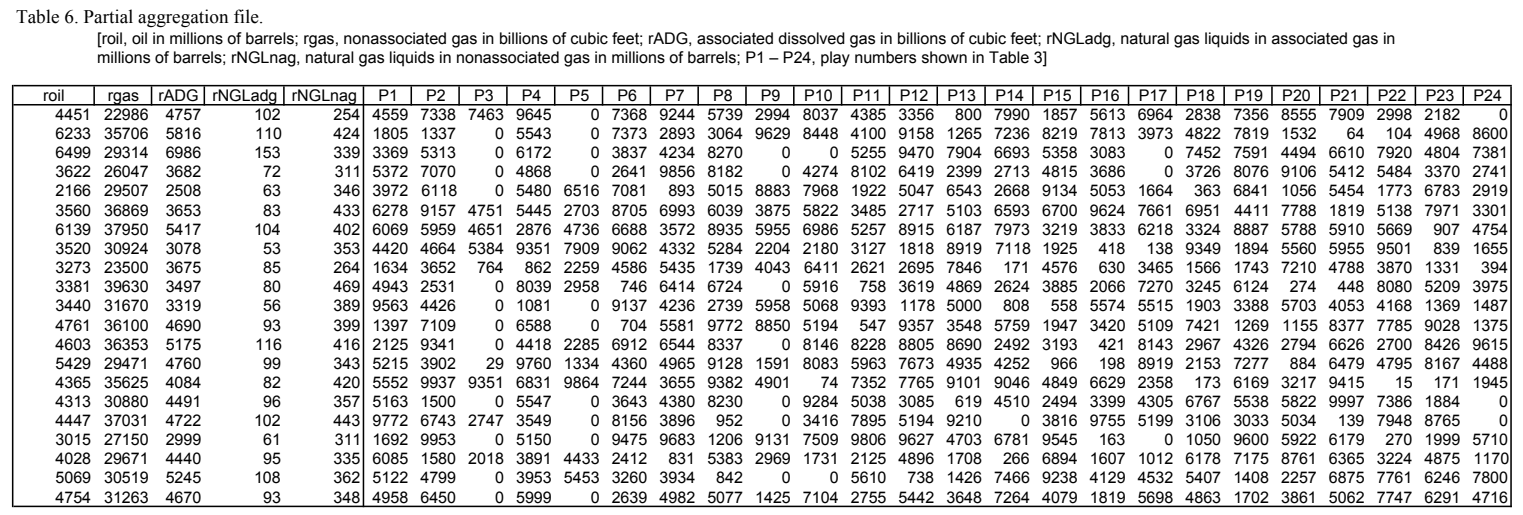

The estimates of uncertainty at the aggregate level were performed by function AMsummary.fn from the merged results. Summary results included the mean, standard deviation, and F95, F50, and F05 fractiles for recoverable oil and nonassociated gas (table 7).

Table 7. Summary statistics for distributions of technically recoverable undiscovered oil, nonassociated gas and derivatives for the central North Slope assessment area.

[MMBBL, million barrels; BCF, billion cubic feet; NGL, natural gas liquids]

\begin{tabular}{|l|r|r|r|r|r|}
\hline Statistic & $\begin{array}{c}\text { Oil } \\
\text { (MMBL) }\end{array}$ & $\begin{array}{c}\text { Nonassociated } \\
\text { Gas (BCF) }\end{array}$ & $\begin{array}{c}\text { Associated } \\
\text { Dissolved } \\
\text { Gas (BCF) }\end{array}$ & $\begin{array}{c}\text { NGL } \\
\text { Associated } \\
\text { Gas (MMBBL) }\end{array}$ & $\begin{array}{c}\text { NGL } \\
\text { Nonassociated } \\
\text { Gas (MMBB) }\end{array}$ \\
\hline Min & 1567 & 16392 & 1539 & 26 & 187 \\
\hline F95 & 2565 & 23939 & 2681 & 55 & 278 \\
\hline F75 & 3249 & 28806 & 3444 & 73 & 334 \\
\hline F50 & 3851 & 32678 & 4080 & 88 & 380 \\
\hline F25 & 4580 & 37059 & 4844 & 107 & 431 \\
\hline F05 & 5854 & 44873 & 6092 & 138 & 521 \\
\hline Max & 10220 & 71216 & 9770 & 231 & 817 \\
\hline Mean & 3984 & 33318 & 4198 & 91 & 387 \\
\hline Std Dev & 1014 & 6471 & 1051 & 26 & 75 \\
\hline N & 10000 & 10000 & 10000 & 10000 & 10000 \\
\hline
\end{tabular}

For purposes of economic analysis it was desired to obtain an estimate of the field size distributions at each of the fractiles. To accomplish this and reduce sensitivity to the choice of a single fractile, the actual observation at the F95, F50, and F05 fractiles plus 10 observations on either side of this value were extracted and provide USGS economist Emil Attanasi, with data for economic analysis (file AMag). Note that the averages of the 
fields in the 24 simulated plays bracketing the fractiles will only be approximately equal to the estimates of the fractiles provided in the summary statistics (file AMss).

\section{References}

Amyx, J.W., D.M. Bass, Jr., and R.L. Whiting, 1960, Petroleum Reservoir Engineering, McGraw-Hill, 610 p.

Bird, K.J., and Houseknecht, D.W., 2002, U.S. Geological Survey 2002 petroleum resource assessment of the National Petroleum Reserve in Alaska (NPRA): USGS Fact Sheet 045-02, 6 p. Also available online at: http://geopubs.wr.usgs.gov/fact-sheet/fs045-02/

Bird, K.J., Houseknecht, D.W., Attanasi, E.D., Moore, T.E., Nelson, P.H., Potter, C.J., Schenk, C.J., Schuenemeyer, J.H., Verma, M.K., Saltus, R.W., Phillips, J.D., Charpentier, R.R., Cook, T.A., Klett, T.R., Pollastro, R.M., 2005, Oil and gas assessment of central North Slope, Alaska, 2005: U. S. Geological Survey Fact Sheet 20053043 (available online at http://pubs.usgs.gov/fs/2005/3043/), p. 2.

Garrity, C.P., Houseknecht, D.W., Bird, K.J., Moore, T.E., Potter, C.J., Nelson, P.H., Schenk, C.J., 2005, U.S. Geological Survey 2005 oil and gas resource assessment of the central North Slope, Alaska: Play maps and results: U. S. Geological Survey Open-File Report 2005-1182 (available on-line at http://pubs.usgs.gov/of/2005/1182/, p. 29.

Gautier, D.L, Dolton, G.L., Takahashi, L.I., and Varnes, K.L., eds., 1995, 1995 National Assessment of the United States Oil and Gas Resources-Results, Methodology, and Supporting Data, U.S. Geological Survey Digital Data Series DDS-30.

Kotz, S., Balakrishnan, N., and Johnson, N.L., 2000, Continuous Multivariate Distributions Volume 1: Models and Applications, $2^{\text {nd }}$ Ed., Wiley. New York.

Nelson, P.H., 1999, Petrophysical Properties, Chapter PP in The oil and gas resource potential of the 1002 Area, Arctic National Wildlife Refuge, Alaska, by ANWR Assessment Team, U. S. Geological Survey Open File Report 98-34.

Schuenemeyer, J.H., 1999a, Assessment results, in the oil and gas resource potential of the 1002 Area, Arctic National Wildlife Refuge, Alaska, U.S. Geological Survey Open File Report 98-34, CDROM.

Schuenemeyer, J.H., 1999b, Methodology, in the oil and gas resource potential of the 1002 Area, Arctic National Wildlife Refuge, Alaska, U.S. Geological Survey Open File Report 98-34, CDROM.

Schuenemeyer, J.H., 2003, Methodology and results from the assessment of oil and gas resources, National Petroleum Reserve, Alaska: U. S. Geological Survey Open-File Report 03-118. Available online at: http:/geopubs.wr.usgs.gov/open-file/of03-118/of03-118.pdf

Standing, M.B., 1977, Volumetric and phase behavior of oil field hydrocarbon systems: Society of Petroleum Engineers of AIME, Dallas.

Stewart, G.W. 1973, Introduction to Matrix Computations, G. W. Stewart, Academic Press, New York (Algorithm 3.9, p. 142.)

Verma, M.K., and Bird, K.J., 2005, Role of reservoir engineering in the assessment of undiscovered oil and gas resources - in the National Petroleum Reserve, Alaska (NPRA): American Association of Petroleum Geologists Bulletin, v. 89, no. 8, p. 1091-1111. 


\section{Appendix A. Brief description of Splus functions used in the 2005 USGS Central North Slope resource assessment}

Splus code for each program is available as text files. The file name convention is to use the Splus play file name (table 3 ) as a prefix for all of the internal play specific files. We refer to the prefix generically as pfn. Also in an Splus file function argument, the equal sign $(=)$ indicates a default values, such as $p n=3$ below.

\section{Input data from Excel play form spreadsheet.}

Purpose: Reads play parameters from sheet WorkS1 into Splus

Function call: Fndata_AMdata.fn(flin, pn $=3$ )

Input:

flin is name of Excel file

pn is worksheet page number

\section{Estimate distribution parameters}

Purpose: Estimates model parameters (closure, net reservoir thickness, porosity, trap fill, depth, and number of prospects) using a beta distribution.

Function call: Fnpare_AMpare.fn("pfn")

\section{Simulation}

Purpose: Simulation of play. Generates oil and gas fields and derivatives.

pfnsim_AMsim.fn(pfn, seed $=16, \mathrm{np}=10000)$

\{

\# seed is random number seed (between 0 and 1023)

$\# \mathrm{np}=$ number of simulation runs

\section{Simulation summary statistics}

Purpose: Summaries field simulation output (pfnsim).

pfnplay_AMplay.fn_(pfn)

Output file contains one line per simulation run.

Play summary statistics:

pfnpss_AMpsum.fn_(pfn, $\mathrm{np}=10000)$ 


\section{Size-frequency tables:}

pfnfreqO_AMfreq.fn(pfn, 1, np = 10000)

\# size-frequency distribution for oil

pfnfreqG_AMfreq.fn(pfn, 2, np = 10000)

\# size-frequency distribution for nonassociated gas

\section{Size-frequency histograms:}

AMsf.fn_(pfn,og)

$\#$ og $=1$ for oil, $=2$ for na gas

Programs to generate size-frequency accumulation distributions:

pfnlnO_AMacc.fn"(pfn, 1, ns = 10000)

\# Also generates oil accumulations

\# ns is number of accumulations to be generated

pfnlnG_AMacc.fn"(pfn, 2, ns = 10000)

\# Also generates na gas accumulations

\# ns is number of accumulations to be generated

Programs to generate size-frequency accumulation plots:
AMlogac.fn(pfn, og)
\# Output is in log base 2 size classes.
\# og=1 for oil, =2 for na gas

\section{Distributions of accumulation attributes within specified range:}

AMboxpd.fn(pfn, og)

\{

\#Program reads data file and produces boxplots near specified value of

\# volume

\# $\mathrm{og}=1$ for oil, $=2$ for na gas 
Checks play correlation matrix, makes adjustment as necessary and produces sample numbers:

AMrand_AMcorr.fn(AMcorrM, AMplayr, seed, cns = 10000)

\# AMrand is sample numbers

\# AMCorrA is alternative output file and is the adjusted correlation matrix

\# AMcorrM is user specified correlation matrix

\# AMplayr contains Splus play names and play risks

\# seed is random number seed; seed between 0 and 1023

\# cns is number of simulations

\section{Aggregates play results}

AMag_AMagg.fn(AMrand, AMplayr)

Output file contains aggregate results for oil, na gas, derivatives, and corresponding sample numbers.

\section{Aggregate summary statistics:}

AMss_AMsummary.fn(AMag)

Summarizes aggregate file; means, quantiles

Output is summary file 


\section{Appendix B. Instructions to assessors concerning the protocol for the 2005 USGS Central North Slope Assessment}

By John H. Schuenemeyer

Date: 26-Jul-04

\section{INTRODUCTION}

The purpose of this protocol is to provide structure and encourage documentation for the 2005 USGS assessment of the Central North Slope in northern Alaska. It describes the information required on the play assessment form, and suggests input order. Issues of geologic analogs, uncertainty, dependencies, and risking are discussed. The form used in this assessment is a revision of the USGS ANWR-1002 (Schuenemeyer, 1999) and NPRA assessment forms (Schuenemeyer, 2003).

The assessment form is used to specify information needed to estimate recoverable oil, nonassociated gas and derivatives for a play. Statistical distributions for oil and nonassociated gas accumulation attributes, trap depth, and number of prospects are specified. Point estimates for recoverable oil and gas, engineering data, and play and prospect risking also need to be supplied. Pairwise dependencies between accumulation attributes may be specified. In addition, pairwise dependencies between plays need to be specified.

Revisions in the play assessment form include new, lower minimum accumulation sizes, specifying the number of oil and gas prospects separately, enhanced graphics, and additional feedback to assessors.

\section{GEOLOGIC MODEL}

Prior to completing a play assessment form, it is necessary to have a well documented geologic model. The model may be based upon geologic observation, analog, or a combination of the two.

Analogs can be used for at least two non-mutually exclusive purposes. The first is to provide guidance in the construction of the geologic model. This should be done first. The second is to supply missing data. Analogs are used to ascertain risking, and supply distributions including accumulation attributes, trap depth and number of prospects. For the remainder of this discussion, we address the issue of using an analog to supply data to a model. There are at least four somewhat separate issues that need to be considered.

1. Comparing an analog to a frontier area. The Ulmishek Harrison (1984) paper provides insight into a structured comparison mechanism using ranking of attributes in the analog with those in the frontier area.

2. Often multiple analogs are proposed for a given data element or set of elements and a question arises about how to choose among these. In addition to the obvious need for discussion, perhaps a comparison of the ranking of elements as 
described above would be useful. We strongly advocate that initially assessors consider multiple analogs. (See Attanasi and Freeman, 2004.)

3. Assessors need to scale the analog appropriately.

In many plays, data is derived from a combination of hard data, such as seismic observations, and analog. There is need to carefully document the components of the input that have been derived from analogs and those that are derived from seismic or other direct methods of observation. See for example, Houseknecht and Schenk (1999). I suggest that the following information be obtained and recorded for each data element (or perhaps class of data elements) used in the assessment.

1. Information source. Analog or direct observation.

2. Level of confidence in information. If analog, how closely does the analog match the frontier (forecast) area?

3. Multiple analogs. How are analogs compared? Ranked? How are disagreements, if any, resolved?

4. Scale. How is the analog scaled?

Attanasi and Freeman (2004) have prepared analogs using 36 U.S. oil plays and 25 U.S. gas plays, each containing at least 50 accumulations of MAS or greater. The information on each play includes the numbers of accumulations and volume of resource by primary and secondary lithology and trap type. The histogram of the number of accumulations (discovered and undiscovered) versus size class and the complement of the corresponding cumulative size distribution are given. Assessors should review this information for consideration as analogs to plays in northern Alaska.

\section{PLAY FORM SHEETS}

A brief description is provided for each of the 14 worksheets in the Excel assessment form.

1. Contents. Lists worksheets comprising the assessment form.

2. Shapes. Lists and describes the shapes of distributions to be used in completing assessment form.

3. WorkS1. Used to transfer information to and from Splus for computation. No user input is permitted.

4. Oil. Input specification for distributions of accumulation attributes and trap depth. Also, oil accumulation characteristics are specified as point estimates.

5. OilGraph. It has two sets of graphs. One set is the empirical density function and a fit to a beta distribution for oil accumulation attributes, trap depth, and number of oil prospects distribution. (The role of the beta distribution will be explained 
shortly.) The second set is the corresponding complementary cumulative distribution functions.

6. OilDet. Assessor may specify pairwise dependencies between the four hydrocarbon attribute distributions. Oil fvf versus trap depth is graphed.

7. Gas. Input specification for distributions of accumulation attributes and trap depth. Also, gas accumulation characteristics are specified as point estimates.

8. GasGraph. It has two sets of graphs. One set is the empirical density function and a fit to a beta distribution for oil accumulation attributes, trap depth, and number of oil prospects distribution. The second set is the corresponding complementary cumulative distribution functions.

9. GasDet. Assessor may specify pairwise dependencies between the four hydrocarbon attribute distributions. Gas fvf versus trap depth is graphed.

10. Prosp. Specify the number of oil and the number of gas prospect distributions greater than or equal to the minimum accumulation size.

11. PPR. Specify the oil and gas, play and prospect risks. Specify land, oil, and gas allocation into percents state and native lands, onshore, and offshore.

12. DistnGr. These illustrate the distributions of the fitted beta distributions and statistics for the accumulation attributes, trap depth, and number of prospects for oil and gas. (Graphs are generated by Splus - no input is required.)

13. OilAccum. This shows oil accumulation distribution and distribution of accumulation attributes near the MAS. (Graphs are generated by Splus - no input is required.)

14. GasAccum. This shows nonassociated accumulation distribution and distribution of accumulation attributes near the MAS. (Graphs are generated by Splus - no input is required.)

\section{UNCERTAINTY AND VARIABILITY}

In the assessment form, assessors are asked to specify distributions for accumulation attributes, trap depth, and number of prospects for oil and gas. The spread and shape of these distributions reflect two types of uncertainty. One is aleatory or irreducible uncertainty. We call this natural variability or just variability. It is uncertainty that cannot be reduced by increased knowledge. An example is the variability that exists in area of closure among prospects in a play. The second is epistemic uncertainty. This is uncertainty that results from lack of knowledge. This uncertainty may include measurement error, measurement bias, disagreement among assessors, and general lack of knowledge about a physical situation. Epistemic uncertain can be reduced by additional sampling, improved measurement techniques, field study, and the like.

Input distributions specified for this assessment reflect variability and uncertainty. For example, consider area of closure. As previously mentioned, there is natural variability within a play. There is also uncertainty due to measurement error (the geophysics and/or interpretation) and sampling (density of seismic lines). 
Usually it is not possible to separate variability and uncertainty. If we possessed perfect knowledge, a distribution spread would comprise only variability. Uncertainty is specified by widening the domain of the data and/or flattening the distribution.

Display 1 is an example of the specification of area of closure that illustrates variability at three levels of uncertainty. Lack of knowledge is reflected by increasing the probability in the right tail of the distributions.

\begin{tabular}{|c|c|c|c|c|c|c|}
\hline \multicolumn{7}{|c|}{ Display 1. Knowledge level - lengthen right tail } \\
\hline \multirow[b]{2}{*}{ ATTRIBUTE } & & Knowledge & & \multicolumn{2}{|c|}{$\begin{array}{c}\text { Probability (Attribute } \\
>=\text { Value) }=\end{array}$} & \\
\hline & Shape & Level $1-3^{5}$ & LTP & 0.5 & 0.05 & Max \\
\hline AREA OF CLOSURE & 1 & 1 & 2 & 4 & 8 & 10 \\
\hline AREA OF CLOSURE & 1 & 2 & 2 & 4 & 8 & 12 \\
\hline AREA OF CLOSURE & 1 & 3 & 2 & 4 & 8 & 14 \\
\hline
\end{tabular}

Lack of knowledge also may be reflected by increasing the value of the F05 (0.05) value.

When the attribute to be specified has a bounded domain, for example trap fill, which is specified as a percent between 0 and 100, an increasing degree of uncertainty is specified by making the probability more uniform. Display 2 shows how lack of knowledge may be reflected in the situation where the domain is bounded.

\section{Display 2. Trap fill, high and low knowledge levels}

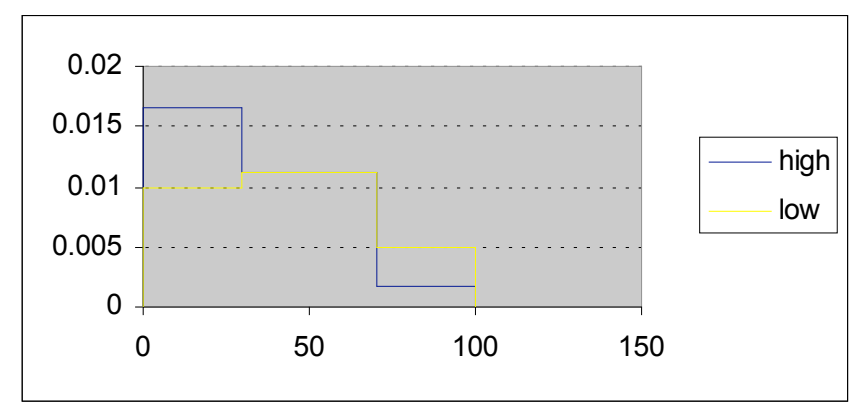

In the case of trap fill, the lower level of knowledge is reflected in the probability being more spread out, i.e., more uniform.

\section{ACCUMULATION ATTRIBUTES}

Accumulation attributes for oil and gas are input as distributions. Oil distributions are to be specified on the Oil worksheet and nonassociated gas distributions on the Gas worksheet. The suggested order for specifying the probability densities via fractiles is 1) the expected shape of the distribution, 2) knowledge level, and 3) the points on the distribution. 


\section{Shapes of Conjectured Distributions}

Shapes are described on the Shape worksheet. They may vary from highly right (positively) skewed, including J-shaped and lognormal/gamma looking, to normal, uniform, and left (negatively) skewed. The shape characterizes the overall behavior of the data in a probabilistic sense. Two shapes ( 7 and 8 on the Shape worksheet), which are mixtures of discrete and continuous, are reserved for trap fill.

The beta distribution serves as a smoothing function. Samples for the simulation used to generate an accumulation distribution and for trap depth and number of prospects will be obtained from the fitted beta distributions. Details are provided in the methodology write up.

\section{Knowledge Level}

The knowledge level is an ordinal variable with 1 being a high level of knowledge, 2 a medium level, and 3, a low level. The reason for specifying knowledge level subsequent to the shape is that the distributions (to be specified next) need to reflect the uncertainty (as characterized by lack of knowledge) and variability. An additional reason for specifying knowledge level is to provide a basis for improving future assessments. A way to reduce uncertainty is to put additional effort into those attributes that reflect a lack of knowledge. A summary of knowledge judgments from accumulation attribute distributions in the NPRA assessment (Schuenemeyer, 2003) is presented in the Appendix to this protocol.

\section{Specify fractiles for in-place distributions}

My recommendation for the order of specification is as follows:

i. Maximum value - for area of closure an upper bound may be determined by available space. For porosity and trap fill the upper bound is $100 \%$ but of course the maximum may be specified at a lower value if appropriate.

ii. Lower Truncation Point (LTP). The LTP is specified as the minimum size of geologic interest. One purpose of the LTP is to eliminate the generation of a prospect greater than the specified minimum accumulation size (MAS) that was comprised individual attributes (thickness, closure, porosity, and fill), which were too small to generate a prospect. (The MAS will be discussed soon.) For example, without the LTP's, a random sample from the hydrocarbon attribute distribution could retrieve a very small porosity ( say $<2 \%$ ), coupled with a large area of closure (say $>50,000$ acres) that would result in a "prospect" of at least MAS but from a geological viewpoint would not be viable. A second purpose is to eliminate the generation of very small accumulations, which are not judged to be of economic or technological significance in the foreseeable future. The LTP is a geologically based constraint. Only an accumulation attribute at least as large as the LTP will be generated by Monte Carlo sampling and used to generate a distribution of accumulation (prospect) sizes. These distributions are anchored at zero. 
iii. Median value. (The median is the value $\tilde{x}$ such that $\operatorname{Prob}(\mathrm{X} \geq \tilde{x})=0.5$; it is also known as F50, the $50^{\text {th }}$ fractile.) The median value is with respect to the LTP.

Thus, 0.5 probability is between LTP and the median and 0.5 is between the median and the Max value.

iv. Prob. Attribute $\geq 0.05$ - This is a value $\mathrm{x}_{0.05}$ such that $\mathrm{P}\left(\mathrm{X} \geq \mathrm{x}_{0.05}\right)=0.05$. It is also known as F05, the $5^{\text {th }}$ fractile. The F05 value is with respect to the LTP.

Should hard data (direct measurements) be available on any attribute, provision will be made to specify as many fractiles as are needed to characterize the distribution.

\section{Porosity * Water Saturation Penalty}

A porosity times water saturation penalty must be entered for oil and nonassociated gas (on the Oil and Gas worksheets respectively) in the POR*Sw box. This number can range between 0 and 6 . The Hydrocarbon Pore Volume = Porosity POR*Sw.

\section{GRAPHICAL FEEDBACK}

\section{Accumulation attributes, trap depth, and number of prospects}

Once the above information is entered in the Oil and Gas spreadsheets of the Alaskan Assessment Form, a graphical representation (Displays 3 is an example) will be given showing the empirical (user specified) density function, the LTP, and beta density function fit to the empirical density function. In addition, a display (Display 4) shows the complementary cumulative distribution function (ccdf). The ccdf is a plot of $x$ versus $\operatorname{Prob}(\mathrm{X} \geq \mathrm{x})$; in Display 4, $\mathrm{x}$ is area of closure. These graphs are displayed on the assessment form in spreadsheets OilGraph and GasGraph. Assessors will have the opportunity to change input distribution specifications and changes will be reflected on the graphs.

\section{Display 3. Example density plot - area of closure for oil.}

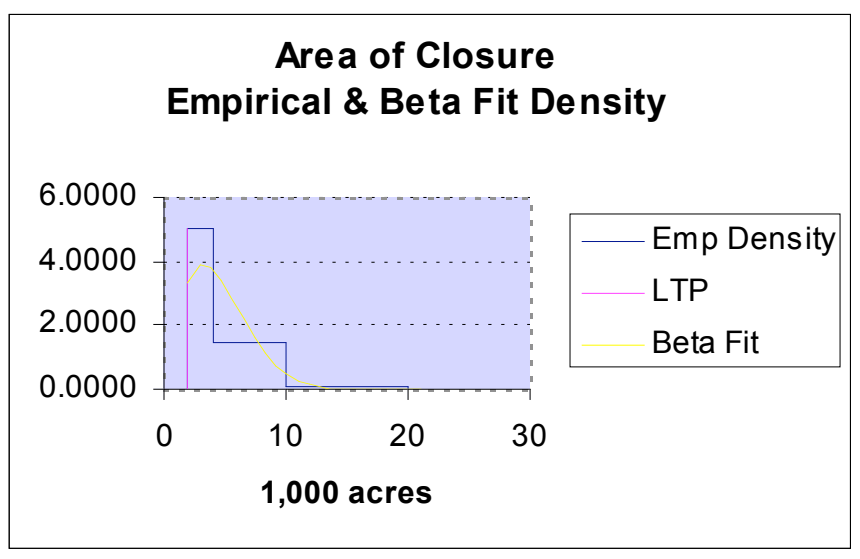




\section{Display 4. Example complementary cumulative distribution plot - area of closure for oil.}

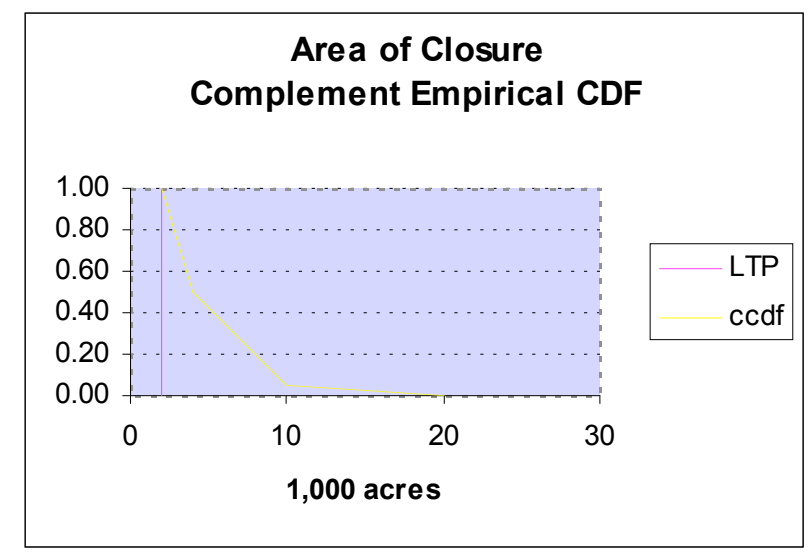

Complete graphical output is given in Excel spreadsheets OilGraph and GasGraph. To obtain a beta fit to new data requires the execution of Excel Solver (a nonlinear fitting algorithm). Solver can be found under the Tool menu. As an example, for oil Net Reservoir Thickness density graphs (spreadsheet OilGraph), the Solver equation is in cell $\mathrm{H} 7$, and the two parameters of the beta distribution are in cells $\mathrm{J} 7$ and K7. Solver may be run by clicking on Solver, setting the target cell to $\mathrm{H} 7$ and the by changing cells to $\mathrm{J} 7: \mathrm{K} 7$. Reasonable starting values are required for any nonlinear estimation algorithm to converge properly.

Note, that there will be instances when the specified shape is inconsistent with the specified fractiles. For example, an assessor may specify a complementary j-shape (shape 6) but his/her fractiles imply a normal looking distribution (shape 4). It is the assessor's responsibility to check the graph as provided via feedback to insure consistency with specified fractiles and make changes as needed.

\section{fvf graphs}

Oil fvf versus depth is graphed in the OilDet spreadsheet. The gas fvf versus depth is graphed in the GasDet spreadsheet.

\section{Accumulation size and attributes}

Technically recoverable accumulations of oil and nonassociated gas are given in spreadsheets OilAccum and GasAccum respectively. These are based upon Monte Carlo simulations from the beta distributions fit to accumulation attribute fractiles at or above the LTP's, the beta fitted trap depth distribution, fvf models, and recoverability factors. No MAS has been applied to these distributions.

In addition, distributions (in the form of boxplots) for net reservoir thickness, area of closure, porosity, and trap fill are shown for accumulation sizes near the MAS for oil 
and gas. For oil these are boxplots are generated at the F05 ( $95^{\text {th }}$ percentile) of the depth distribution. Given that the oil fvf is a shrinkage factor which increases with depth, accumulations at shallower depths will be larger. For gas the boxplots are generated at the F95 ( $5^{\text {th }}$ percentile $)$ of the depth distribution. Given that the gas fvf is an expansion factor which increases with depth, accumulations at deeper depths will be larger. Thus these graphs provide insight into ranges of accumulation attributes that generate MAS or larger.

The graphs on these spreadsheets (OilAccum and GasAccum) are generated by Splus. I am investigating the possibility of have these generated directly in Excel.

\section{SPECIFICATION OF ACCUMULATION ATTRIBUTE DEPENDENCIES}

The degree of linear dependency, if any, between accumulation attributes is specified by Pearson correlations on sheets OilDet (Oil Accumulation Attribute Dependencies) and GasDet (Gas Accumulation Attribute Dependencies). Values between -1 and +1 are permitted. A check will be made to ensure that the pairwise correlations are mutually consistent. Sampling to generate accumulations will reflect the specified dependencies. High (near one) positive dependencies will result in a greater spread (range) of accumulation values than smaller positive, zero, or negative correlations. Although nonlinear dependencies may exist, these will be approximated by the specification of linear dependencies.

\section{TRAP DEPTH}

Specification of the trap depth distributions for oil (Oil worksheet) and gas (Gas worksheet) is similar to that of the accumulation attribute distributions except that in place of a LTP, a minimum (Min), which typically will be greater than zero, is specified. The resultant distribution will then be shifted by the minimum value specified. Depth distributions are with respect to the surface. A surface to sea level correction is required and will be added to equalize depth distributions among plays.

\section{NUMBER OF OIL AND GAS PROSPECTS}

The oil and gas prospect distributions are to be specified separately by considering oil and gas as separate subplays whenever possible. These separate distributions are to be entered on the Prosp worksheet. The order of specification of distribution is the same as that for the trap depths.

For oil, the numbers of prospects to be specified are for technically recoverable sizes of at least 5 million barrels recoverable. For nonassociated gas, the numbers of prospects to be specified are those at least 100 bcf recoverable. These numbers, $5 \mathrm{~mm}$ bbl technically recoverable for oil and $100 \mathrm{bcf}$ technically recoverable for nonassociated gas, are the Minimum Accumulation Sizes (MAS's). Only prospects of at least these sizes will be generated by the simulation model and become part of the final assessment. 
A check is provided, which compares non-overlapping area of closure at the median and F05 for the specified numbers of prospects with the play area. Of course given that prospects occur at varying depths, some stacking may occur making this check conservative.

\section{OIL ACCUMULATION CHARACTERISTICS AND NONASSOCIATED GAS CHARACTERISTICS}

Three of the most critically specified point values are the oil and gas recovery factors (percent technically recoverable) and the API gravity (for oil). A critical computed variable, especially for gas, is the formation volume factor (fvf). For oil, the fvf serves to shrink the volume as it moves from in-place to the surface. For gas, it expands it. Equations and graphs of fvf's versus trap depth are given in worksheet OilDet and GasDet for oil and gas respectively. The gas-to-oil ratio (GOR) is a computed value. The equations used to compute the fvf's are those used in the NPRA assessment and were supplied by USGS petroleum engineer Mahendra Verma with input from assessment geologists. The fvf models can be made play specific.

\section{PLAY AND PROSPECT RISKING}

The complement of risk, "1-risk" is specified, namely the probability of a favorable outcome. There are two sets of risks - a play risk and a prospect risk. Both are specified on the PPR worksheet. The play and prospect risks, which are assumed to be independent, are a product of three independent factors: charge, trap/rock, and timing. Play favorability means that conditions exist for at least one accumulation of MAS or larger. It does not guarantee the presence of one or more accumulations.

If the play is partitioned into an oil and a gas subplay, separate risking structures can be specified for oil and gas. Play favorability should be specified first and prospect favorability second.

The prospect level favorability also is defined as a prospect of at least MAS. Oil and gas prospects can be risked separately, even when only one play is defined. Care must be taken to avoid double risking.

\section{PLAY AGGREGATION}

The procedures outlined in the NPRA (and ANWR-1002) methodology section will be used. Assessors will be asked to specify dependencies between each pair of plays. Specifically, assessors will be asked to assign one of three ordinal correlations (low, medium or high) to the attributes of charge, trap, and timing. A high positive correlation assigned to charge between, say plays A and B may indicate that a common mechanism charged both plays. Thus, if charge in play A was found to be high, the charge in play B would most likely be high. High, medium, and low correlations will be assigned values of $0.9,0.5$, and 0.1 respectively. A combined correlation matrix will be formed by taking the arithmetic average of the three correlation matrices. There will be 
one matrix each for charge, trap, and timing. Negative dependencies are not allowed. The combined (mean) correlation matrix will be tested for internal consistency (in statistical parlance, this means that the matrix must be positive definite). Should it be inconsistent, small biases will be introduced to make the matrix internally consistent. Results will be provided to the responsible assessors for feedback and confirmation.

When a play crosses a geopolitical boundary of interest, the percent of volume allocated to each subdivision of the play is specified. Separate allocations can be made for oil and gas. (See assessment form PPR sheet).

A sample of normal distributions with correlations of $0.1,0.5$ and 0.9 is shown in the following graph (Display 5).

Display 5. Sample scatter plots showing correlations of $0.1,0.5$, and 0.9 .
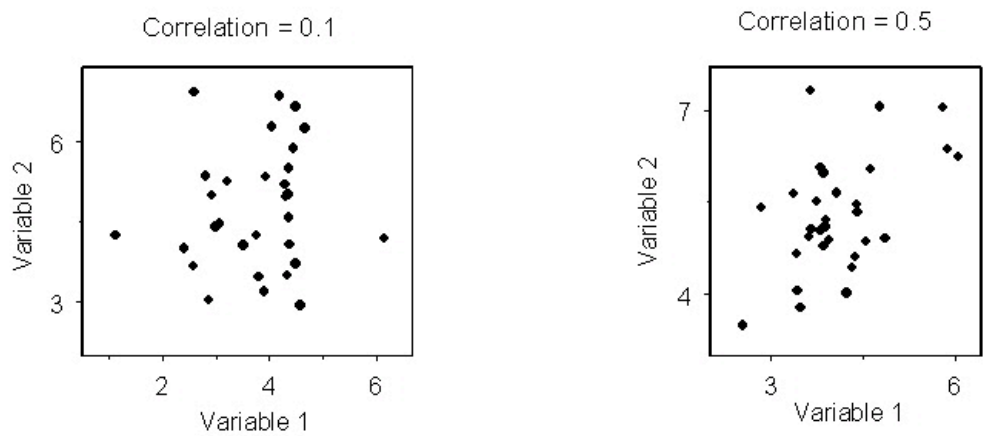

Correlation $=0.9$

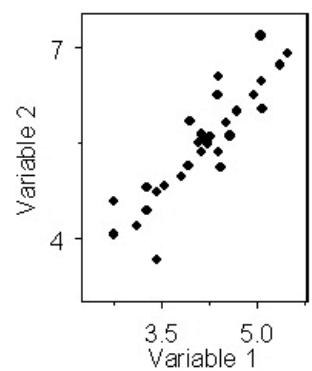

\section{SUMMARY RESULTS}

Following the final assessment meeting, I will prepare summary sheets that will include summary input: final accumulation attribute and depth distributions, number of prospects distributions, risking and other key information specified on the assessment forms; and summary output including size-frequency distributions by play. The purpose of these summary sheets will be to allow assessors and other reviews to view the entire suite of plays and thus check for overall consistency. 


\section{References}

Attanasi, E.D., and Freeman, P.A., 2004, Size-frequency analysis of petroleum accumulations in selected United States plays: Potential analogues for frontier areas: U. S. Geological Survey Open-File Report 2004-1299 (available on-line at http://pubs.usgs.gov/of/2004/1299/, 163 p.

Houseknecht, D.W. and Schenk, C.J., 1999, Chapter P1 Topset Play, in The Oil and Gas Resource Potntial of the Arctic National Wildlife Refuge 1002 Area, Alaska, by the ANWR Assessment Team. U.S. Geological Survey Open File Report 98-34, CDROM.

Schuenemeyer, J.H., 1999, Methodology, in the oil and gas resource potential of the 1002 Area, Arctic National Wildlife Refuge, Alaska, U.S. Geological Survey Open File Report 98-34, CDROM.

Schuenemeyer, J.H., 2003, Methodology and results for the assessment of oil and gas resources, National Petroleum Reserve Alaska: U.S. Geological Survey OFR 03-118, available at http://geopubs.wr.usgs.gov/open-file/of03-118/.

Ulmishek, G., and Harrison, W., 1984, Quantitative methods for assessment of petroleum resources of poorly known basins, in Masters, C.D., ed., Petroleum Resource Assessment: Ottawa, Canada, International Union of Geological Sciences, Publication No. 17, p. 80-94.

\section{Appendix to Protocol. Summary of knowledge level for NPRA accumulation attributes.}

The following information is provided to assessors to assist them in gaining insight into uncertainty among attributes in a previous USGS assessment (Schuenemeyer, 2003).

Knowledge level - hydrocarbon volume attributes - NPRA

Oil
NET RESERVOIR THICKNESS
AREA OF CLOSURE
POROSITY
TRAP FILL

\begin{tabular}{|r|r|r|r|r|r|r|r|r|r|r|r|r|r|r|r|r|r|rrr|}
\hline 110 \\
\hline 3 & 2 & 2 & 2 & 2 & 2 & 2 & 2 & 2 & 2 & 2 & 2 & 3 & 3 & 2 & 3 & 2 & 2 & 0 & 14 & 4 \\
\hline 3 & 3 & 3 & 3 & 3 & 3 & 3 & 2 & 2 & 2 & 2 & 1 & 3 & 3 & 2 & 3 & 2 & 1 & 2 & 5 & 11 \\
\hline 3 & 2 & 2 & 2 & 2 & 2 & 2 & 2 & 2 & 2 & 2 & 2 & 2 & 2 & 1 & 2 & 2 & 2 & 1 & 16 & 1 \\
\hline 3 & 2 & 2 & 2 & 2 & 2 & 2 & 2 & 2 & 2 & 2 & 3 & 3 & 3 & 2 & 3 & 3 & 3 & 0 & 11 & 7 \\
\hline
\end{tabular}

\section{Gas}

NET RESERVOIR THICKNESS

AREA OF CLOSURE POROSITY

TRAP FILL

\begin{tabular}{|r|r|r|r|r|r|r|r|r|r|r|r|r|r|r|r|r|r|rrr|}
\hline 3 & 2 & 2 & 2 & 2 & 2 & 2 & 2 & 2 & 2 & 2 & 3 & 3 & 3 & 3 & 3 & 3 & 2 & 0 & 11 & 7 \\
\hline 3 & 3 & 3 & 3 & 3 & 2 & 2 & 2 & 2 & 2 & 2 & 3 & 3 & 2 & 3 & 3 & 1 & 2 & 1 & 8 & 9 \\
\hline 3 & 2 & 2 & 2 & 2 & 2 & 2 & 2 & 2 & 2 & 2 & 2 & 3 & 3 & 2 & 3 & 3 & 2 & 0 & 13 & 5 \\
\hline 3 & 2 & 2 & 2 & 2 & 2 & 2 & 2 & 2 & 2 & 3 & 3 & 3 & 3 & 3 & 3 & 3 & 3 & 0 & 9 & 9 \\
\hline
\end{tabular}

$$
\begin{aligned}
& 1=\text { high knowledge } \\
& 2=\text { medium knowledge } \\
& 3=\text { low knowledge }
\end{aligned}
$$

There appears to be only a slight edge in stated knowledge level of oil over gas attributes. Porosity may be known at a slightly higher level of knowledge that the other attributes, although for two oil plays and one gas play the level of knowledge on area of closure was reported as high. 


\section{Appendix C. Glossary}

The following definitions were updated by J.H. Schuenemeyer and K. J. Bird, as appropriate, from those by R. Charpentier, Definitions in The Oil and Gas Resource Potential of the 1002 Area, Arctic National Wildlife Refuge, Alaska, by ANWR Assessment Team, U.S. Geological Survey Open-File Report 98-34 and used in the 2002 USGS NPRA and the 2005 USGS Central North Slope oil and gas assessments.

Accumulation Attributes: Distributions were specified for five attributes used to calculate the volumes of oil and gas accumulations in the simulation program and in economic scenarios. Because the simulation program calculates an accumulation size using one randomly sampled number from each fitted distribution, spread in the distributions reflect variability between accumulations. Even though some attributes (net reservoir thickness, porosity, and trap fill) could show variation within an individual accumulation, that level of variability is too detailed for this analysis. The distribution of porosity, for example, shows how the average porosity in an accumulation varies from accumulation to accumulation. A sampled value of porosity could be viewed as the mean value in a given accumulation. All of the accumulation attribute distributions are conditional distributions -- conditional on both the play being favorable and the prospect being favorable. The uncertainty expressed in the specification of the accumulation attribute fractiles is not intended to reflect the chance that such an attribute will be present. This is addressed by the risking. The spread in the distributions, however, may reflect geologic uncertainty and lack of knowledge.

Net Reservoir Thickness: A distribution for net reservoir thickness (in feet) in accumulations. The distribution shows how the average net reservoir thickness changes from accumulation to accumulation. It is not the same as the prospect height because it only includes net thickness of reservoir-quality rocks. It is also not the same as net pay thickness, because only some proportion of the reservoir rock contains hydrocarbons. (See trap fill.)

Area of Closure: A distribution for area of trap closure (in thousands of acres) of accumulations.

Porosity: A distribution for average porosity (in percent) in accumulations. The distribution shows how the average porosity changes from accumulation to accumulation.

Trap Fill: A distribution for trap fill (in percent) in accumulations. It is the volumetric percent of the gross reservoir volume (area of closure times net thickness) containing hydrocarbons.

Trap Depth: A distribution for trap depth (in thousands of feet sub sea level) in accumulations. The distribution shows how the average trap depth changes from accumulation to accumulation. 


\section{Risking:}

Minimum Accumulation Size (MAS) Oil: The smallest accumulation size being assessed, in this case 5 million barrels (MMBO) technically recoverable. Smaller accumulations may exist in the play but are not being assessed.

Minimum Accumulation Size Nonassociated Gas: The smallest accumulation size being assessed, in this case 100 billion cubic feet (BCF) technically recoverable. Smaller accumulations may exist in the play but are not being assessed.

Number of Prospects: A distribution showing uncertainty in the number of prospects for accumulations of the minimum accumulation size or larger. This distribution is conditional on the play being favorable.

Play Attributes: Three probabilities -- Charge (C), Trap/Rock (T), and Timing (F) -- that are used in calculating the Play Probability.

Charge (C): The probability that there has been sufficient source rock, thermal history, and migration to allow for at least one accumulation of minimum reservoir size or larger somewhere within the play.

Trap (T): The probability of the occurrence of rocks containing suitable reservoir characteristics, sealing characteristics, and trap geometry capable of containing at least one accumulation of minimum reservoir size or larger somewhere within the play.

Timing $(\mathrm{F})$ : The probability that timing was favorable for an accumulation of minimum reservoir size or larger somewhere within the play.

Play Probability: The probability that the play is favorable, i.e., that the play attributes are adequate to allow at least one accumulation of minimum reservoir size or larger. It is calculated as the product of the three play attributes -- Charge (C), Trap/Rock (T), and Timing (F) -- which are assumed to be pairwise independent. Favorability of the product of the three play attributes is necessary, but not sufficient, for the existence of an accumulation of minimum reservoir size or larger. With a small number of prospects, there is some probability that the play attributes are favorable, but just not present in any one prospect.

Play Risk: The probability that the play is unfavorable, i.e., that the play attributes are not sufficiently favorable to allow any accumulations of minimum reservoir size or larger. It is calculated as 1 minus the Play Probability.

Prospect Attributes: Three probabilities -- Charge (c), Trap/Rock (t), and Timing (f) -that are used in calculating the Prospect Probability. All of them are conditional probabilities -- conditional on the play being favorable. Probabilities are expressed relative to a randomly chosen prospect. This can also be thought of as giving the proportion of prospects for which a particular condition is favorable. These differ from 
the play attributes. For example, one may be certain that there has been sufficient source rock, thermal history, and migration to allow at least one accumulation of minimum reservoir size or larger somewhere within the play $(\mathrm{C}=1.0)$ but estimate that only $50 \%$ of the prospects have had adequate migration paths open $(\mathrm{c}=0.5)$.

Charge (c): The probability (given that the play is favorable) that a randomly chosen prospect has been charged by fluids sufficient for an accumulation of minimum reservoir size or larger.

Trap/Rock $(\mathrm{t})$ : The probability (given that the play is favorable) that a randomly chosen prospect has suitable reservoir characteristics, sealing characteristics, and trap geometry capable of containing an accumulation of minimum reservoir size or larger.

Timing (f): The probability (given that the play is favorable) that a randomly chosen prospect has timing favorable for an accumulation of minimum reservoir size or larger.

Prospect Probability: The probability that a randomly chosen prospect is favorable (given that the play is favorable), i.e., that the prospect contains an accumulation of minimum reservoir size or larger. It is calculated as the product of the three prospect attributes -- Charge (c), Trap/Rock (t), and Timing (f) -- which are assumed to be pairwise independent. Favorability of the product of the three prospect attributes is both necessary and sufficient for the existence of an accumulation of minimum reservoir size or larger in a prospect. The prospect probability can also be thought of as giving the proportion of prospects that contain an accumulation of minimum reservoir size or greater.

Prospect Risk: The probability that a randomly chosen prospect is unfavorable, i.e., that the prospect does not contain an accumulation of minimum reservoir size or larger. It is calculated as 1 minus the Prospect Probability.

Fraction of Accumulations Being Oil: That proportion of the accumulations that will be simulated as oil accumulations as opposed to nonassociated gas accumulations.

\section{Miscellaneous:}

Accumulation: Trapped hydrocarbons in contiguous pools of a particular play. In this assessment only accumulations of minimum reservoir size or larger are being assessed.

Field: One or more accumulations whose projections on the earth's surface are the same or overlap. All the pools in an accumulation are of the same play, but a field may include pools of different plays. The simulation methodology for plays estimates accumulation sizes. Fields are important principally in the economic part of the analysis.

Play: A play is a set of known or postulated oil or gas accumulations having similar geologic, geographic, and temporal properties, such as source rock, migration pathways, timing, trapping mechanism, and hydrocarbon type. 
Play Area: A geographic area that includes all the discovered accumulations (if any) and all the prospects (if any) in a particular play.

Prospect: A feature that may contain trapped hydrocarbons of at least the minimum accumulation size. 


\section{Appendix D. Algorithms}

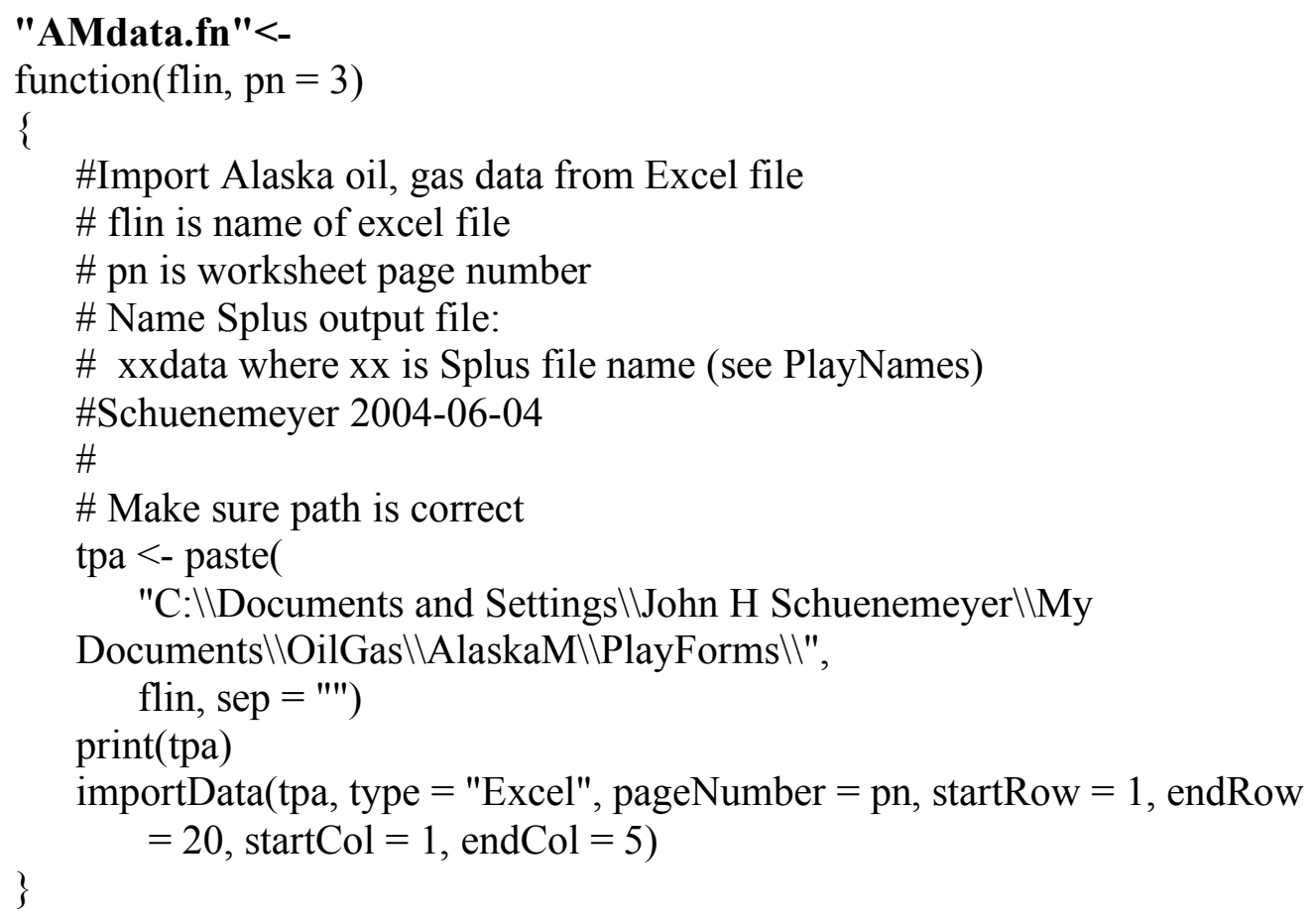




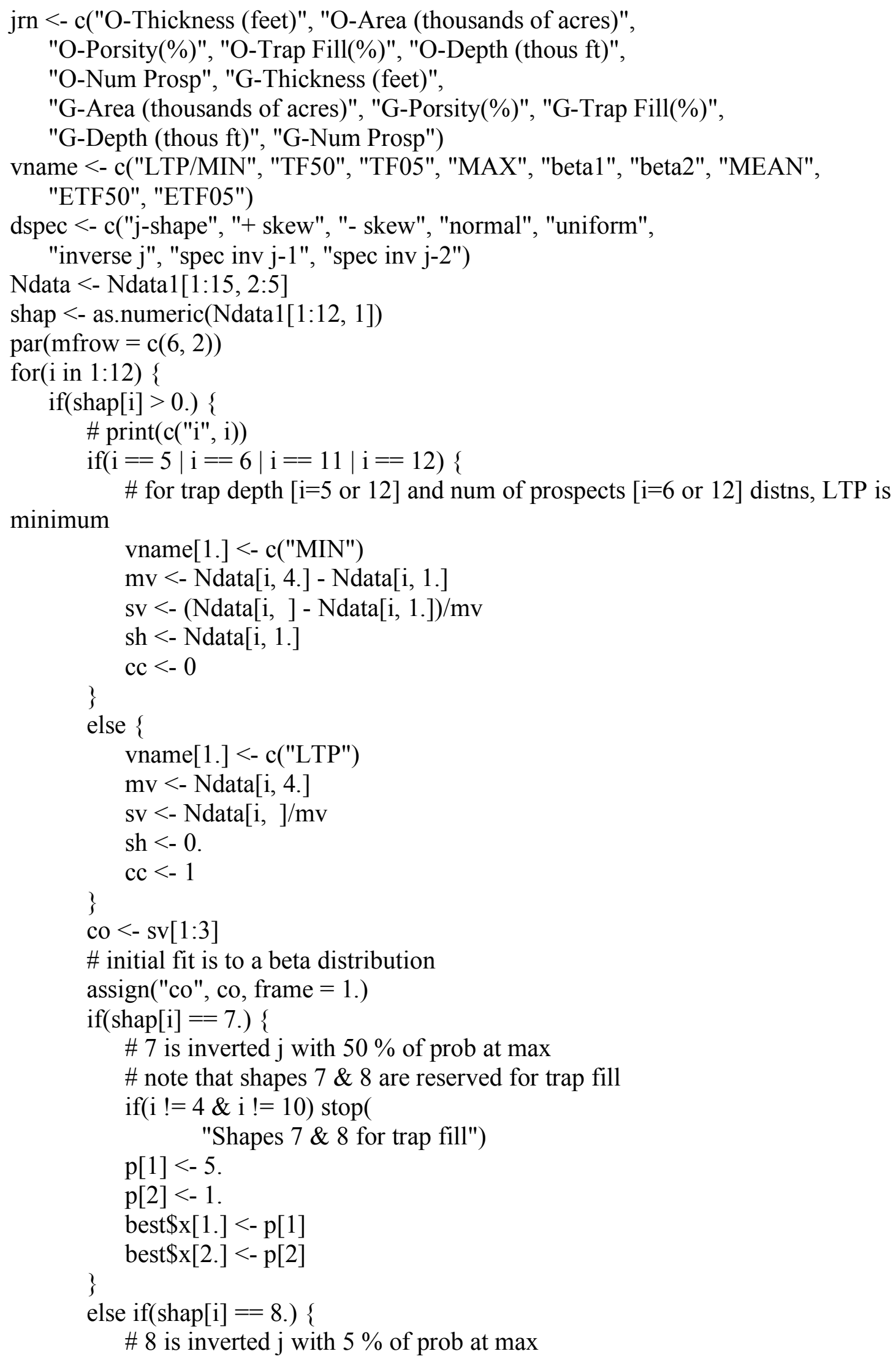




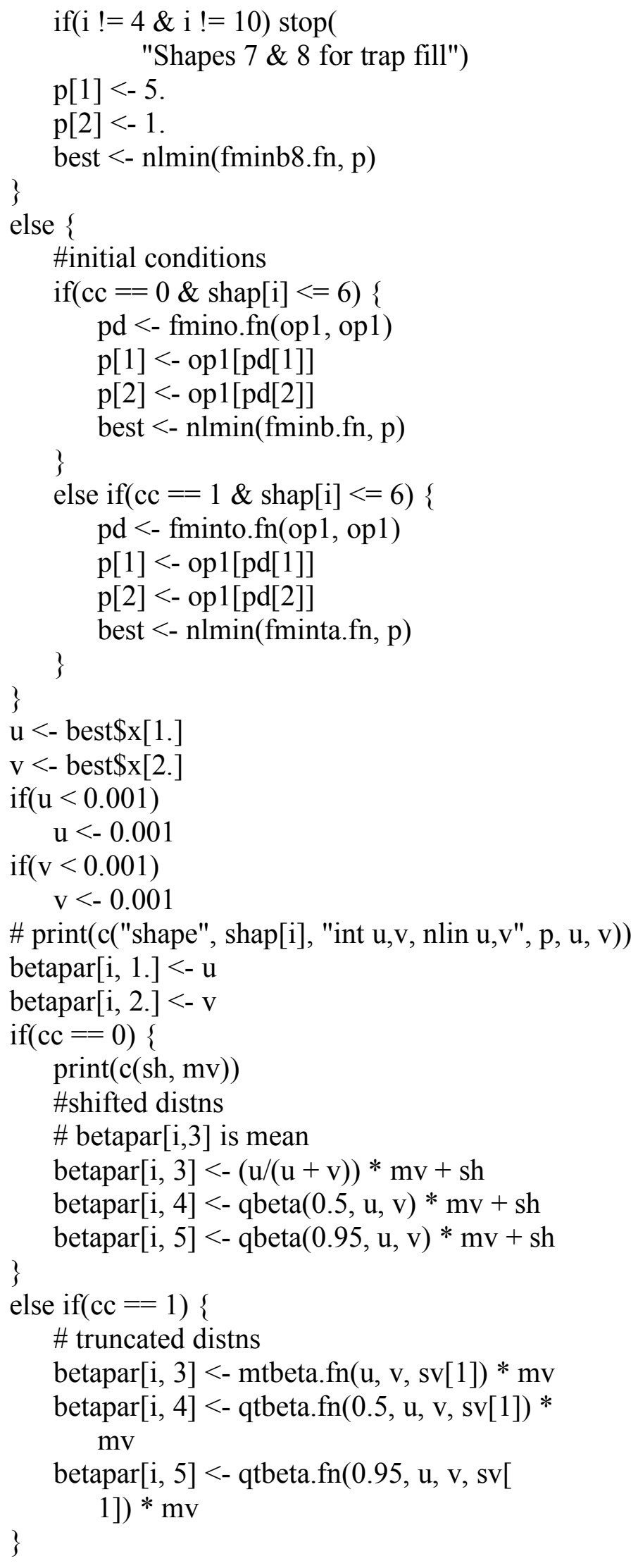




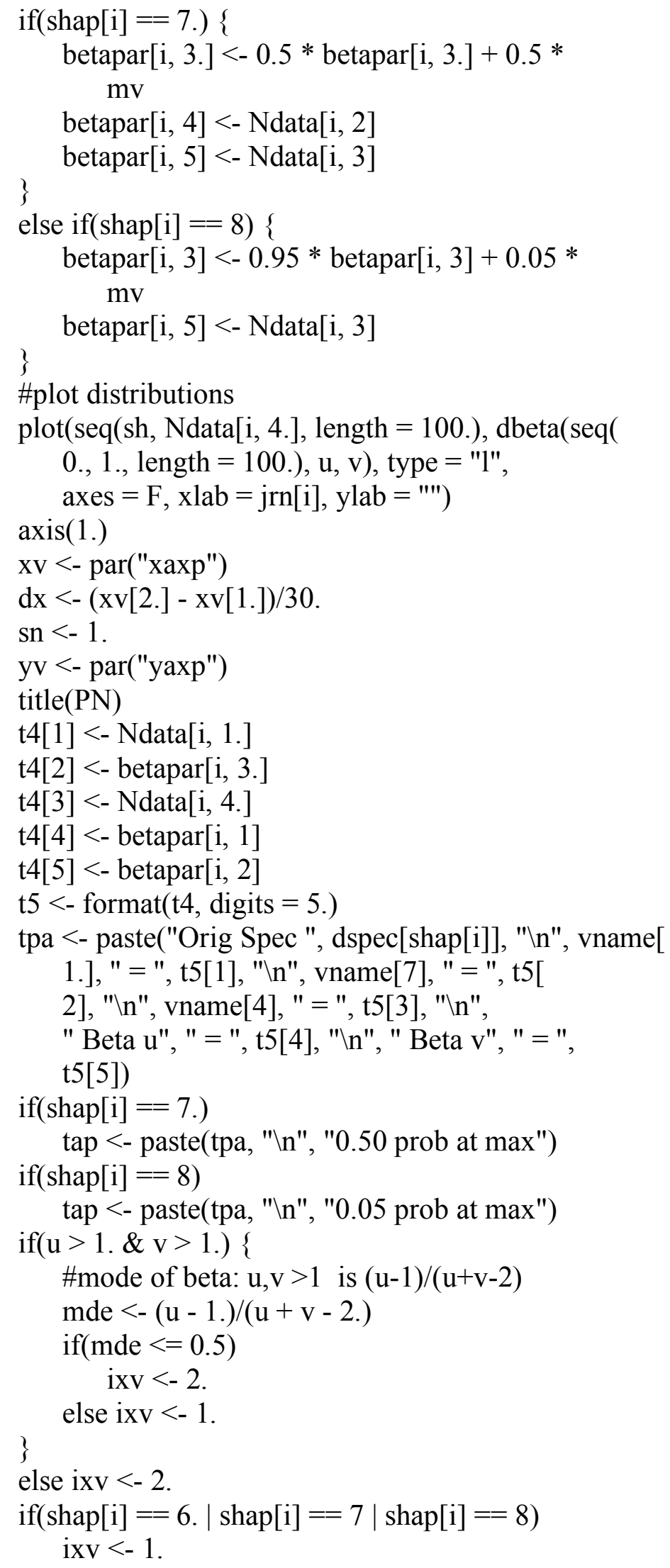




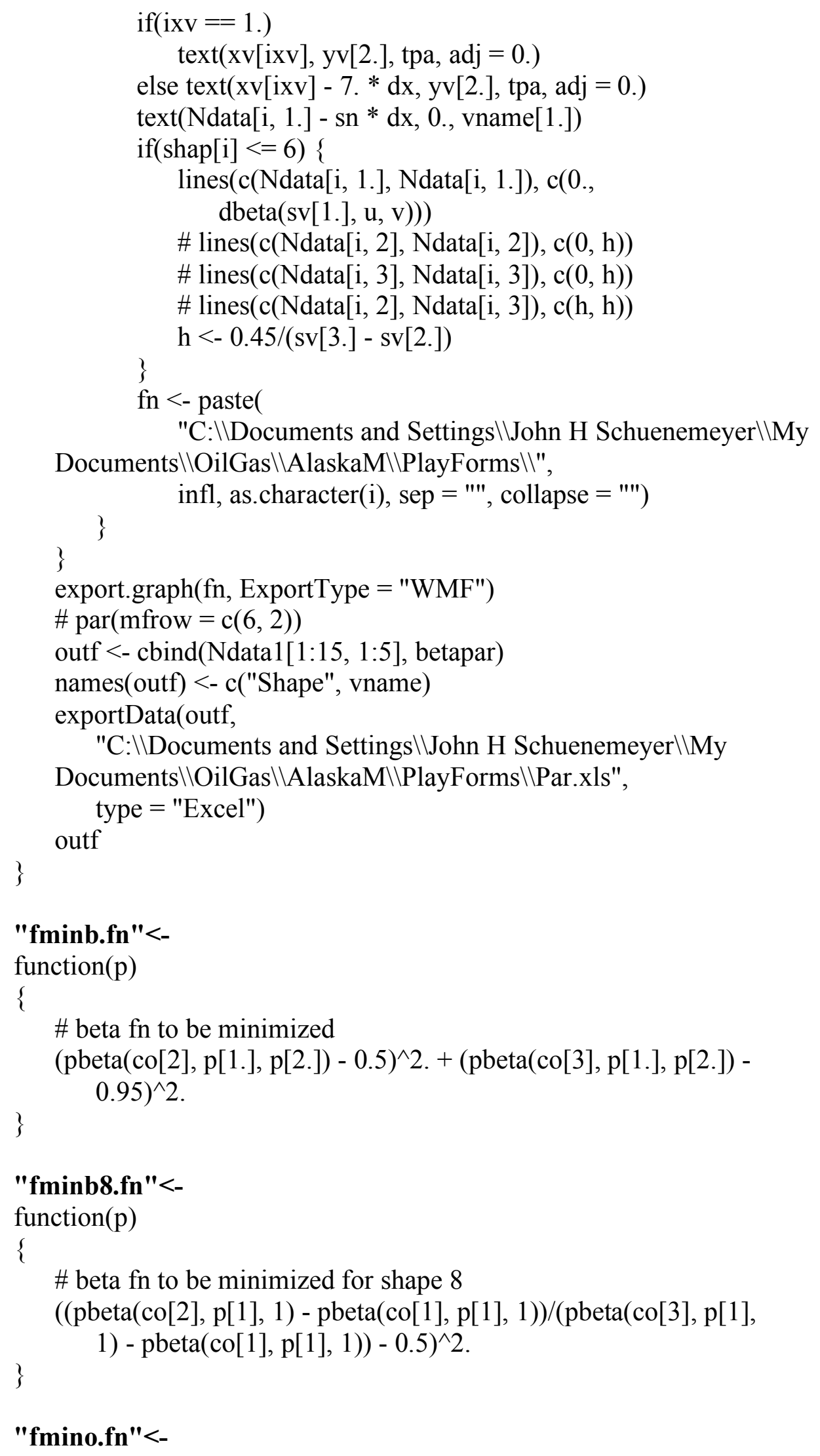




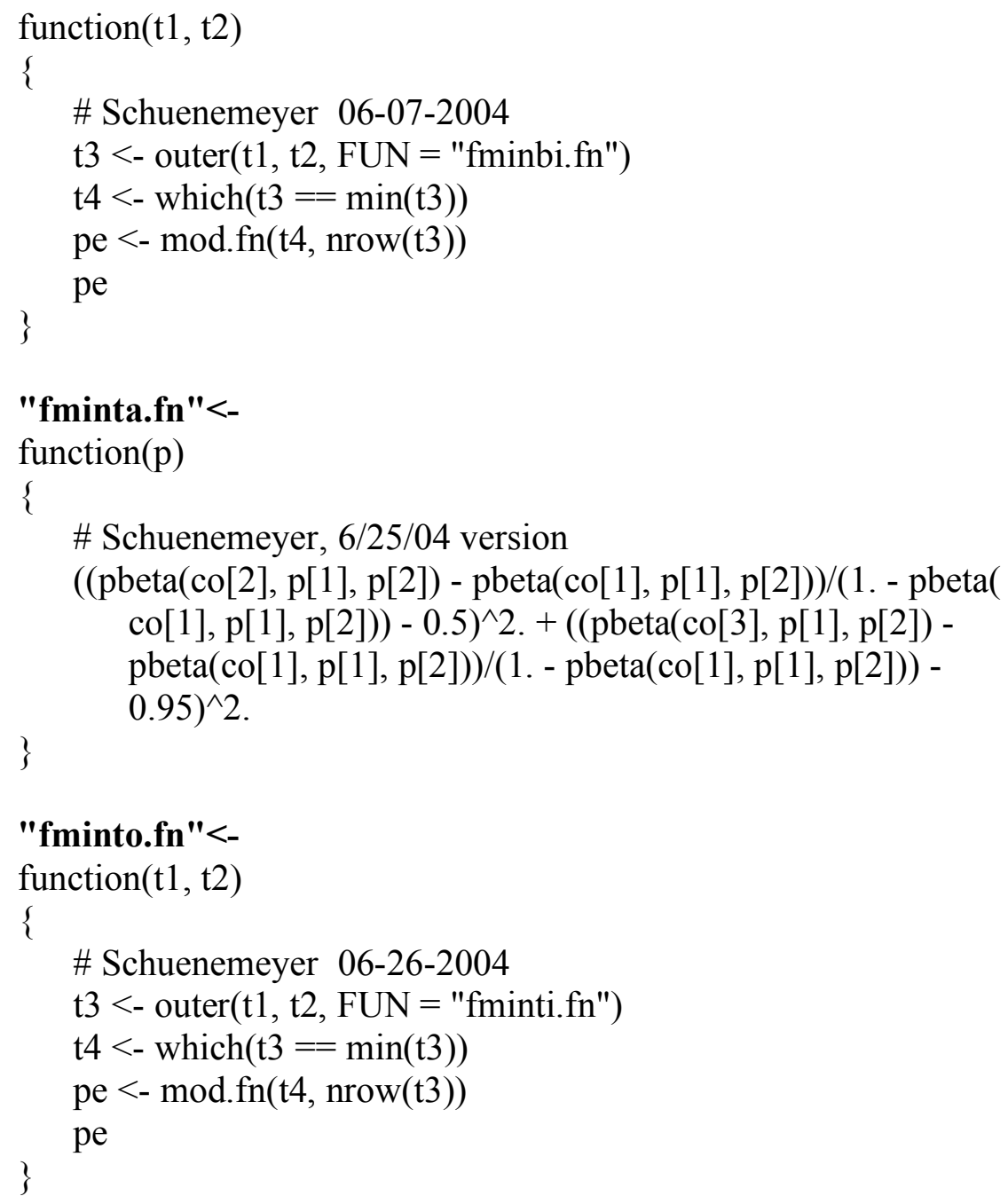




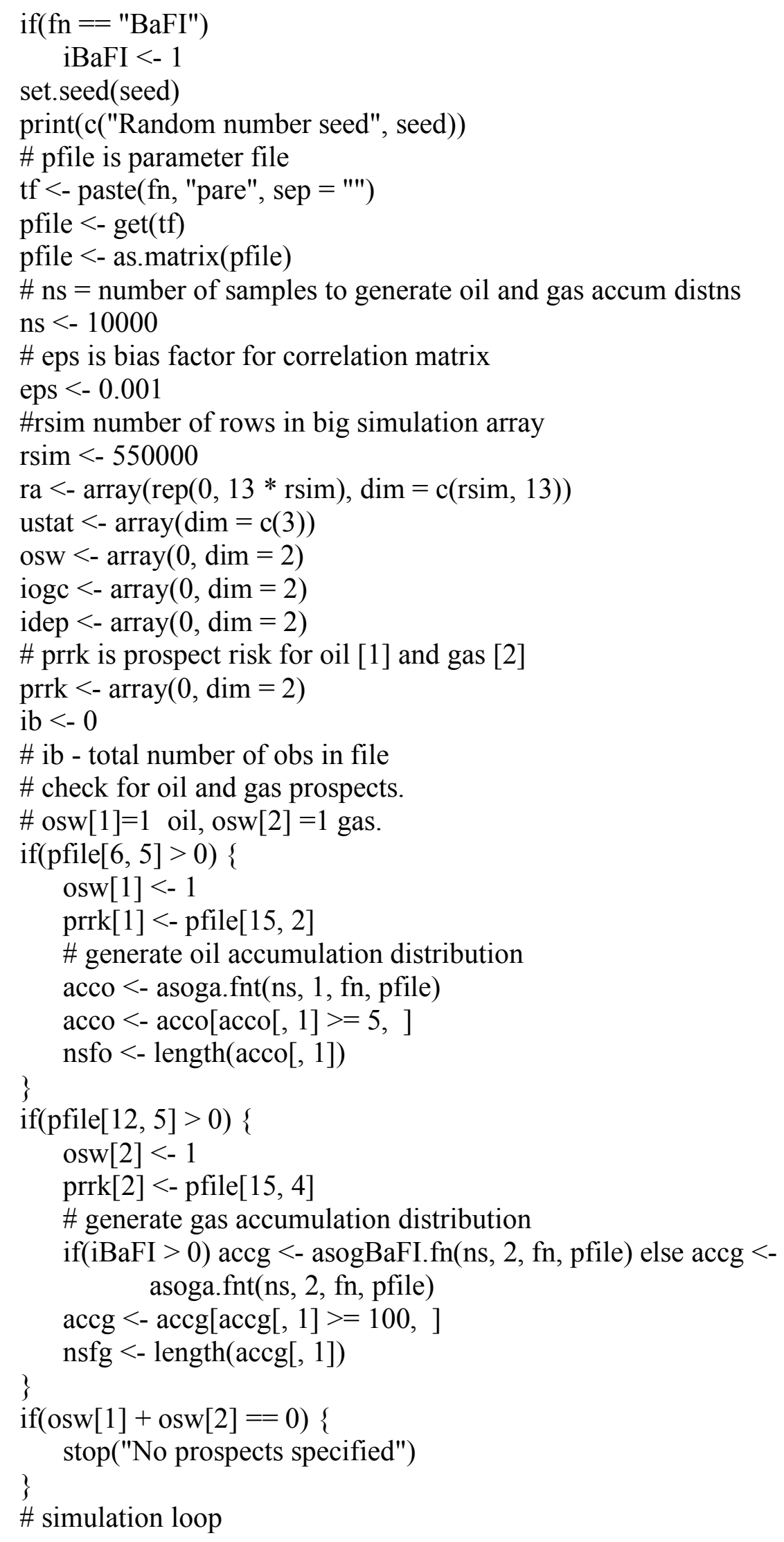




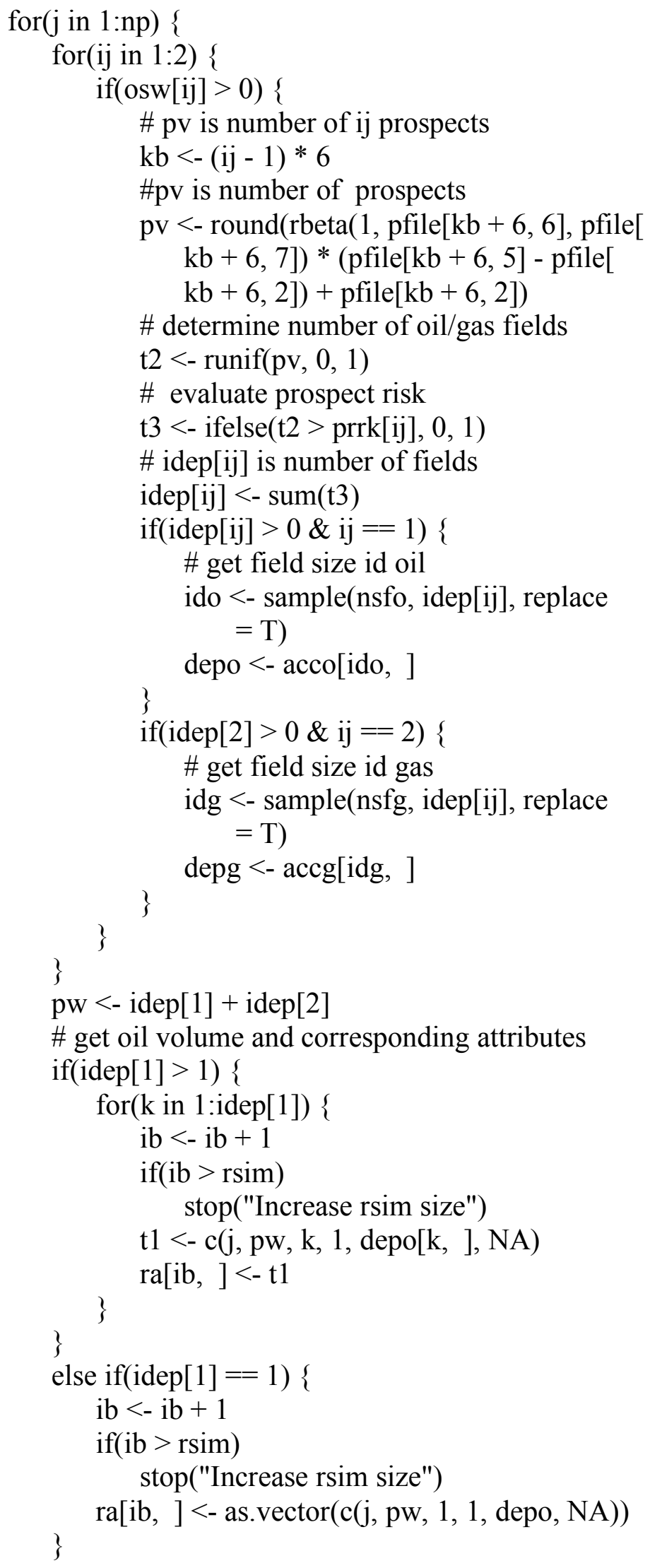




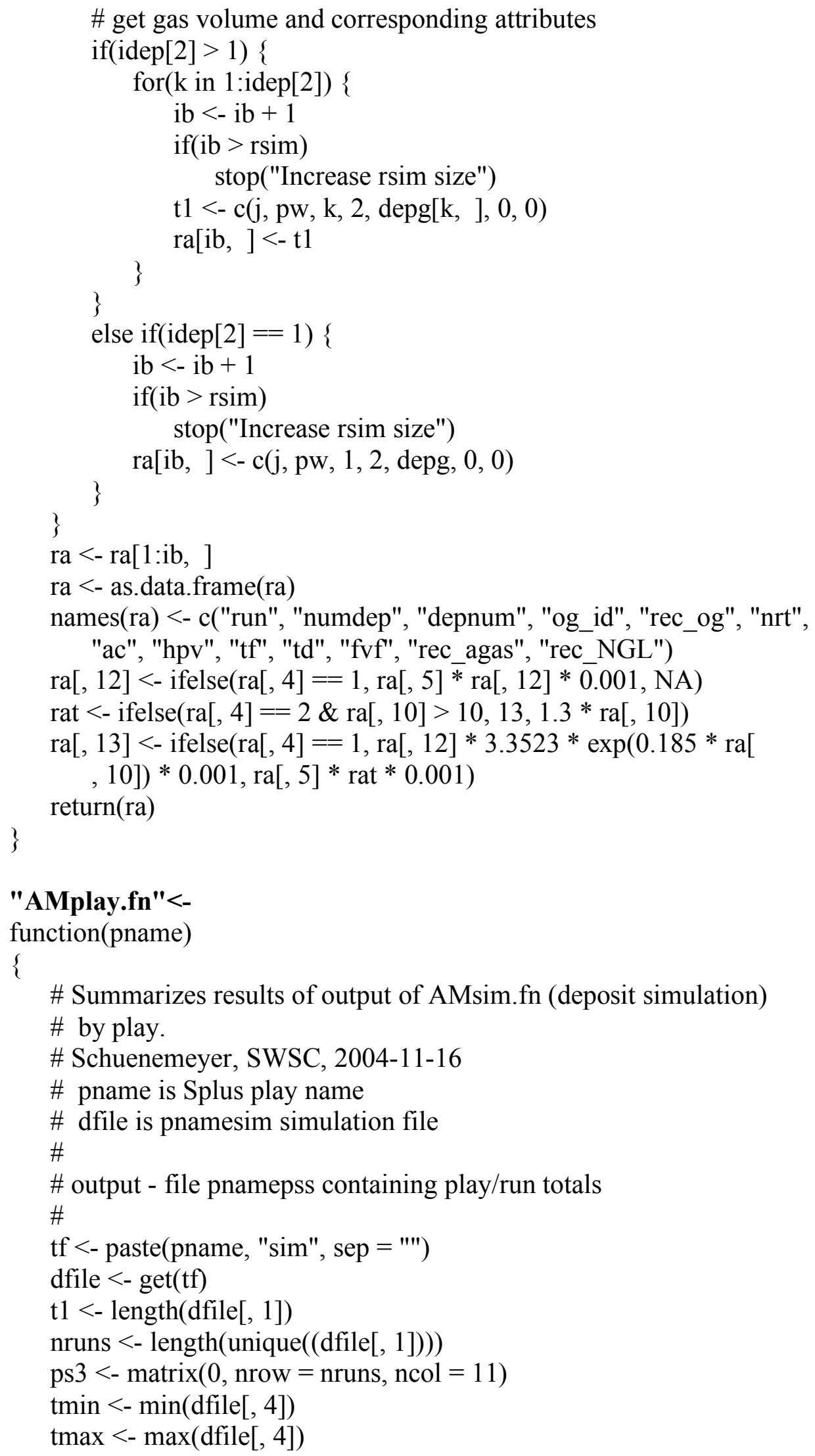




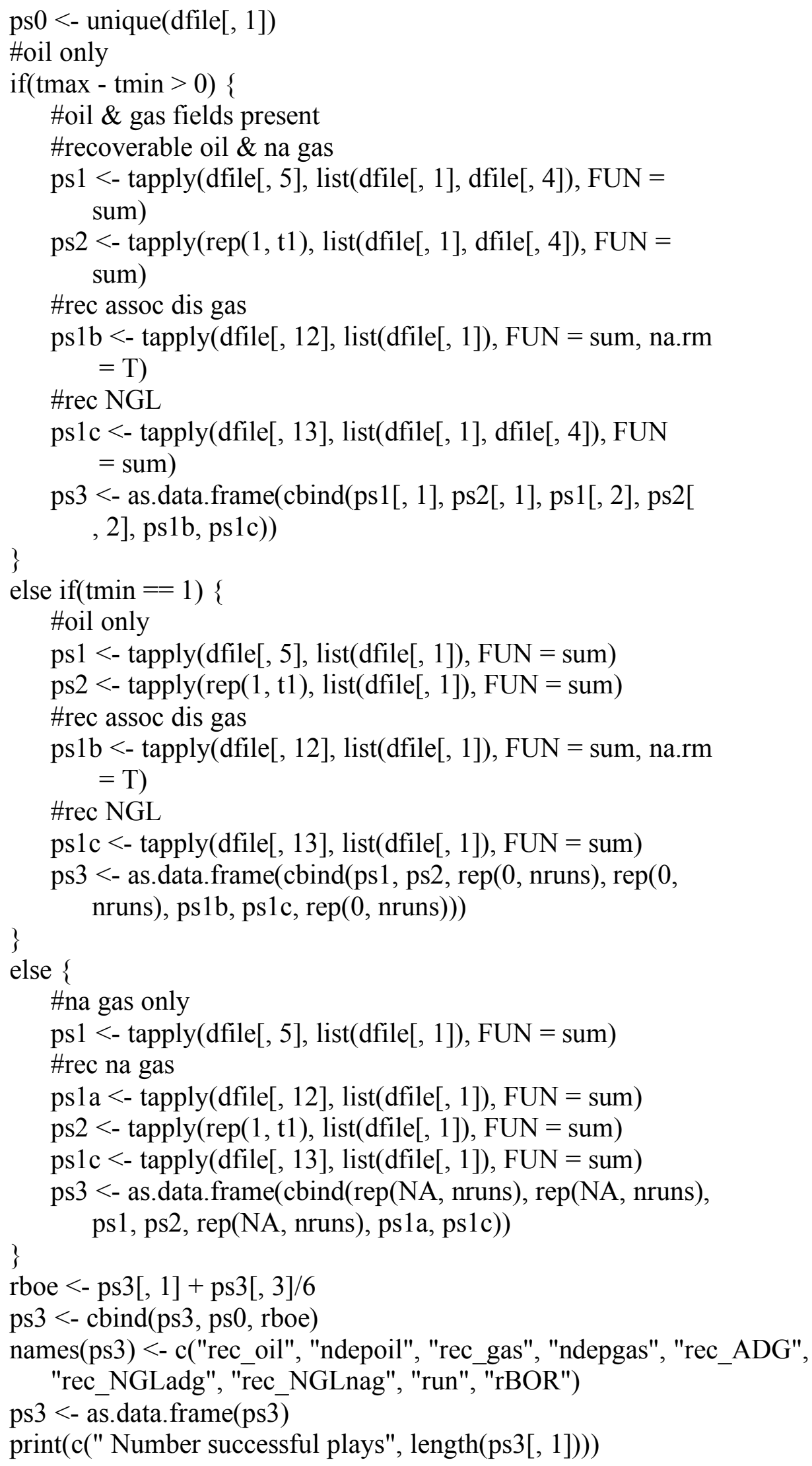




$$
\}^{p s 3}
$$

\section{"AMpsum.fn"<-}

function(pname, $\mathrm{np}=10000$ )

\{

\# summarizes play results graphically and numerically

\# output file is xxpss

\# Schuenemeyer, SWSC, 2004-10-20

\# pname is play name

\# dfile is play summary file, $x x p l a y, x x$ is Splus play name

\# dataf is basic data file, xxdata

\#

\# tplay is total number of plays=num sim runs/play favorability

\# np is number of simulation runs

tf $<-$ paste(pname, "play", sep = "')

dfile $<-$ get(tf)

tf $<$ - paste(pname, "data", sep = "")

dataf $<-$ get(tf)

playr $<-\max ($ dataf $[15,1]$, dataf[15, 3], na.rm $=\mathrm{T})$

print(c("Play risk", playr))

tplay $<$ - round(np/playr)

cv <- c("Rec Oil (mm bbl)", "Num Oil Acc", "Rec NA Gas (bcf)",

"Num Gas Acc", "Rec ADG", "Rec NGLadg", "rec_NGLnag")

ustat $1<-\operatorname{array}(\operatorname{dim}=\mathrm{c}(7,7))$

uf $<-\operatorname{array}(\operatorname{dim}=\mathrm{c}(7,8))$

\#compute recoverable oil and gas

\#compute summary stats

for(i in 1:7) \{

actog $<-$ na.omit(dfile[, i])

actp $<-$ length $($ actog $)$

if $(\operatorname{actp}>0)\{$

if(actp $<$ tplay)

actog $<-$ append(actog, rep $(0$, tplay - actp +

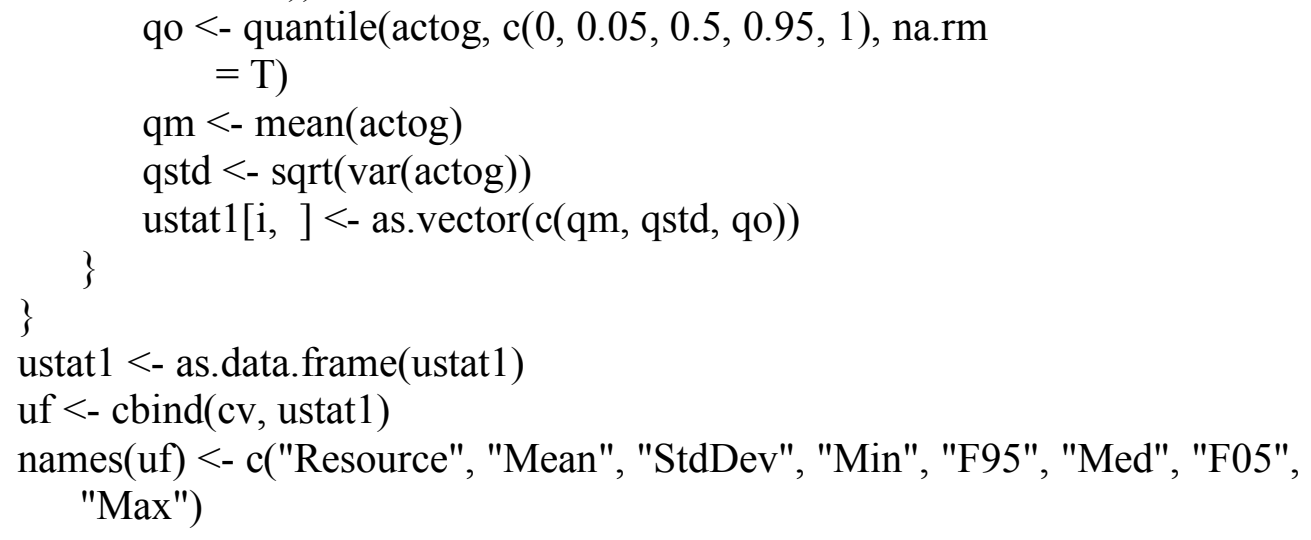




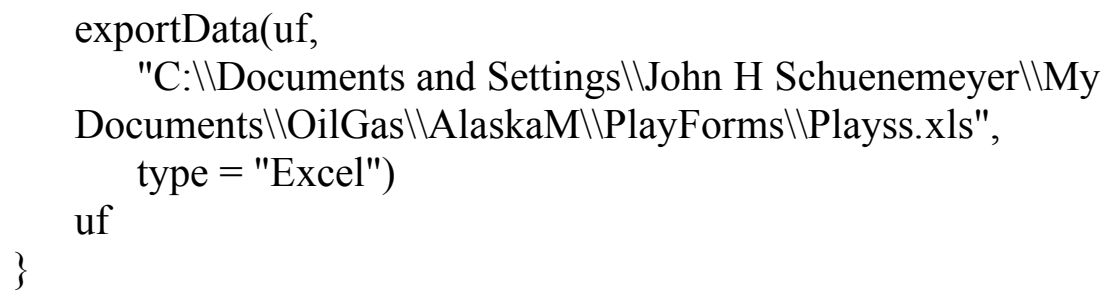




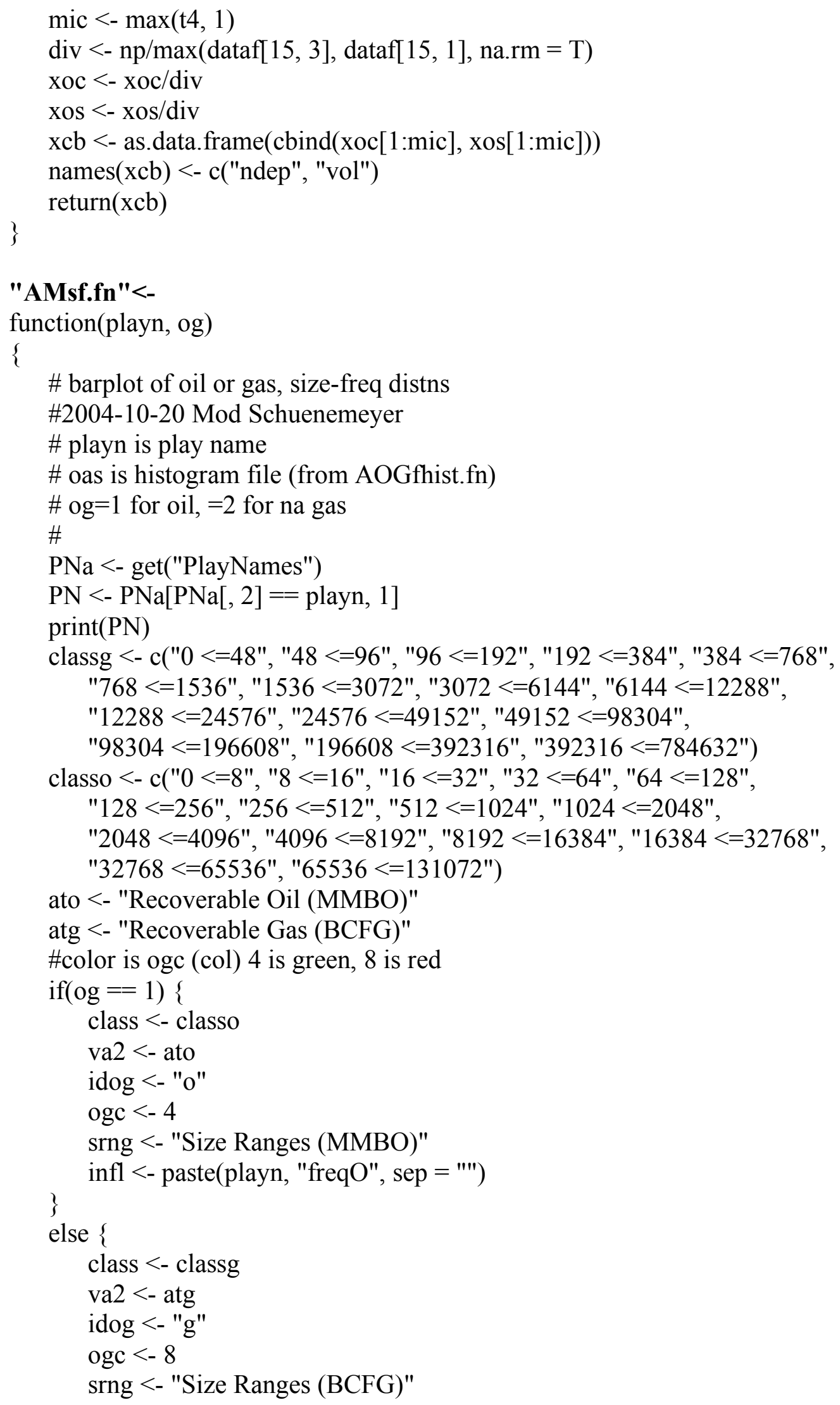




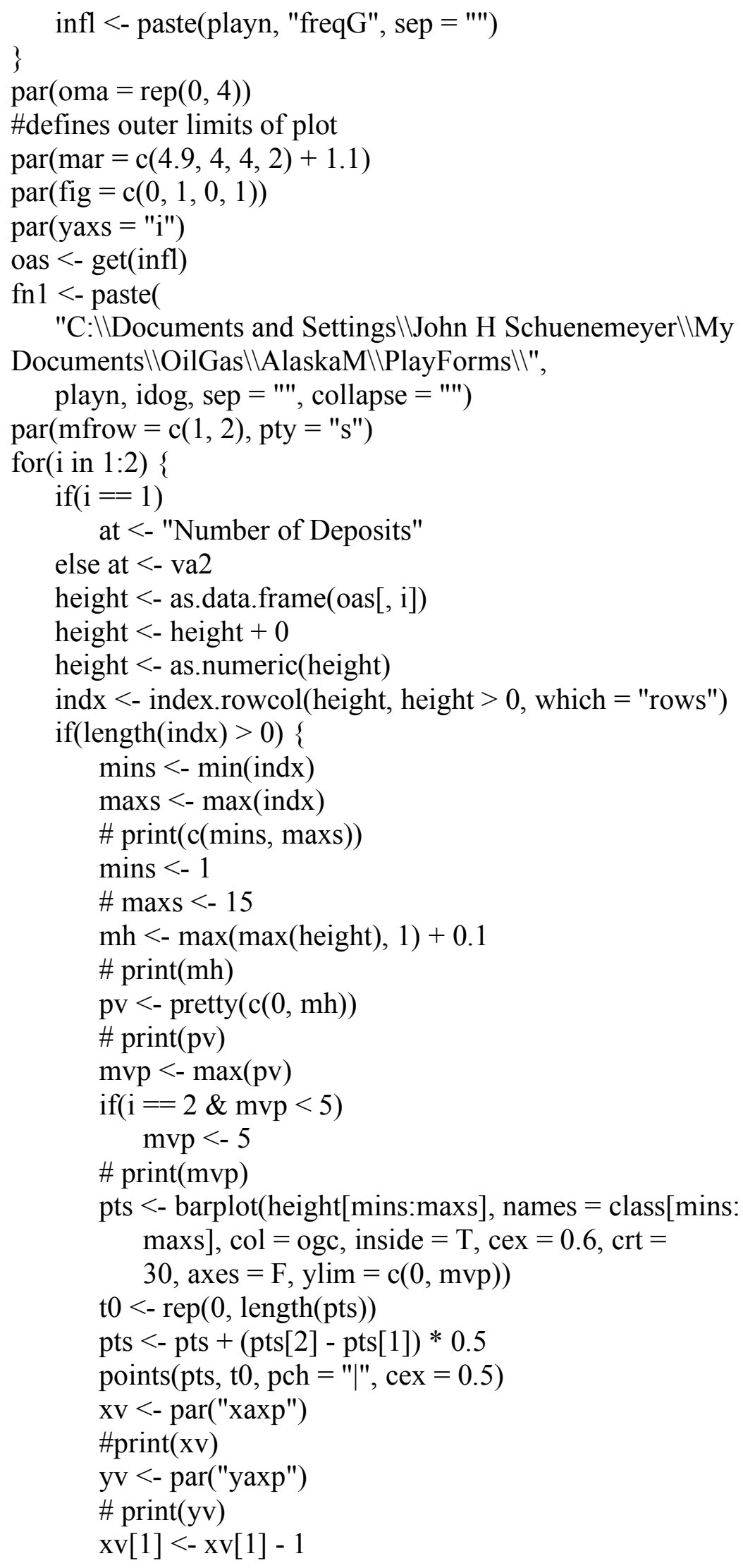




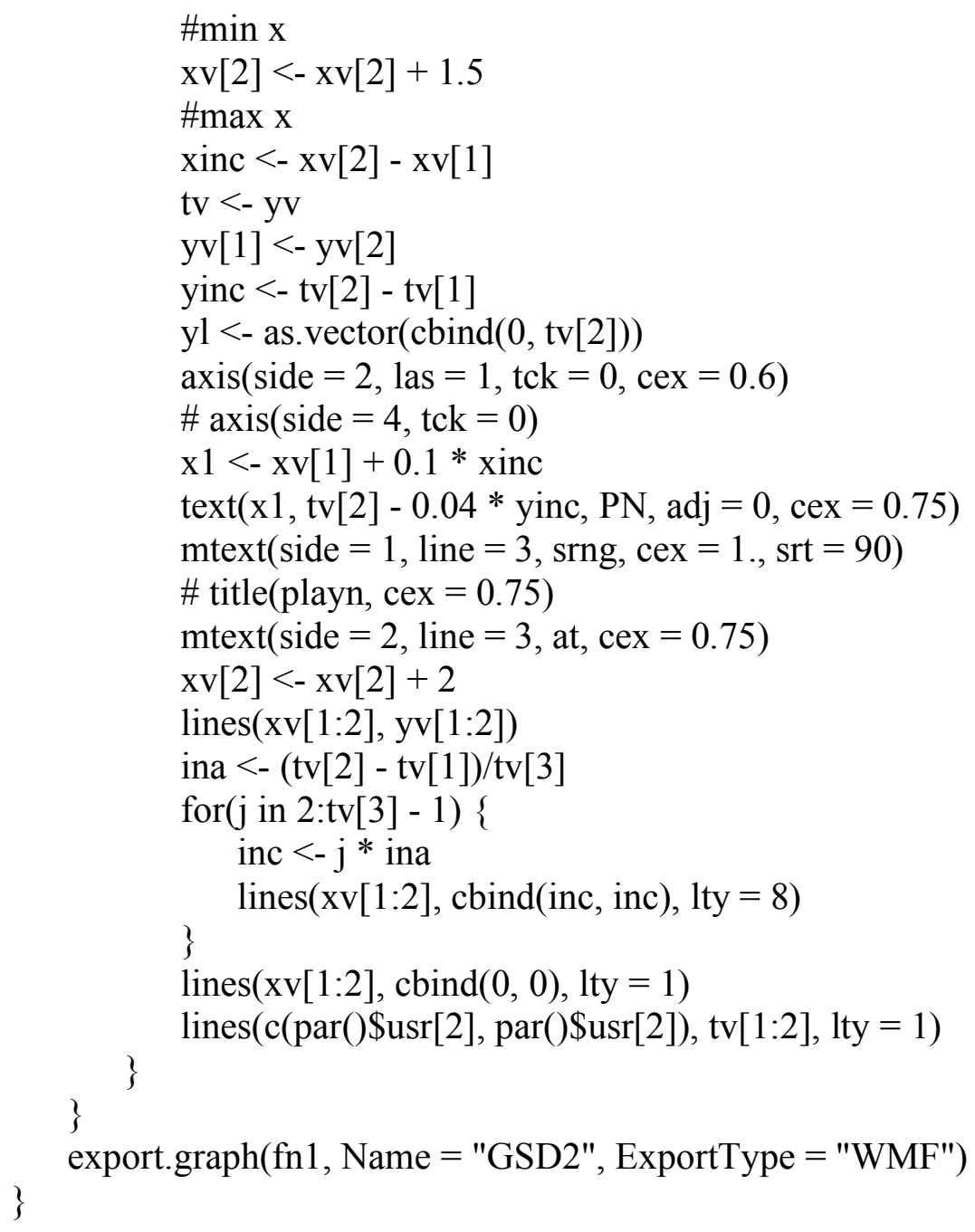




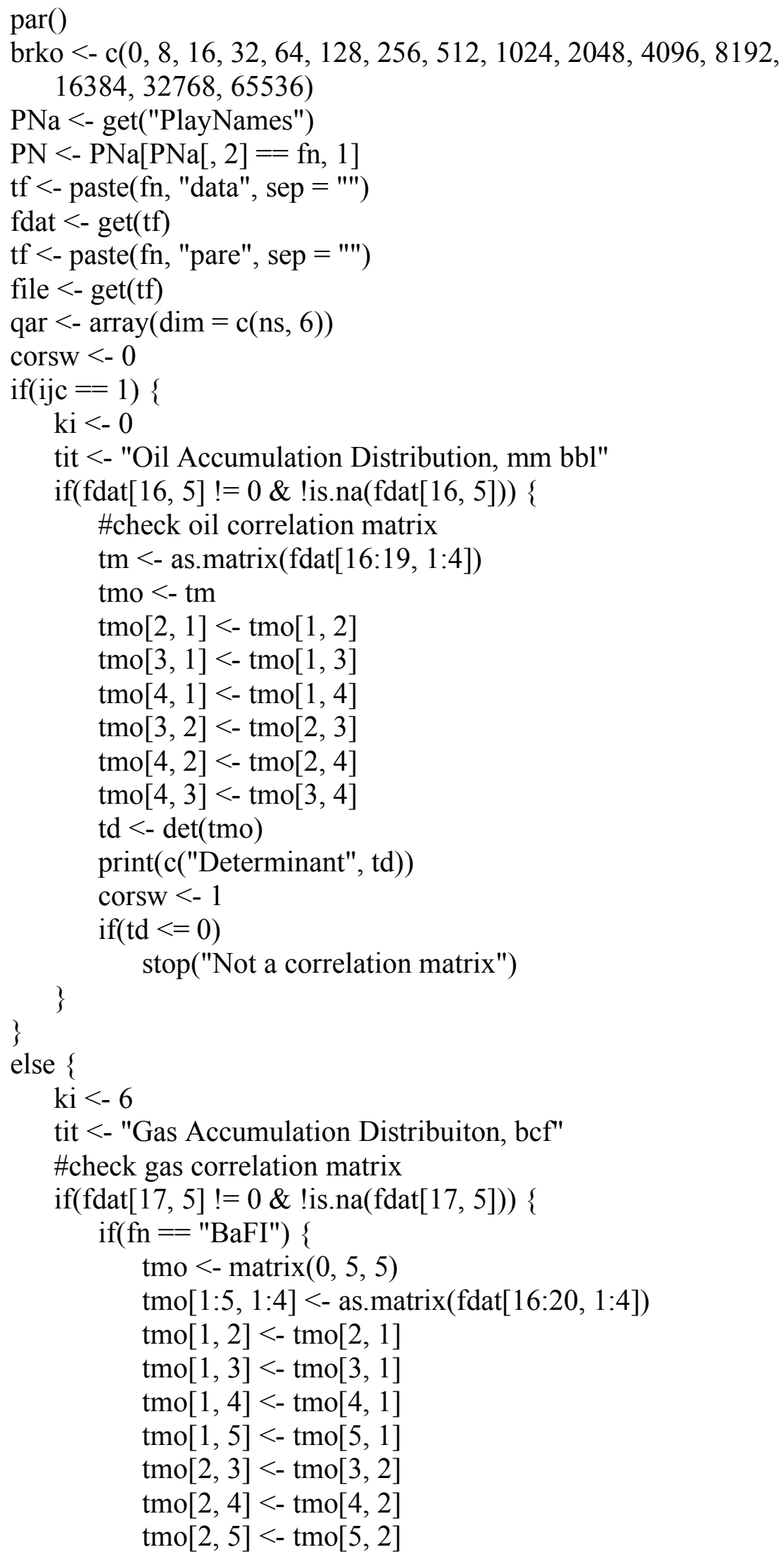




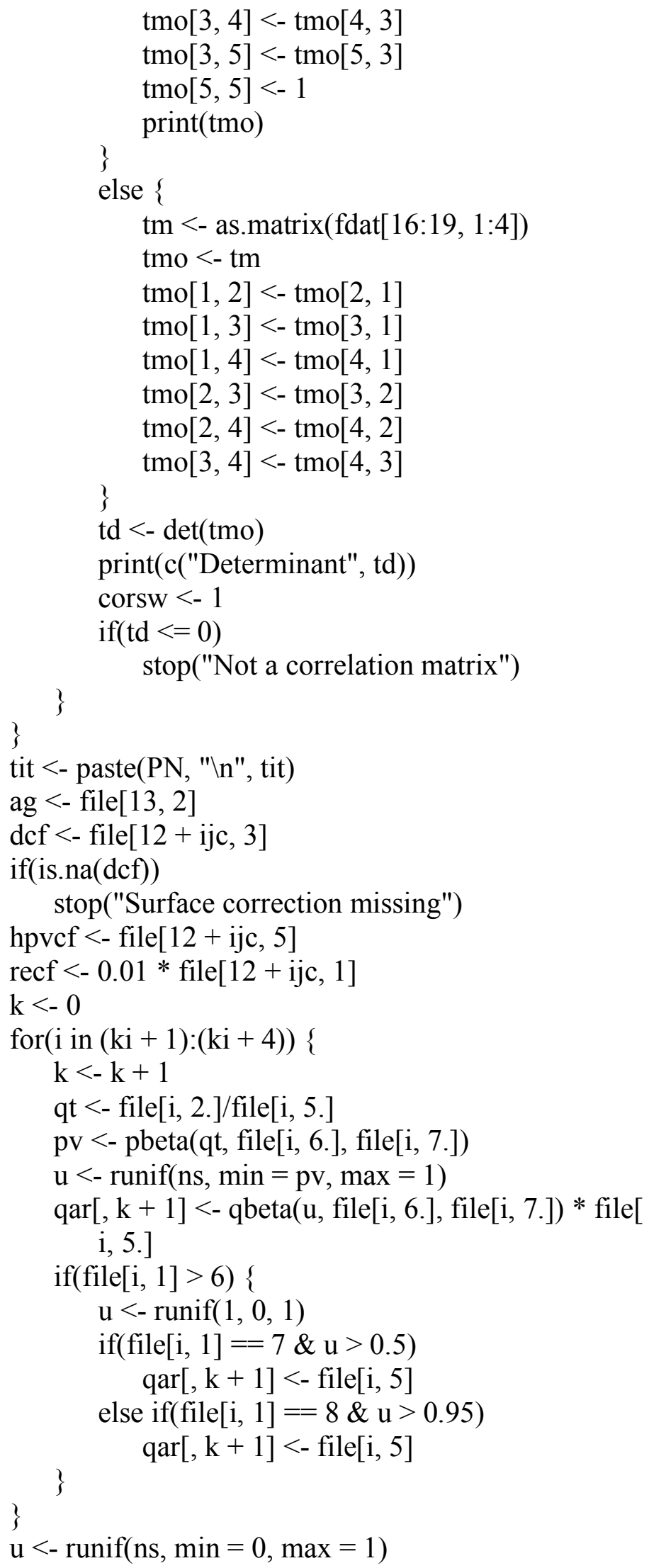




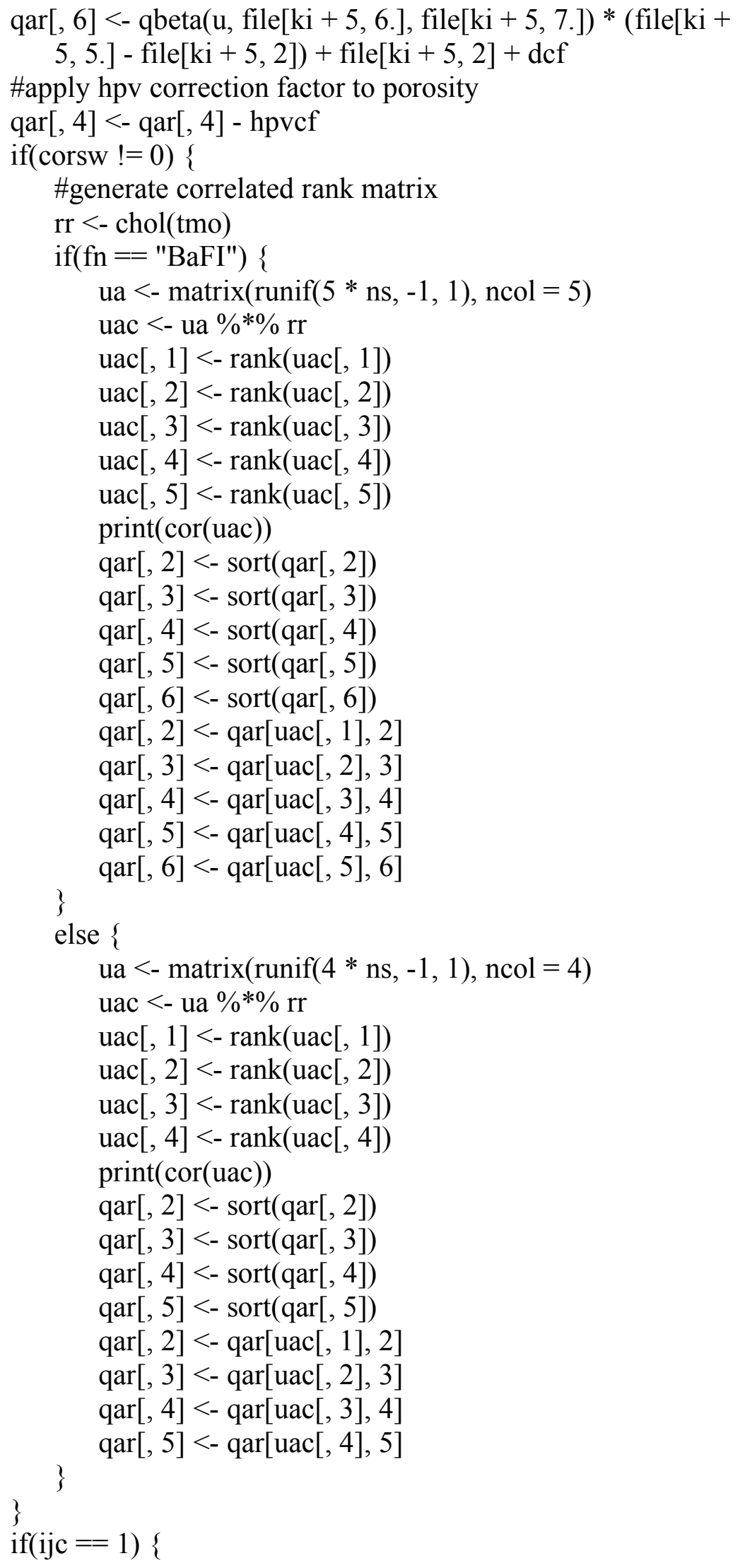




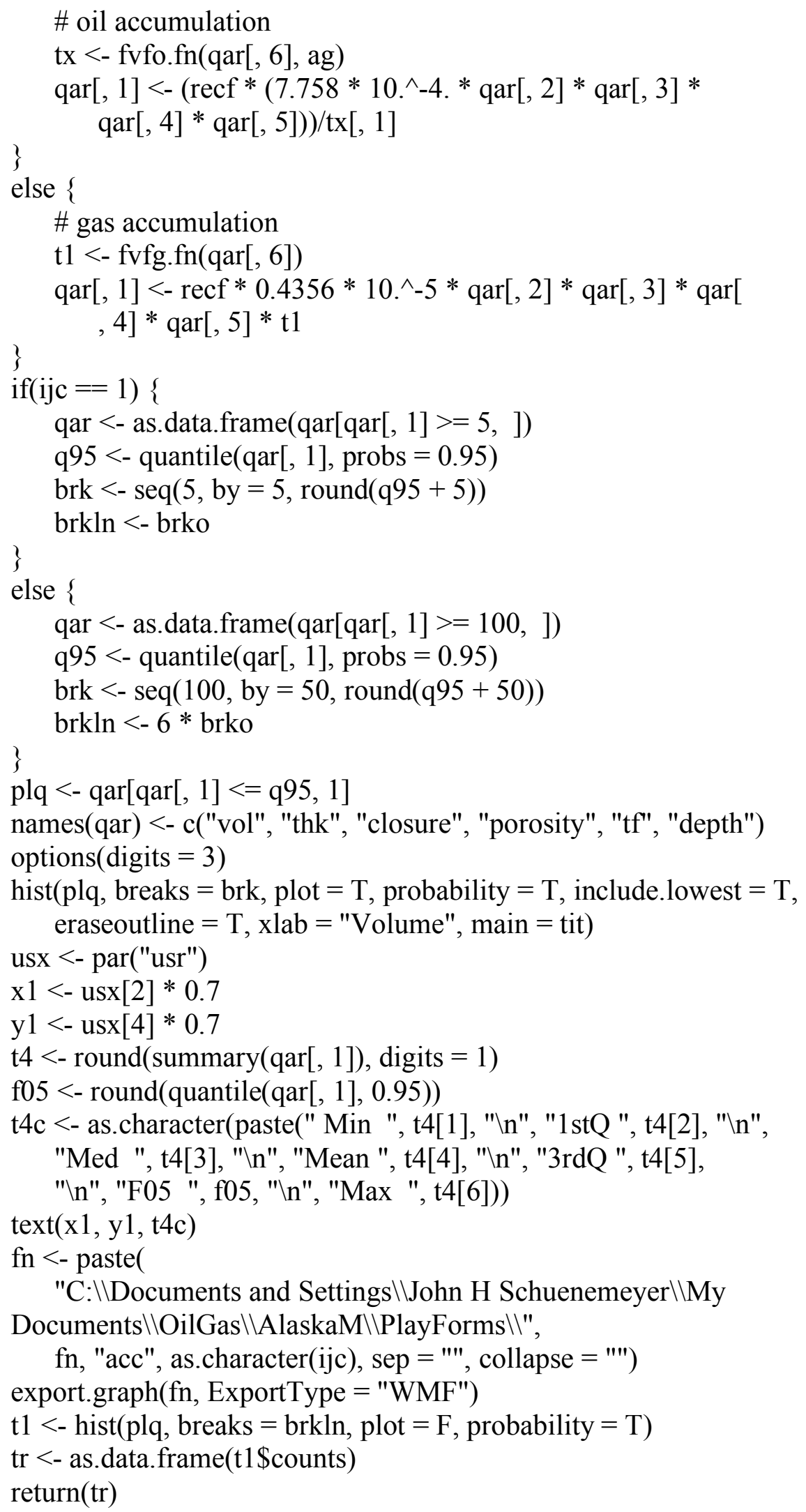




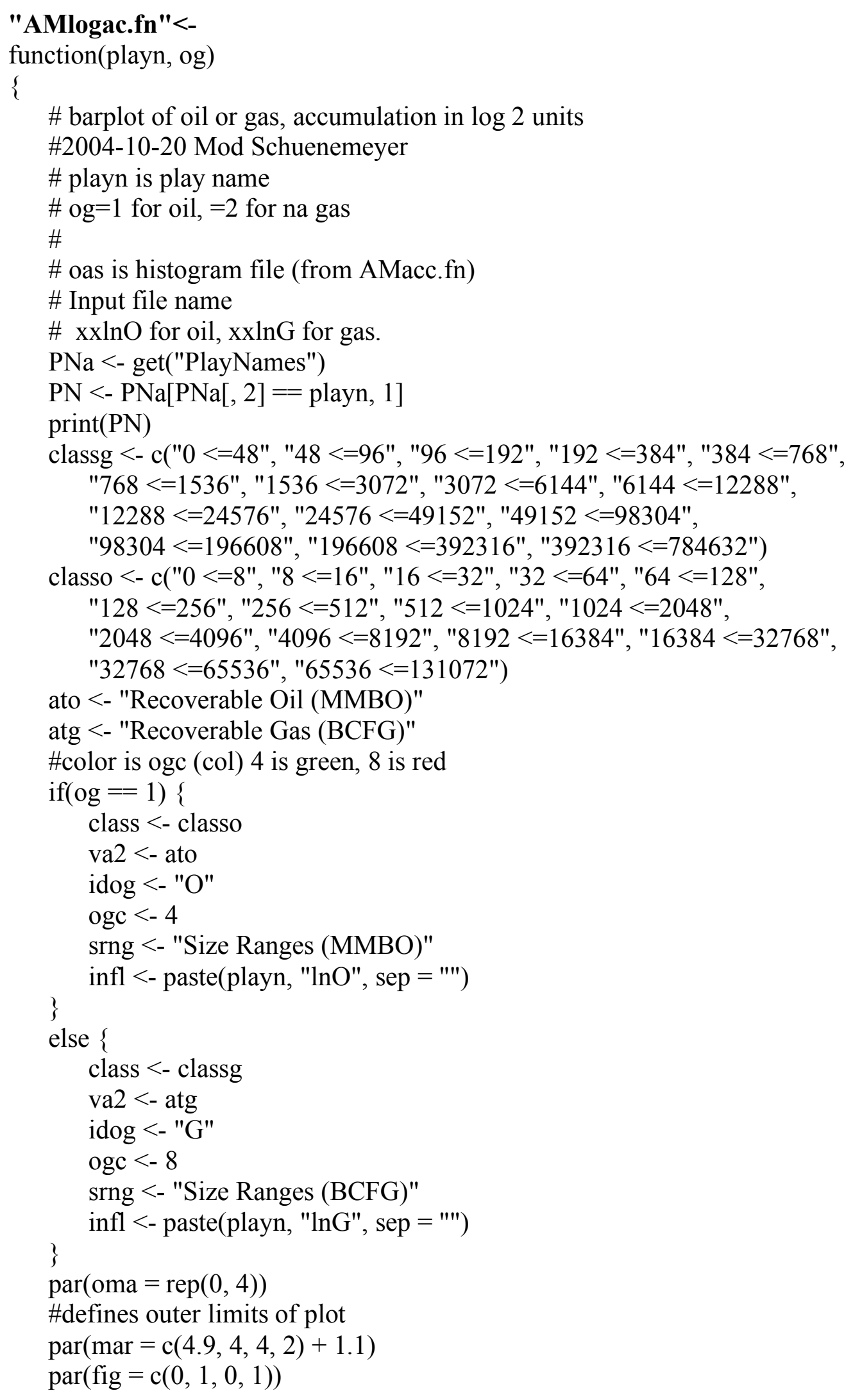




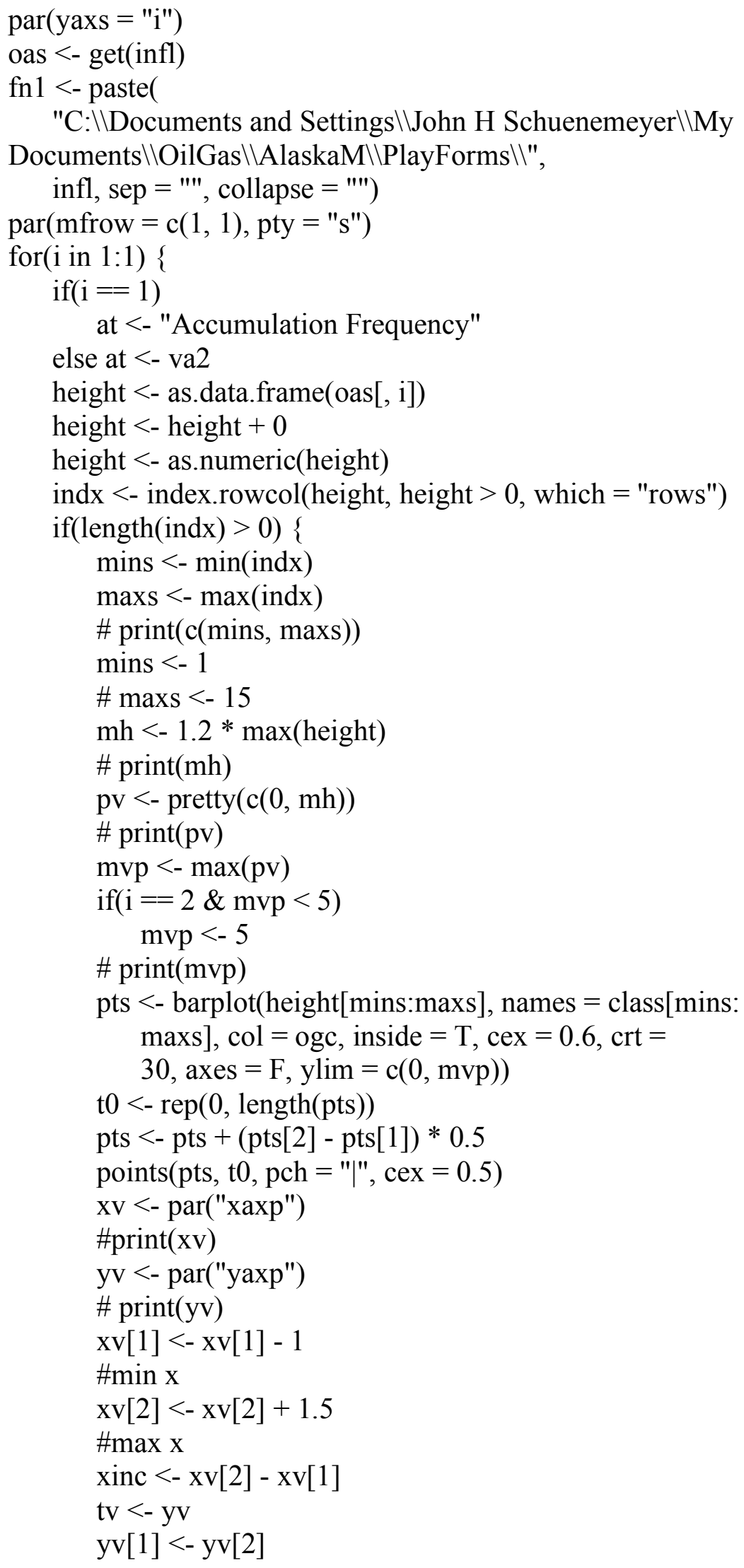




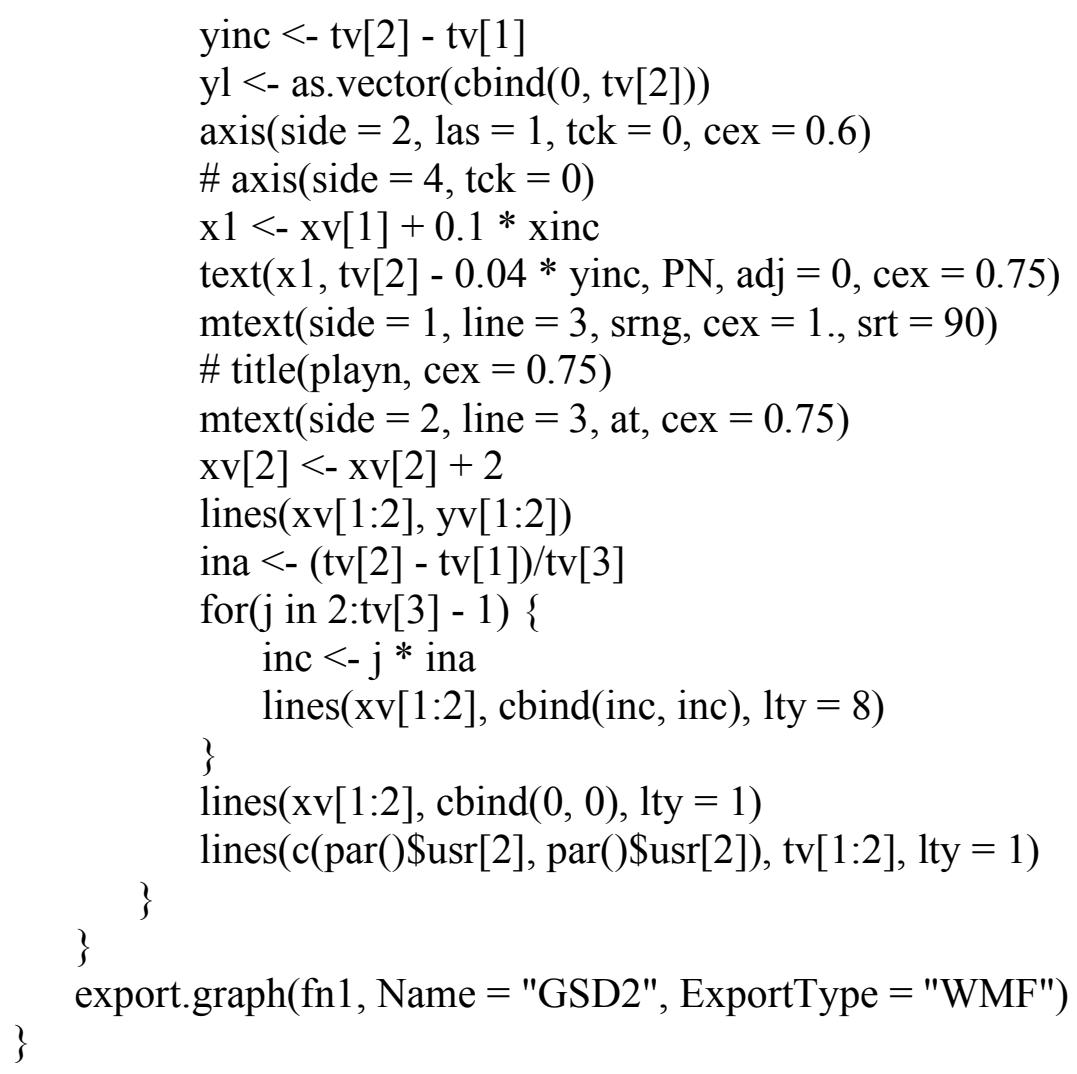




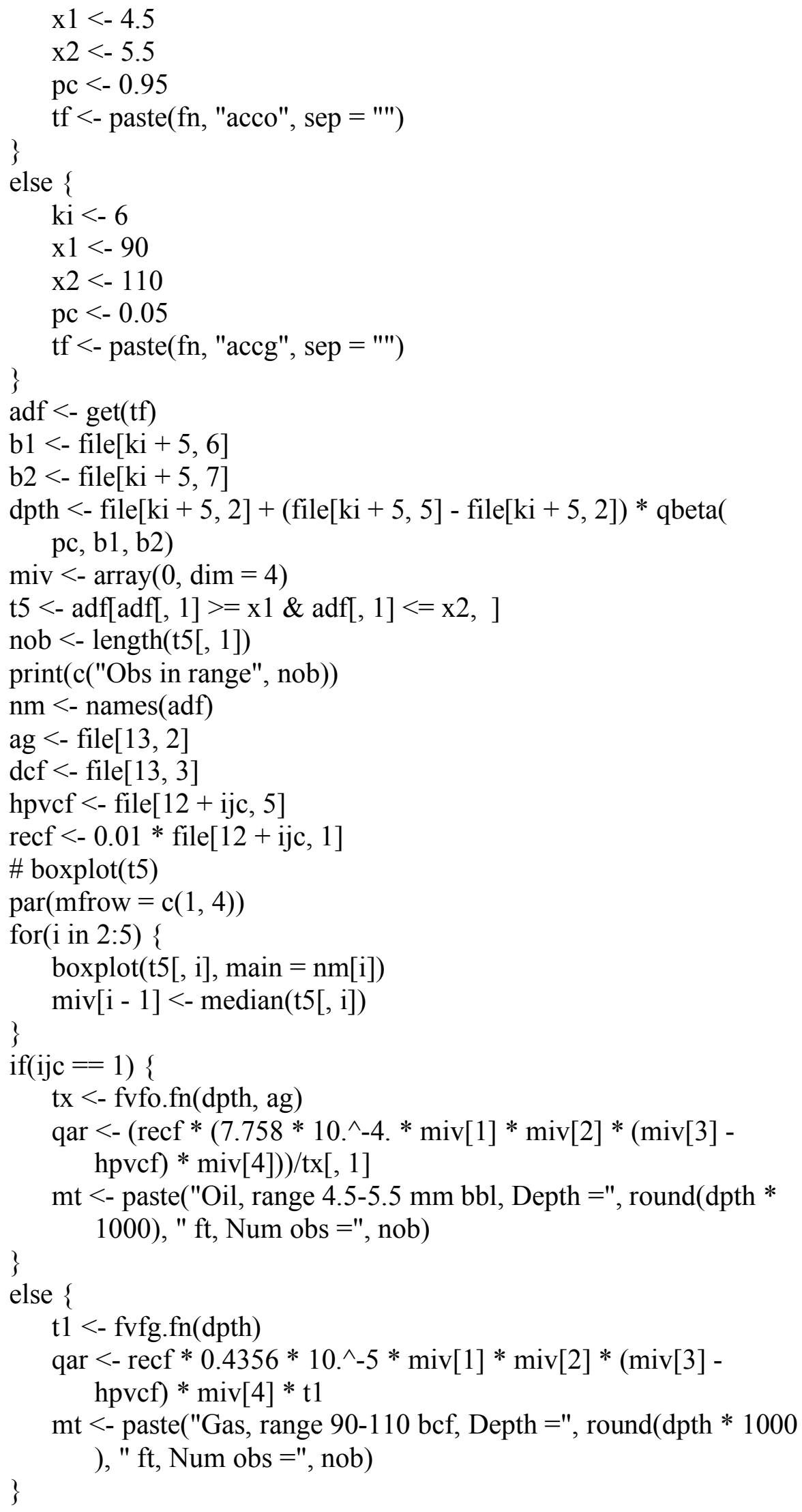



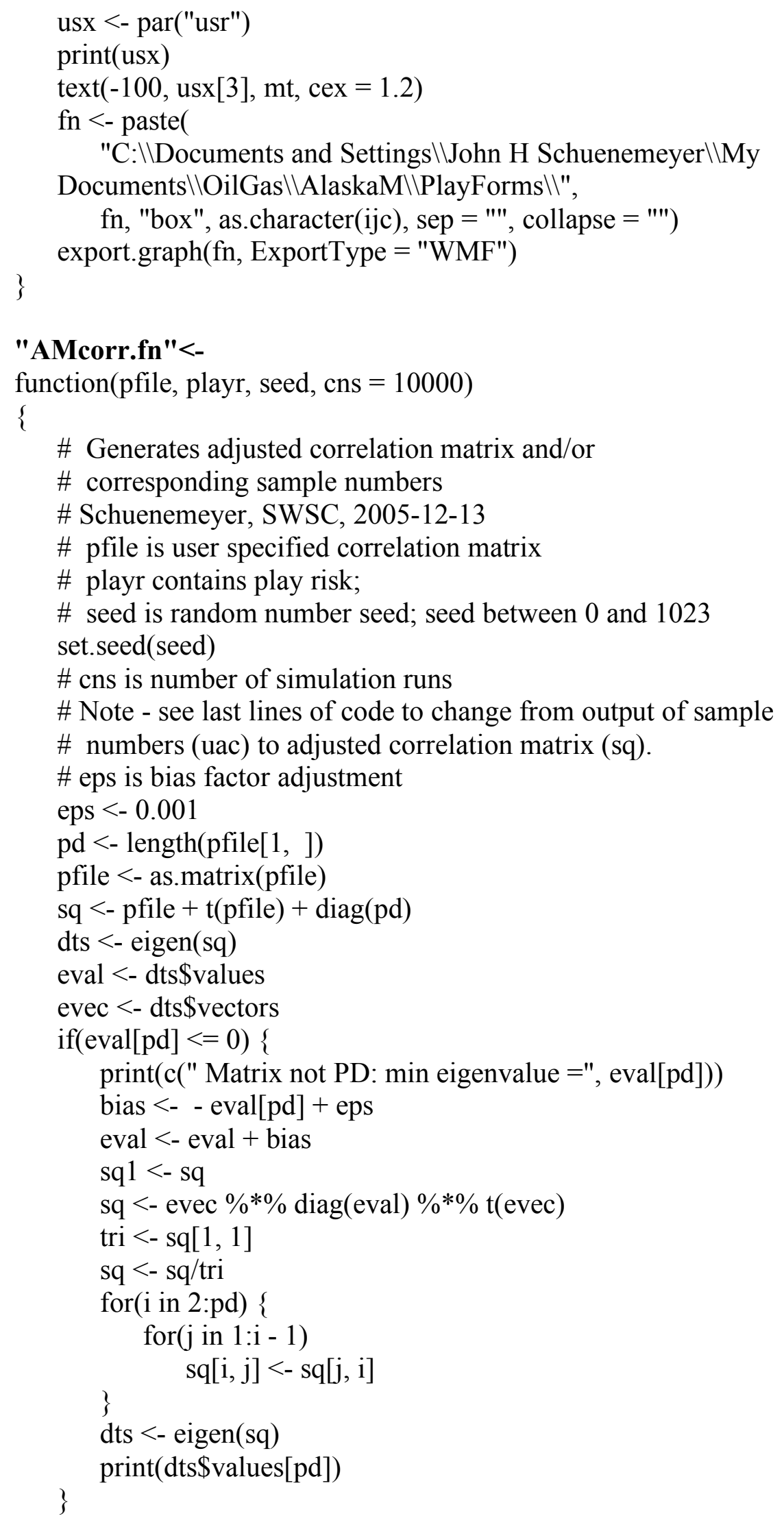


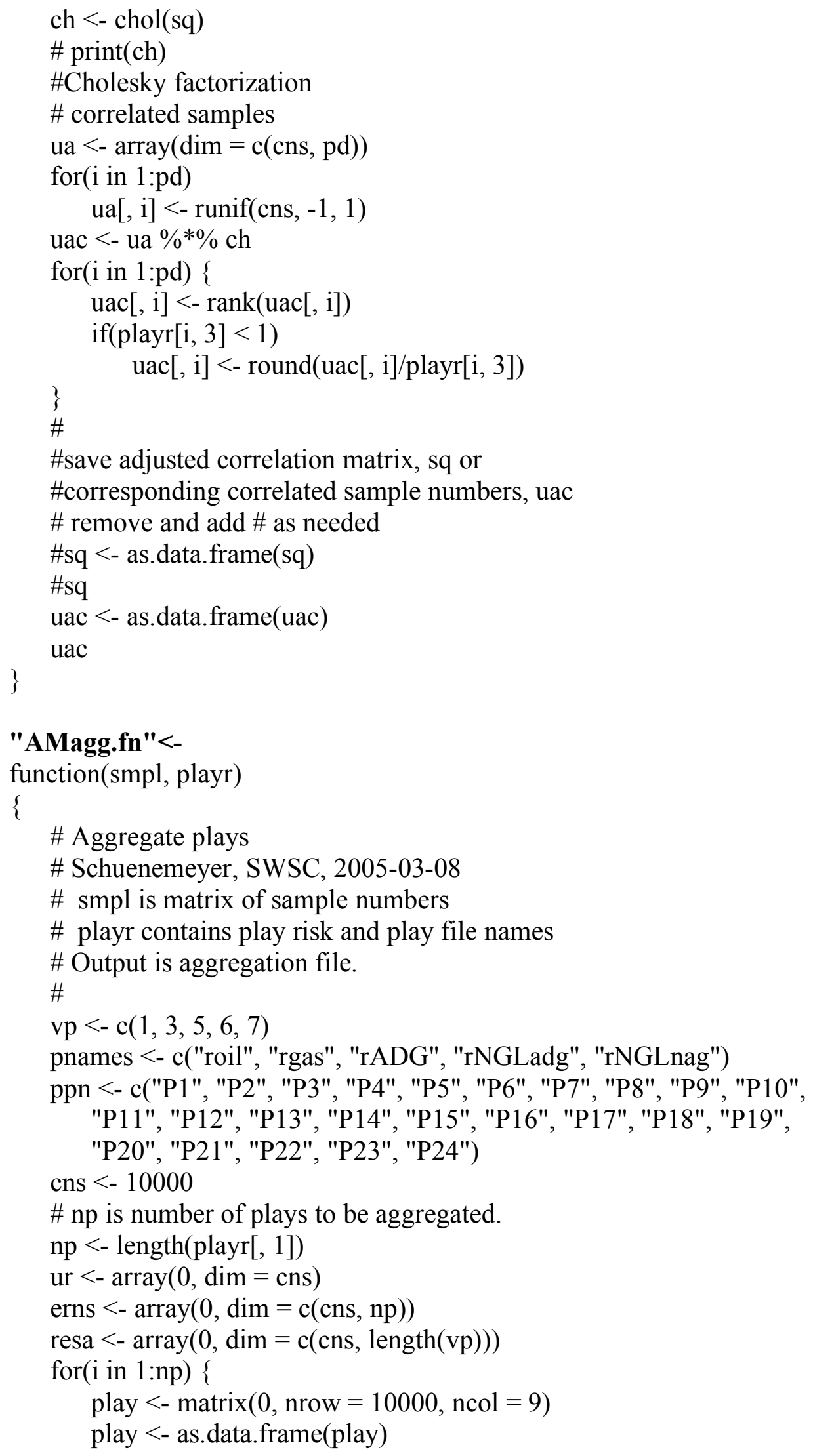




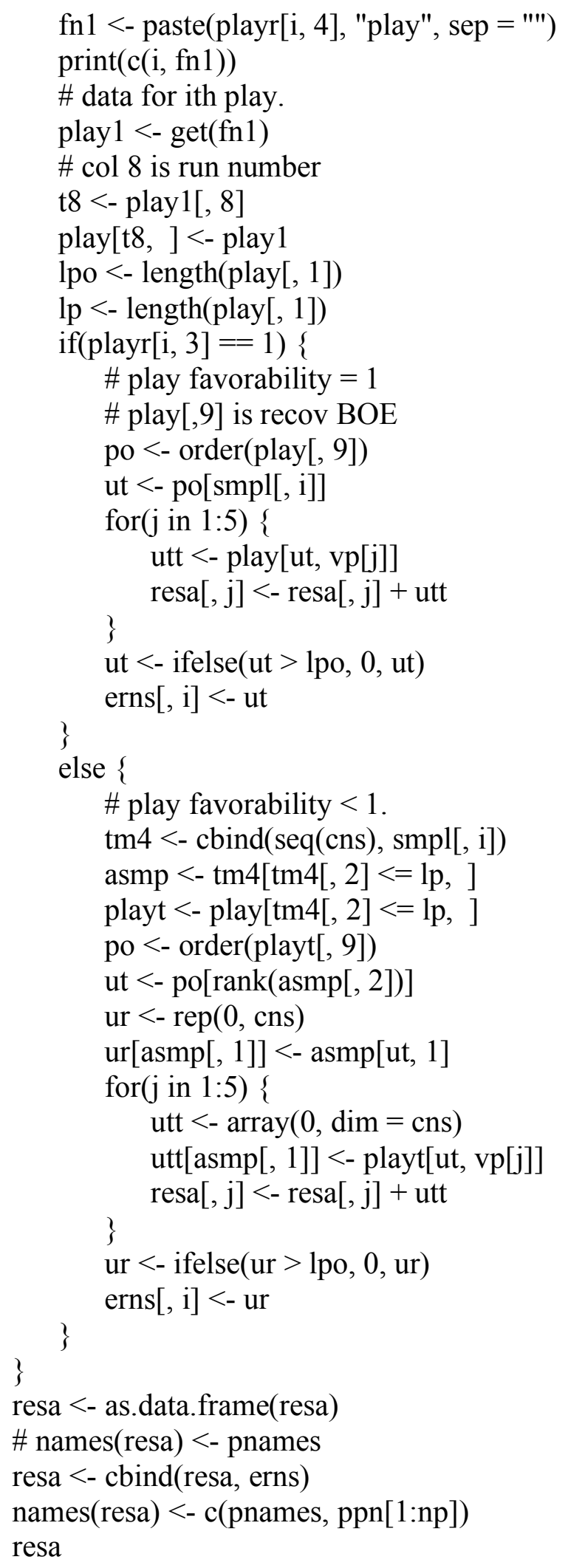




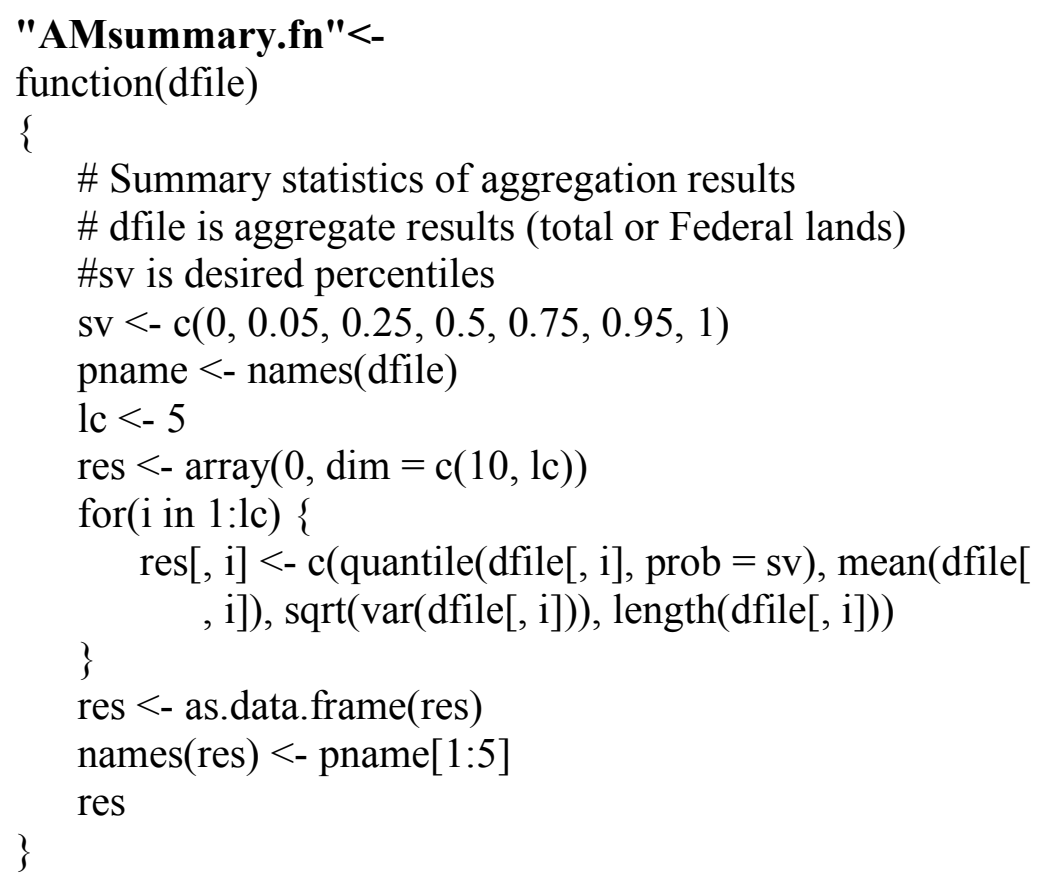

